\title{
Cosmological horizons and reconstruction of quantum field theories.
}

\author{
Claudio Dappiaggi ${ }^{1,3, a}$, Valter Moretti ${ }^{2,3, b}$, Nicola Pinamonti ${ }^{1,3, c}$ \\ ${ }^{1}$ II. Institut für Theoretische Physik, Universität Hamburg, Luruper Chaussee 149, D-22761 Hamburg, Germany. \\ ${ }^{2}$ Dipartimento di Matematica, Università di Trento and Istituto Nazionale di Fisica Nucleare - Gruppo Collegato \\ di Trento, via Sommarive 14 I-38050 Povo (TN), Italy. \\ ${ }^{3}$ Istituto Nazionale di Alta Matematica "F.Severi"- GNFM \\ E-mail: ${ }^{a}$ claudio.dappiaggi@desy.de, ${ }^{b}$ moretti@science.unitn.it, ${ }^{c}$ nicola.pinamonti@desy.de
}

Dedicated to Professor Klaus Fredenhagen on the occasion of his 60th birthday.

\begin{abstract}
As a starting point for this manuscript, we remark how the cosmological horizon of a certain class of Friedmann-Robertson-Walker backgrounds shares some non trivial geometric properties with null infinity in an asymptotically flat spacetime. Such a feature is generalized to a larger class of expanding spacetimes $M$ admitting a geodesically complete cosmological horizon $\Im^{-}$common to all co-moving observers. This property is later exploited in order to recast, in a cosmological background, some recent results for a linear scalar quantum field theory in spacetimes asymptotically flat at null infinity. Under suitable hypotheses on $M$ - valid for deSitter spacetime and some other FRW spacetimes obtained by perturbing deSitter space - the algebra of observables for a Klein-Gordon field is mapped into a subalgebra of the algebra of observables $\mathcal{W}\left(\Im^{-}\right)$constructed on the cosmological horizon. There is exactly one pure quasifree state $\lambda$ on $\mathcal{W}\left(\Im^{-}\right)$which fulfills a suitable energypositivity condition with respect to a generator related with the cosmological time displacements. Furthermore $\lambda$ induces a preferred physically meaningful quantum state $\lambda_{M}$ for the quantum theory in the bulk. If $M$ admits a timelike Killing generator preserving $\Im^{-}$, then the associated self-adjoint generator in the GNS representation of $\lambda_{M}$ has positive spectrum (i.e. energy). Moreover $\lambda_{M}$ turns out to be invariant under every symmetry of the bulk metric which preserves the cosmological horizon. In the case of an expanding deSitter spacetime, $\lambda_{M}$ coincides with the Euclidean (Bunch-Davies) vacuum state, hence being Hadamard in this case. Remarks on the validity of the Hadamard property for $\lambda_{M}$ in more general spacetimes are presented.
\end{abstract}

\section{Contents}

1 Introduction 2

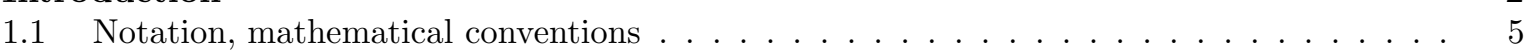

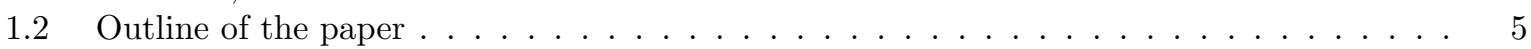

2 Cosmological horizons and asymptotically flatness 6

2.1 Friedmann-Robertson-Walker spacetime and cosmological horizons . . . . . . . . . . 6

2.2 Asymptotically-flat spacetime structure . . . . . . . . . . . . . . . . 7

2.3 FRW metrics with $\kappa=0$ and associated asymptotically flat structure . . . . . . . . . 9

2.4 Some examples . . . . . . . . . . . . . . . . . . . . 12 
3 General expanding universes with cosmological horizon and the horizon group. 13

3.1 Expanding universes with cosmological horizon $\Im^{-} \ldots \ldots \ldots \ldots \ldots$

3.2 The horizon symmetry group $G_{\Im^{-}} \ldots \ldots \ldots \ldots \ldots \ldots \ldots \ldots \ldots$

4 Linear Quantum Fields and preferred states induced by the cosmological horizon. 18

4.1 QFT in the bulk ........................ 18

4.2 Bosonic QFT on $\Im^{-}$and $G_{\Im^{-}}$-invariant states $\ldots \ldots \ldots \ldots \ldots \ldots \ldots$

$4.3 G_{\Im^{-}}$-invariant algebraic states on $\mathcal{W}\left(\Im^{-}\right) \ldots \ldots \ldots \ldots \ldots \ldots \ldots \ldots$

4.4 Interplay of QFT in $M$ and QFT on $\Im^{-} \ldots \ldots \ldots \ldots \ldots \ldots \ldots \ldots$

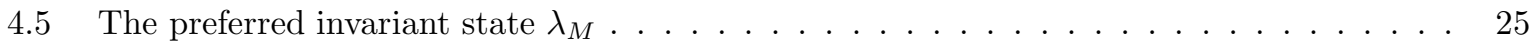

4.6 Testing the construction for the de Sitter case and for other FRW metrics . . . . . . . 27

5 Conclusions and open issues.

A Proof of some technical results.

B Quasifree states on Weyl algebras

\section{Introduction}

In the framework of quantum field theory over curved backgrounds we witnessed, in the past few year, an increased display of new and important formal results. In many cases we can track their origin in the existence of a non trivial interplay between some field theories living on a Lorentzian background - say $M$ - and a suitable counterpart constructed over a co-dimension one submanifold of $M$, often chosen as the conformal boundary of the spacetime. Usually thought of as a realization of the so-called holographic principle, this research line provided its most remarkable results in the framework of (asymptotically) AdS backgrounds. As a matter of fact, concepts such as Maldacena's conjecture AGM00 - in a string framework - or Rehren's duality (see [DR02] and references therein) - in the algebraic quantum field theory setting - nowadays, are appearing almost ubiquitously in the theoretical high-energy physics literature.

More recently a similar philosophy has been also adopted to deal with a rather different scenario, namely asymptotically flat spacetimes, where it is future null infinity $-\Im^{+} \sim \mathbb{R} \times \mathbb{S}^{2}$, i.e. the conformal boundary - which plays the role of the above-mentioned co-dimension one submanifold. Summarising our present day understanding briefly, null infinity, besides being endowed with universal geometric properties regardless of the bulk spacetime, is mainly characterised by the existence of a special and highly non trivial infinite dimensional symmetry group, the so-called Bondi-Metzner-Sachs group (BMS). In a series of papers started from [DMP06] and including [Mo06, Mo07, it was focused in particular on the fact that each solution of the massless wave equation conformally coupled to gravity (with compactly supported initial data) can be projected into a smooth function over $\Im^{+}$, preserving the relevant symplectic forms. (In [Da07] the result was extended to massive fields in Minkowski spacetime.) Employing the symplectic approach, it was the building block to formulate a classical field theory intrinsically living on null infinity.

It is important to pinpoint that the found bulk-to-boundary correspondence does not hold at a classical level only. In particular, it was shown that, to a certain extent, we can recast the above interplay also in the quantum field theory setting. As a matter of fact, under the further hypothesis of global hyperbolicity of the bulk spacetime (see DMP06 for more details), the set of solutions for the bulk wave equation conformally coupled to gravity is a strongly non degenerate symplectic space with, hence, an associated Weyl $C^{*}$-algebra. Barring some technical assumptions on the geometric structure of the boundary at future timelike infinity (see [Mo06] for details), the latter algebra can be isometrically embedded as a 
$C^{*}$-subalgebra of the boundary counterpart. Notice that this last algebra, defined on $\Im^{+}$, is universal, in the sense that it does not depend on the particular asymptotically flat spacetime under consideration. It was proved in [DMP06] that there exists a BMS-invariant, quasifree, pure algebraic state of the algebra over $\Im^{+}$, say $\omega$. That state is universal as the algebra of the observables on $\Im^{+}$and the geometric structure of $\Im^{+}$equipped with the BMS group are. At the level of first quantization, the notion of particle associated with $\omega$ coincides with a BMS invariant scalar particle constructed by means of a readjustment to the (nonlocally compact) BMS case of Wigner-Mackey's procedure for the Poincaré group. $\omega$ enjoys some further, a priori unexpected, non trivial properties. In [Mo06], it was proved that $\omega$ is the unique state displaying a certain positive-energy requirements, with respect to the GNS generators associated to timelike asymptotic symmetries of the spacetime. As a further interesting development obtained when an asymptotically flat spacetime $M$ is fixed, it was established in [Mo07] that $\omega$ can be pulled back from $\Im^{+}$to a state $\omega^{\prime}$ defined on the algebra of observables in the bulk $M$. Remarkably, $\omega^{\prime}$ turns out to be Hadamard and, as well, invariant under the action of any isometry of the bulk metric. In particular, the self-adjoint generator of timelike Killing isometries (if any) has positive spectrum without zero-modes. This is an important stability requirement. Finally, as shown in [DMP06, in Minkowski spacetime $\omega^{\prime}$ coincides to the standard Minkowski vacuum state. Hence $\omega^{\prime}$ appears to provide a natural candidate for a preferred state for the bulk field theory regardless of the existence of specific isometries of the metric. The construction of $\omega^{\prime}$ [DMP06] and the proof of the Hadamard property [Mo07, also if they were obtained independently, share some overlapp with an analogous, but physically different, construction due to Hollands [Ho00, concerning the definition of local vacuum states near every point in every spacetime.

Although, on the bright side, one could safely claim that all these mentioned results are compelling, on the dark side, one must be honest to admit that, to the date, they fail to be applicable to any physical model which one could realistically conceive to experimentally probe in the foreseen future. As a matter of fact, to a certain extent, such comment alas holds true from a more general perspective: when one thinks of a quantum field theory over curved background, it is often meant, as an implied rationale, that one is referring to or dealing with a theoretical model which is hardly testable by means of present time experiments. Such attitude is daily changing since new experiments in cosmology opened the road to probe effects and phenomena which are foreseen by different models describing the early stages of the universe. The most notable, in between the many nowadays available examples, is inflation where, in most models, the pivotal role is played by a single scalar field living on an (almost) de Sitter background. Although many of these models are mainly (though not only) studied at a classical level, it is to a certain extent mandatory to look for a deep-rooted analysis of the full-fledged underlying quantum field theory in order to achieve a more firm understanding of the model under analysis.

To this avail, the first, but to a certain extent, not appealing chance is to perform a case-by-case analysis of the quantum structure of all the possible models nowadays available. In our opinion a more attractive possibility is to look for some mean allowing us to draw some general conclusions or to point out some universal feature (as the existence of a null infinity equipped with the BMS symmetry group), independently from the chosen model or from the chosen background. Taking into account this philosophy, a natural "first step" to undertake would be to try to implement the previously discussed bulk-to-boundary correspondence which appear to encode, almost per construction, all the criteria of universality we are seeking for. This idea cannot be directly put into practice employing the model of an asymptotically flat spacetime, since most of the present day cosmological scenario do not envisage an asymptotically flat (or an asymptotically anti-de Sitter background) as the underlying spacetime of the present or past days Universe. Nonetheless, a way to make profitable use of some of our achievements in asymptotically flat spacetimes does exist as is established in this paper. As a starting point one could start noticing that roughly all the Early-Universe models assume, at least implicitly, the Cosmological Principle which leads 
the underlying background to be endowed with the widely-used Friedmann-Robertson-Walker (FRW) metrics. A direct inspection of the geometric properties of these spacetimes points out that, in most of the relevant physical cases (such as de Sitter to quote just one example), it exists a natural submanifold which, at first glance, appears to be a good candidate to replace null infinity in the role of preferred co-dimension 1 hypersurface: the cosmological (particle or event) horizon as defined by Rindler [Ri06. More precisely, in this paper we shall consider the cosmological particle horizon $\Im^{-}$, in common with all the co-moving observers, in order to deal with expanding universes. The first and, at the same time, one of the main aims of this manuscript is indeed to prove that, at a geometrical level, the cosmological horizon $\Im^{-}$has more deep-rooted connections than expected with the structure of future null infinity under some technical restriction on the analytic form of the expanding factor in the FRW metric with flat spatial section. Hence it will turn out that the horizon has a universal structure provided that bulk lies in a suitable class of FRW backgrounds and, hence, it represents the natural setting where to stage a bulkto-boundary correspondence. As in the more common scenario of asymptotically flat spacetime, in this analysis, a pivotal role is played by the conformal factor, that is the expanding factor in the FRW metric. In Penrose language, the conformal factor is the mean which allows us to recast a physical spacetime as an open subset of an unphysical manifold. Nonetheless, from a certain perspective, this scenario appears to be richer than that of asymptotically flat spacetimes, since we must also include a new ingredient in the full analysis of the horizon. An expanding universe admits a preferred future-oriented timelike vector field $X$ defining the worldlines of co-moving observers, whose common expanding rest-frames are the 3 -surfaces orthogonal to $X$. In FRW metrics $X$ is a conformal Killing field which becomes tangent to the cosmological horizon and, in the class of FRW metrics we consider, it individuates complete null geodesics on $\Im^{-}$.

This extent will be generalized to expanding spacetimes $M$ equipped with as geodesically complete cosmological horizon $\Im^{-}$and a conformal Killing field $X$, generally different from FRW spacetimes. The leading role of $X$ in such a construction is strengthened by its intertwining relation with the conformal factor which is a primary condition to take into account if one wants to study what is the structure of the symmetry group of the horizon (actually a subgroup of the huge full isometry group of the horizon viewed as semi-Riemannian manifold). We also address such an issue in the manuscript and, rather surprisingly, we discover that the situation is rather different from that of an asymptotically flat spacetime. As a matter of fact, the BMS is replaced by a new infinite dimensional group $S G_{\Im^{-}}$which has the structure of an iterated semidirect product i.e. it is $S O(3) \ltimes\left(C^{\infty}\left(\mathbb{S}^{2}\right) \ltimes C^{\infty}\left(\mathbb{S}^{2}\right)\right)$ where $S O(3)$ is the special orthogonal group with a three dimensional algebra, whereas $C^{\infty}\left(\mathbb{S}^{2}\right)$ stands for the set of smooth functions over $\mathbb{S}^{2}$ thought as an Abelian group under addition. The geometric meaning of $S G_{\Im^{-}}$is related with the following result. The subgroup of isometries of the spacetime which preserve the cosmological horizon structure is injectively mapped to a subgroup of $S G_{\Im^{-}}$. So that $S G_{\Im^{-}}$encodes some of the possible symmetries of the spacetime. However it must be remarked that $S G_{\Im^{-}}$is universal in the sense that it does not depend on the particular spacetime $M$ in the class under consideration.

Therefore the cosmological horizon appears to really be endowed with all the properties to be a natural candidate where to encode bulk data. Hence our aim is to show that, although different in several aspects from null infinity, it is still possible to trade in this cosmological scenario and, hence, to exploit many of the known results in the reconstruction of a bulk classical and quantum field.

Within this respect, we adopt a more generic starting point than that in DMP06, since we consider the set of solutions (with compactly supported initial data) of the Klein-Gordon equation of motion with both an arbitrary mass and coupling to curvature. As a result we find that, under suitable hypotheses on $M$ - valid, in particular, for certain FRW spacetimes which are de Sitter asymptotically - the algebra of observables $\mathcal{W}(M)$ of Klein-Gordon field in $M$ is one-to-one (isometrically) mapped to a subalgebra of the algebra of observables $\mathcal{W}\left(\Im^{-}\right)$naturally constructed on the cosmological horizon. In this sense 
information of quantum theory in the bulk $M$ is encoded by the quantum theory defined on the boundary $\Im^{-}$. Similarly to what found in the asymptotically flat space, it turns out that there is exactly one pure quasifree state $\lambda$ on $\mathcal{W}\left(\Im^{-}\right)$which fulfils a certain energy-positivity condition with respect to some generators of $S G_{\Im^{-}}$. The relevant generators are here those which can be interpreted as limit values on $\Im^{-}$of timelike Killing vectors of $M$, whenever one fixes a spacetime $M$ admitting $\Im^{-}$as the cosmological horizon. However, exactly as the geometric structure of $\Im^{-}, \lambda$ is universal in the sense that it does not depend on the particular spacetime $M$ in the class under consideration. The GNS-Fock representation of $\lambda$ individuates a unitary irreducible representation of $S G_{\Im^{-}}$. Fixing an expanding spacetime $M$ with complete cosmological horizon, $\lambda$ induces a preferred quantum state $\lambda_{M}$ for the quantum theory in $M$. $\lambda_{M}$ enjoys remarkable properties. It turns out to be invariant under all those isometries of $M$ (if any) that preserve the cosmological horizon structure. If $M$ admits a timelike Killing generator preserving $\Im^{-}$, the associated self-adjoint generator in the GNS representation of $\lambda_{M}$ has positive spectrum (i.e. energy). Finally, if $M$ is the very expanding de Sitter spacetime, $\lambda_{M}$ coincides to the Euclidean (Bunch-Davies) vacuum state, so that it is Hadamard in that case at least. Actually, Hadamard property seems to be valid in general, but that issue will be investigated elsewhere.

1.1. Notation, mathematical conventions. Throughout $\mathbb{R}^{+}:=[0,+\infty), \mathbb{N}:=\{0,1,2, \ldots\}$. For smooth manifolds $M, N, C^{\infty}(M ; N)$ (omitting $N$ whenever $N=\mathbb{R}$ ) is the space of smooth functions $f: M \rightarrow N . C_{0}^{\infty}(M ; N) \subset C^{\infty}(M ; N)$ is the subspace of compactly-supported functions. If $\chi: M \rightarrow N$ is a diffeomorphism, $\chi^{*}$ is the natural extension to tensor bundles (counter-, co-variant and mixed) from $M$ to $N$ (Appendix C in Wa84]). A spacetime $(M, g)$ is a Hausdorff, second-countable, smooth, fourdimensional connected manifold $M$, whose smooth metric has signature -+++ . We shall also assume that a spacetime is oriented and time oriented. We adopt definitions of causal structures of Chap. 8 in Wa84. If $S \subset M \cap \widehat{M},(M, g)$ and $(\widehat{M}, \widehat{g})$ being spacetimes, $J^{ \pm}(S ; M)\left(I^{ \pm}(S ; M)\right)$ and $J^{ \pm}(S ; \widehat{M})\left(I^{ \pm}(S ; \widehat{M})\right)$ indicate the causal (chronological) sets associated to $S$ and respectively referred to the spacetime $M$ or $\widehat{M}$.

1.2. Outline of the paper. In section 2 we introduce and we discuss the geometric set-up of the backgrounds that we are going to take into account throughout this paper. In particular we recall the notion of asymptotically flat spacetime and we find under which analytic conditions on the expanding factor, a Friedmann-Robertson-Walker (FRW) spacetime can be smoothly extended to a larger spacetime which is a past conformal completion of an asymptotically flat spacetime. In section 3 we provide a general definition for an expanding universe with a geodesically complete cosmological particle horizon and we analyse the implications of such a definition at a geometrical level. Furthermore we introduce and we discuss the structure of the horizon symmetry group showing its interplay with the possible isometries of the bulk metric. In section 4 we switch to a field theoretical perspective studying in 4.1 the structure of bulk scalar QFT and of the associated Weyl algebra, whereas, in 4.2, we deal with the horizon counterpart. In subsection 4.3, instead we analyse the structure of the algebraic states associated to the horizon algebra emphasising in particular the existence of a preferred state invariant under the full symmetry group, which enjoys some uniqueness/energy-positivity properties. Subsections 4.4 and 4.5 are devoted to the development of the interplay between the bulk and the boundary theory; a particular emphasis is given to the selection of a natural preferred bulk states and on the analysis of its properties. Since all these conclusions are based upon some a priori assumptions on the behaviour of the solutions in the bulk of the Klein-Gordon equation with a generic coupling to curvature, we shall devote section 4.6 to test these requirements. Eventually, in section 5, we draw some conclusions and we provide some hints on future research perspectives. 


\section{Cosmological horizons and asymptotically flatness}

2.1. Friedmann-Robertson-Walker spacetime and cosmological horizons. A homogeneous and isotropic universe can be locally described by a smooth spacetime, in the following indicated by $\left(M, g_{F R W}\right)$, where $M$ is a smooth Lorentzian manifold equipped with the following Friedmann-Robertson-Walker (FRW) metric

$$
g_{F R W}=-d t \otimes d t+a(t)^{2}\left[\frac{1}{1-\kappa r^{2}} d r \otimes d r+r^{2} d \mathbb{S}^{2}(\theta, \varphi)\right]
$$

Above, $d \mathbb{S}^{2}(\theta, \varphi)=d \theta \otimes d \theta+\sin ^{2} \theta d \phi \otimes d \phi$ is the standard metric on the unit 2-sphere and, up to normalisation, $\kappa$ can take the values $-1,0,1$ corresponding respectively to an hyperbolic, flat and closed spacetimes. The coordinate $t$ ranges in some open interval $I$. Here $a(t)$ is a smooth function of $t$ with constant sign (since $g$ is nondegenerate). Henceforth we shall assume that $a(t)>0$ when $t \in I$. We also suppose that the field $\partial_{t}$ individuates the time orientation of the spacetime.

Physically speaking and in the universe observed nowadays, the sections of $M$ at fixed $t$ are the isotropous and homogeneous 3 -spaces containing the matter of the universe, the world lines describing the histories of those particles of matter being integral curves of $\partial_{t}$. In this picture, the cosmic time $t$ is the propertime measured at rest with each of these cluster, whereas the scale $a(t)$ measures the size of the observed cosmic expansion in function of $t$.

The metric (11) may enjoy two physically important features. Consider a co-moving observer pictured by a integral line $\gamma=\gamma(t), t \in I$, of the field $\partial_{t}$ and focus on $J^{-}(\gamma)$. If $J^{-}(\gamma)$ does not cover the whole spacetime $M$, the observer $\gamma$ cannot receive physical information from some events of $M$ during his/her story: Causal future-directed signals starting from $M \backslash J^{-}(\gamma)$ cannot achieve any point on $\gamma$. In other words, and adopting the terminology of Ri06, a cosmological event horizon takes place for $\gamma$. It is the null 3-hypersurface $\partial J^{-}(\gamma)$. Conversely, whenever $J^{+}(\gamma)$ does not cover the whole spacetime $M$, physical information sent by the observer $\gamma$ during his/her story is prevented from getting to some events of $M$ : Causal future-directed signals starting from $\gamma$ do not reach any point in $M \backslash J^{+}(\gamma)$. In this case, exploiting again the terminology of [Ri06], a cosmological particle horizon exists for $\gamma$. It is the null 3-hypersurface $\partial J^{+}(\gamma)$.

As is well-known, a sufficient condition for the appearance of cosmological horizons can be obtained from the following analysis. One re-arranges the metric (1) into the form

$$
g_{F R W}=a^{2}(\tau)\left[-d \tau \otimes d \tau+\frac{1}{1-\kappa r^{2}} d r \otimes d r+r^{2} d \mathbb{S}^{2}(\theta, \varphi)\right],
$$

where

$$
\tau(t)=d+\int a^{-1}(t) d t
$$

is the conformal cosmological time, $d \in \mathbb{R}$ being any fixed constant. By construction $\tau=\tau(t)$ is a diffeomorphism from $I$ to some open, possibly infinite, interval $(\alpha, \beta) \ni \tau$. Notice that $\partial_{\tau}$ is a conformal Killing vector field whose integral lines coincide (up to the parametrisation) with the integral lines of $\partial_{t}$. Thus the time $\tau$ has a natural geometric meaning related with the FRW metric. Since the causal sets $J^{ \pm}$ are fixed under smooth positive rescaling of the metric, one may study the causal sets $J^{ \pm}(\gamma)$ referring to the ultrastatic metric

$$
g:=-d \tau \otimes d \tau+\frac{1}{1-\kappa r^{2}} d r \otimes d r+r^{2} d \mathbb{S}^{2}(\theta, \varphi)
$$


rather than the original one. Causal structures of this ultrastatic metric are much easier to study than those of $g_{F R W}$, due to the isometry group associated with the coordinate $\tau$. Referring to the metric $g$, the manifold $M$ turns out to be a product $(\alpha, \beta) \times \Sigma$ from the metrical point of view, too; where $\Sigma$ is the spatial section equipped with the constant-curvature metric

$$
\frac{1}{1-\kappa r^{2}} d r \otimes d r+r^{2} d \mathbb{S}^{2}(\theta, \varphi)
$$

As a byproduct, this entails that the spacetime $\left(M, g_{F R W}\right)$ is globally hyperbolic, because such properties holds for every ultrastatic spacetime and, furthermore, the causal structure of a spacetime is invariant under smooth positive conformal rescaling of the metric.)

A straightforward analysis based on the shape of the metric $g$ establishes that $J^{-}(\gamma)$ does not cover he whole spacetime $M$ whenever $\beta<+\infty$. In that case a cosmological event horizon takes place for $\gamma$. Similarly $J^{+}(\gamma)$ does not cover the whole spacetime $M$ whenever $\alpha>-\infty$. In that case a cosmological particle horizon takes place for $\gamma$. In both cases the horizons $\partial J^{-}(\gamma)$ and $\partial J^{+}(\gamma)$ are null 3-hypersurfaces diffeomorphic to $\mathbb{R} \times \mathbb{S}^{2}$, made of null geodesics of $g_{F R W}$. One may think of these surfaces as the limit light-cones emanating from $\gamma(t)$, respectively towards the past or towards the future, as $t$ tends to $\sup I$ or inf $I$ respectively. The tips of the cones generally get lost in the limit procedure: In realistic models $\alpha$ and $\beta$ correspond, when they are finite, to a big bang or a big crunch respectively. As a general comment, we stress that the cosmological horizons introduced above generally depend on the fixed observer $\gamma$.

Remark 2.1. The requirement on the finiteness of the bounds $\alpha$ and $\beta$ for the range of the conformal cosmological time $\tau$ are sufficient conditions for the existence of the cosmological horizons, but they are by no means necessary. Indeed it may happen that - and this is the case of de Sitter spacetime - there is, indeed a cosmological horizon arbitrarily close to $M$, but outside $M$. This happens when the spacetime $M$ and its metric can be extended beyond its original region $M$ to a larger spacetime $(\widehat{M}, \widehat{g})$ so that it happens $\partial M=\partial J^{ \pm}(M ; \widehat{M})$. The cosmological horizon $\Im^{ \pm}$coincides to the boundary $\partial M$ and, by construction, it does not depend on the considered observer $\gamma$ (an integral curve of the field $\partial_{t}$ ) evolving in $M$. Referring in particular to a conformally static region $M$ (equipped with the metric (11) for $\kappa=0$ ) embedded in the complete de Sitter spacetime $\widehat{M}, \partial M$ turns out to be a null surface with the topology of $\mathbb{R} \times \mathbb{S}^{2}$. In the following we shall focus on this type of cosmological horizons.

2.2. Asymptotically-flat spacetime structure. The cosmological horizons depicted in remark 2.1 give rise to an interesting interplay of Friedmann-Robertson-Walker spacetimes and asymptotically flat spacetimes at null infinity. In particular one may try to extend some important recent results [DMP06, Mo06, Mo06, Da07. concerning Quantum Field Theory, to QFT in Friedmann-Robertson-Walker spacetimes. This is, in fact, the main goal of this paper. We remind here some general definitions concerning asymptotically flat spacetime theory - and the notion of null boundaries at infinity $\Im^{ \pm}$in particular - which will be useful in the rest of the paper. The following, apparently complicated, definition captures the mathematical structure of those spacetimes which look like Minkowski spacetime as soon as one approaches the infinity along light rays. The null infinity $\Im^{-}$is viewed as the boundary of $M$ when it is embedded into a larger spacetime $(\widehat{M}, \widehat{g})$ by means of a conformal isometry.

Definition 2.1. A smooth four-dimensional spacetime $(M, g)$ is called asymptotically flat vacuum spacetime at future null infinity if it exists a second four dimensional spacetim $1 \widehat{1}(\widehat{M}, \widehat{g})$, a suitable

\footnotetext{
${ }^{1}$ This definition may be relaxed since the region $\widehat{M} \backslash\left(M \cup \Im^{+}\right)$plays no role in the original definitions by Geroch, Ashteker Ge77, AX78] and others (but it did in [DMP06, Mo06] Mo07]). Preserving the rest of the definition with $\widetilde{M}$ in place of $\widehat{M}$, the existence of the spacetime $(\widehat{M}, \widehat{g})$ may be weakened to the existence of a manifold with boundary $\widetilde{M}$,
} 
embedding $\lambda: M \rightarrow \lambda(M) \subset \widehat{M}$ (we shall omit to write explicitly it in the following for the sake of the simplicity), and a smooth function $\Omega: M \rightarrow(0,+\infty)$ such that $\widehat{g}=\Omega^{2} g$ and the following facts hold

1. Existence and causal/topological properties of null infinity. $M$ (through $\lambda$ ) is the interior of a submanifold with boundary of $\widehat{M}$, the boundary $\Im^{+}:=\partial M$ being diffeomorphic to $\mathbb{R} \times \mathbb{S}^{2}$ and $\Im^{+} \cap J^{-}(M ; \widehat{M})=\emptyset$.

2. Asymptotic flatness. $(M, g)$ is strongly causal and satisfies vacuum Einstein equations in a neighbourhood of $\Im^{+}$.

3. $\Omega-\Im^{+}$interplay. $\Omega$ extends to a smooth function on $\widehat{M}$ such that $\Omega \uparrow_{\Im^{+}}=0$ but $d \Omega \neq 0$ everywhere on $\Im^{+}$.

4. Geodesic completeness of $\Im^{+}$. Redefining $\Omega^{\prime}:=\omega \Omega$ and $\widehat{g}^{\prime}:=\omega^{2} \widehat{g}$ correspondingly, where $\omega>0$ is defined in a neighbourhood of $\Im^{+}$and satisfies $\widehat{\nabla}_{a}\left(\omega^{4} \widehat{g}^{a b} \widehat{\nabla}_{b} \Omega\right)=0$ on $\Im^{+}$, the integral lines of $\widehat{\nabla}^{\prime b} \Omega^{\prime}$ in $\Im^{+}$are complete.

The manifold $\Im^{+}$is called the future null infinity of $M$ and $(\widehat{M}, \widehat{g})$ is a future conformal completion of $(M, g)$. There is a completely analogous definition of asymptotically flat vacuum spacetime at past infinity replacing $J^{-}(M ; \widehat{M})$ with $J^{+}(M ; \widehat{M})$ as well as $\Im^{-}$with $\Im^{+}$.

Remark 2.2. (1) It is not self-evident from the given definition that $\Im^{+}$is a null surface and the complete curves tangent to $\widehat{\nabla}^{\prime b} \Omega^{\prime}$ are null-like geodesics as well. It is proved explicitly in the Appendix. (2) This observation will turn out to be relevant in the following. The metric on $\Im^{+}$is unphysical, since $\Im^{+}$is out of the universe, and, thus, it can be rearranged by varying the factor $\omega$. It is always possible to fix some of the remaining degrees of freedom of $\omega$ [Wa84, obtaining $\omega_{B}$ so that the metric $\omega_{B}^{2} \widehat{g}$ takes the Bondi form on $\Im^{+}$:

$$
\omega_{B}^{2} \widehat{g}\left\lceil\Im^{+}=-d \ell \otimes d \Omega_{B}-d \Omega_{B} \otimes d \ell+d \mathbb{S}^{2}(\theta, \varphi) .\right.
$$

where $\Omega_{B}:=\omega_{B} \Omega$ is used as a coordinate about $\Im^{+}$and $\ell \in \mathbb{R}$ is the affine paramete 2 of the complete null geodesics of $\omega_{B}^{2} \widehat{g}$ forming $\Im^{+}$. Above we have used coordinates $\ell, \Omega_{B}, \theta, \phi$ defined in a neighbourhood of $\Im^{+}$essentially as a consequence of $d \omega \Omega=\omega d \Omega \neq 0$ on $\Im^{+}$(see [Wa84 for more details). It is apparent from (15) that the metric induced on $\Im^{+}$from $\widehat{g}\left\lceil\Im^{+}\right.$is degenerate.

(3) A further important remark, arising as a consequence of the requirement 1 in Definition 2.1, is

$$
\Im^{+}=\partial J^{-}(M ; \widehat{M})=\partial M \quad \text { and } \quad \Im^{-}=\partial J^{+}(M ; \widehat{M})=\partial M .
$$

Here is the proof. First, notice that $M=I^{-}(M ; \widehat{M})$. For if there were some $p \in I^{-}(M ; \widehat{M}) \backslash M$, there would also be a continuous timelike past-directed curve from some $q \in M$ to $p$ and it would cut $\partial M$ at some $r$, producing the absurdum $r \in \partial M \cap J^{-}(M ; \widehat{M})=\Im^{+} \cap J^{-}(M ; \widehat{M})=\emptyset$ due to property 1 in def. 2.1. Thus $\Im^{+}:=\partial M=\partial I^{-}(M ; \widehat{M})=\partial J^{-}(M ; \widehat{M})$. The last identity is a general property of causal sets $\partial I^{-}(S)=\partial J^{-}(S)($ see Wa84]).

whose interior and boundary are respectively $M$ and $\Im^{+}$and which is equipped with a Lorentzian smooth metric $\widetilde{g}$ with $\widetilde{g} \uparrow_{M}=\Omega^{2} g$.

${ }^{2}$ Comparing this with DMP06 Mo06, Mo07 the reader should pay attention on the fact that the coordinate $\ell$ was indicated by $u$ in those papers. 
2.3. $F R W$ metrics with $\kappa=0$ and associated asymptotically flat structure. Sticking with (4) where $\kappa=0$, we go to prove that $\left(M, g_{F R W}\right)$ can be extended to a larger spacetime $(\widehat{M}, \widehat{g})$ whenever the function $a$ is suitable. Rescaling the metric $g_{F R W}$ with the conformal factor $\Omega:=a$ which extends smoothly over $\widehat{M}$, we shall also find that, the spacetime $(M, g)$, with $g:=\Omega^{-2} g_{F R W}$, is asymptotically flat at null infinity, admitting $(\widehat{M}, \widehat{g})$ as a conformal completion. This is an important result because, whenever the definition of spacetime asymptotically flat at null infinity applies, it also holds (6)

$$
\Im^{+}=\partial J^{-}(M ; \widehat{M})=\partial M \quad \text { and } \quad \Im^{-}=\partial J^{+}(M ; \widehat{M})=\partial M .
$$

As a consequence of the discussion in remark [2.1. $\Im^{+}$and $\Im^{-}$can be interpreted as a cosmic event horizon and cosmic particle horizon respectively, in common for all observers co-moving with the metric $g_{F R W}$ in $M$. We have, in fact, the following result.

Theorem 2.1. $\quad$ Consider the globally hyperbolic FRW spacetime $\left(M, g_{F R W}\right)$ for $\kappa=0$, with

$$
M \simeq(\alpha, \beta) \times \mathbb{R}^{3}, \quad g_{F R W}=a^{2}(\tau)\left[-d \tau \otimes d \tau+d r \otimes d r+r^{2} d \mathbb{S}^{2}(\theta, \varphi)\right],
$$

$\tau \in(\alpha, \beta)$ and $r, \theta, \phi$ standard spherical coordinates on $\mathbb{R}^{3}$, and there is a constant $\gamma \in \mathbb{R}$ with

$$
a(\tau)=\frac{\gamma}{\tau}+O\left(\frac{1}{\tau^{2}}\right), \quad \frac{d a(\tau)}{d \tau}=-\frac{\gamma}{\tau^{2}}+O\left(\frac{1}{\tau^{3}}\right)
$$

for either $(\alpha, \beta):=(-\infty, 0)$ and $\gamma<0$, or $(\alpha, \beta):=(0,+\infty)$ and $\gamma>0$. The above asymptotic values are meant to be taken as $\tau \rightarrow-\infty$ or $\tau \rightarrow+\infty$ respectively. The following holds.

(a) The spacetime $\left(M, g_{F R W}\right)$ smoothly extends to a larger spacetime $(\widehat{M}, \widehat{g})$, which is a past conformal completion of the asymptotically flat spacetime at past, or future, null infinity, respectively, $\left(M, a^{-2} g_{F R W}\right)$ with $\Omega=a$.

(b) Referring to Def. [2.1, the manifold $M \cup \Im^{ \pm}$has the following further features.

(i) the vector field $\partial_{\tau}$ is a conformal Killing vector for $\widehat{g}$ in $M$ with conformal Killing equation

$$
\mathscr{L}_{\partial_{\tau}} \widehat{g}=-2 \partial_{\tau}(\ln a) \widehat{g} .
$$

where the right-hand side which vanishes approaching $\Im^{ \pm}$.

(ii) $\partial_{\tau}$ tends to become tangent to $\Im^{ \pm}$approaching it and coincides to $-\gamma \widehat{\nabla}^{b} a$ thereon.

(iii) The metric on $\Im^{ \pm}$takes the Bondi form up to the constant factor $\gamma^{2} \neq 0$ :

$$
\widehat{g} \uparrow_{\Im^{+}}=\gamma^{2}\left(-d \ell \otimes d a-d a \otimes d \ell+d \mathbb{S}^{2}(\theta, \varphi)\right),
$$

$\ell \in \mathbb{R}$ being the parameter of the integral lines of $n$.

Proof. The first issue we face is the extension of the spacetime $\left(M, g_{F R W}\right)$ to a larger spacetime $(\widehat{M}, \widehat{g})$. To this end, referring to the metric (4) and the definition (3), let us introduce the new coordinates $u=\tau+r$ and $v=\tau-r$ ranging in subsets of $\mathbb{R}$ individuated by $\tau \in(\alpha, \beta)$ and $r \in(0,+\infty)$. In this way we have

$$
g_{F R W}=a^{2}(\tau(u, v))\left[-\frac{1}{2} d u \otimes d v-\frac{1}{2} d v \otimes d u+\frac{(u-v)^{2}}{4} d \mathbb{S}^{2}(\theta, \varphi)\right] .
$$

Now let us define $U=\tan ^{-1} u$ and $V=\tan ^{-1} v$. Then, per direct substitution, up to a rearrangement of the coefficients into the more convenient form, the above metric can be written as:

$$
g_{F R W}=\frac{a^{2}(\tau(U, V))}{\cos ^{2} U \cos ^{2} V}\left[-\frac{1}{2} d U \otimes d V-\frac{1}{2} d V \otimes d U+\frac{\sin ^{2}(U-V)}{4} d \mathbb{S}^{2}(\theta, \varphi)\right] .
$$




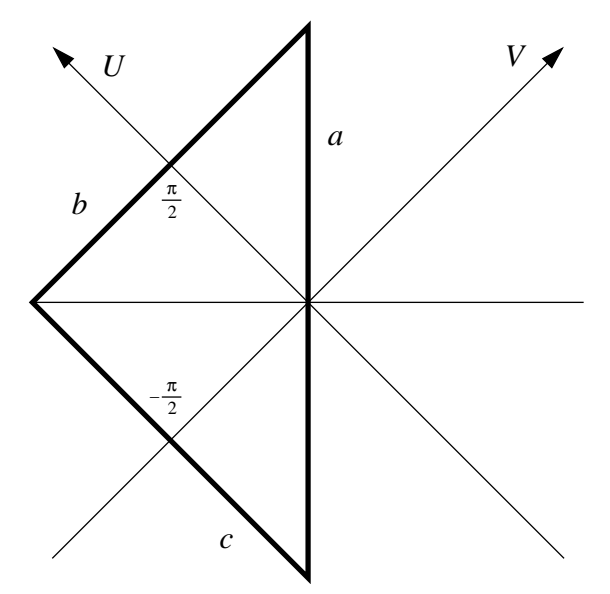

Figure 1: The interior of the triangle represents the original FRW background seen as an open subset of Einstein's static universe. Each point in the $(U, V)$-plane represents a 2-sphere and, furthermore, the segments $b$ and $c$ are respectively $\Im^{+}$and $\Im^{-}$.

Barring the multiplicative coefficient, the coordinate transformations we have performed is the same as one exploits in the standard compactification procedure to switch from Minkowski background to Einstein static universe. The metric, obtained by cancelling the overall factor $a^{2}(\tau(U, V)) /\left(\cos ^{2} U \cos ^{2} V\right)$, is wellbehaved and smooth for $U, V \in \mathbb{R}$ removing the axis $U=V$. However the apparent singularity for $U=V$ is due to the coordinates only; it is nothing but the apparent singularity for $r=0$ in the original metric (4). Consider $\mathbb{R}^{2}$ equipped with null coordinates $U, V$ with respect to the standard Minkowskian metric on $\mathbb{R}^{2}$ and assume that every point is a 2 -sphere with radius $|\sin (U, V)| / 2$ (hence the spheres for $U=V$ are degenerate). Then, let us focus on the segments in $\mathbb{R}^{2}$

$a, V=U$ with $U \in(-\pi / 2, \pi / 2)$,

$b, U=\pi / 2$ with $V \in(-\pi / 2, \pi / 2)$,

$c, V=-\pi / 2$ with $U \in(-\pi / 2, \pi / 2)$.

The original spacetime $M$ is realized as a suitable subset of the union of the segment $a$ (i.e. $r=0$ ) and the interior of the triangle $a b c($ i.e. $r>0)$ as in the figure 1 In this picture it is natural to assume that the null endless segments $b$ and $c$ (which actually represent null 3-hypersurfaces diffeomorphic to $\mathbb{R} \times \mathbb{S}^{2}$ ) individuate $\Im^{+}$and $\Im^{-}$respectively. Notice that, however, it can happen only if $\beta=+\infty$ and, respectively, $\alpha=-\infty$, referring to the domain $(\alpha, \beta)$ of $\tau$. Otherwise the points of $M$ cannot get closer and closer to all the points of those segments. Therefore we are committed to assume $\alpha=-\infty$ and/or $\beta=+\infty$ and we stick with this assumption in the following discussion.

Summarising, we wish to extend $g_{F R W}$ smoothly to a region larger than the open triangle $a b c$ joined with $a$, and including one of the endless segments $b$ and $c$ at least. (The construction implies automatically that, for $M \cup \Im^{+}$and $M \cup \Im^{-}$, the requirement (1) in the definition of asymptotically flat spacetime holds true barring, at most, the causal statement.)

To examine this issue into details we have to specify the form of the function $a$ and study the smoothness of $a^{2}(\tau(U, V)) /\left(\cos ^{2} U \cos ^{2} V\right)$ on the segments $b$ and $c$. For the moment, we stick with a power law

$$
a(\tau)=\gamma \tau^{-k}, \quad \tau \in(\alpha, \beta),
$$

for some integer $k$ and $\gamma \in \mathbb{R} \backslash\{0\}$. These are going to be determined by imposing the smoothness requirement of the metric (9) on $M$ and $\Im^{ \pm}$as well as further requirements arising from the definition 
2.1 in the way we proceed to illustrate. The assumption (10) entails

$$
\frac{a^{2}(\tau(U, V))}{\cos ^{2} U \cos ^{2} V}=2^{2 k} \gamma^{2} \frac{\sin ^{-2 k}(U+V)}{\cos ^{-2 k+2} U \cos ^{-2 k+2} V}
$$

This function is smooth in neighbourhoods of the open segments $b$ and $c$ only if $k \geq 1$. In particular only for $k=1$ this function does not vanish on $b$ and $c$, making nondegenerate $\widehat{g}$ thereon. In these cases, however, a bad singularity appears as soon as $U=-V$ (that is $\tau=0$ ). Therefore we are led to assume that either:

$(\alpha, \beta)=(0,+\infty)$ - and in this case $M(r \geq 0, \tau \in(0,+\infty))$ coincides to the upper half of the triangle $a b c$, and it may be extended to a larger spacetime $(\widehat{M}, \widehat{g})$ by adding a neighbourhood of the endless segment $b$ viewed as $\Im^{+}-$or

$(\alpha, \beta)=(-\infty, 0)-$ and in this case $M(r \geq 0, \tau \in(-\infty, 0))$ coincides to the lower half of the triangle $a b c$, and it may be extended to a larger spacetime $(\widehat{M}, \widehat{g})$ by adding a neighbourhood the endless segment $c$ viewed as $\Im^{-}$.

In both cases the line $U=V$ does not belong to $M$ and to its extension, and the metric $\widehat{g}$ is that in the right-hand side of (9), evaluated in the extended spacetime $\widehat{M}$ :

$$
\widehat{g}=\frac{a^{2}(\tau(U, V))}{\cos ^{2} U \cos ^{2} V}\left[-\frac{1}{2} d U \otimes d V-\frac{1}{2} d V \otimes d U+\frac{\sin ^{2}(U-V)}{4} d \mathbb{S}^{2}(\theta, \varphi)\right] .
$$

The diffeomorphism $\lambda$ is just the inclusion map. We can pass on to check, in both cases, the requirements of the definition 2.1] We have to set up the metric $g$ so that the factor $\Omega$ coincides to $a(\tau(U, V))$. The obtained metric $g$ is nothing but the Minkowski one; therefore it is strongly causal and fulfils Einstein vacuum equations (everywhere) as requested in the condition 2 of definition 2.1 .

We have seen that condition 1 is trivially fulfilled concerning the first two requirements. The last one, $\Im^{+} \cap J^{-}(M ; \widehat{M})=\emptyset$, is true because it is valid when replacing $(\widehat{M}, \widehat{g})$ with Einstein static universe $\left(M_{E}, g_{E}\right)$ (since it is a conformal completion of Minkowski spacetime [Wa84) and $\widehat{g}=c g_{E}$ where $c$ is the nonvanishing factor $a(U, V)^{2} /\left(\cos ^{2} U \cos ^{2} V\right)$, so that $J^{ \pm}\left(M ; M_{E}\right)=J^{ \pm}(M ; \widehat{M})$. Let us pass on to condition 3. First of all we notice that, for $k \geq 1, \Omega$ is smooth in $\widehat{M}$ and vanishes exactly on $\Im^{ \pm}$. Moreover, by direct inspection, we see that

$$
d \Omega=-k \gamma 2^{k}\left(\frac{\sin ^{-k-1}(U+V) d U}{\cos ^{-k+1} U \cos ^{-k-1} V}+\frac{\sin ^{-k-1}(U+V) d V}{\cos ^{-k+1} V \cos ^{-k-1} U}\right) .
$$

To prevent $d \Omega$ from vanishing on $\Im^{ \pm}$, it must hold $k=1$ once again. In this way one finds that

$$
\begin{aligned}
& d \Omega \uparrow_{\Im^{+}}=-2 \gamma d U, \\
& d \Omega \Im_{\Im^{-}}=-2 \gamma d V .
\end{aligned}
$$

We conclude that, assuming (10), all the requirements select $k=1$. Let us pass on to the requirement 4 . By direct inspection one finds that, restricting to $\Im^{+}$and $\Im^{-}$, the Bondi form for the metric $\widehat{g}$ arises

$$
\widehat{g}\left\lceil\Im^{ \pm}=\gamma^{2}\left(-d \ell \otimes d \Omega-d \Omega \otimes d \ell+d \mathbb{S}^{2}(\theta, \varphi)\right) .\right.
$$

where, for arbitrarily fixed constants $k_{+}, k_{-}$:

$$
\begin{array}{lll}
\ell(U)=-\gamma^{-1} \tan U+k_{-}=-\gamma^{-1} u+k_{-} & \text {on } \Im^{-}, \\
\ell(V)=-\gamma^{-1} \tan V+k_{+}=-\gamma^{-1} v+k_{+} & \text {on } \Im^{+} .
\end{array}
$$


In particular, by direct inspection, one may check the validity of the requirement in the definition 2.1 for $\omega_{B}=\gamma^{-2}$ constantly. This also proves the statement (iii) in (b).

The statement (ii) in (b) arises immediately from

$$
\partial_{\tau}=\frac{\partial}{\partial u}+\frac{\partial}{\partial v}=\cos ^{2} U \frac{\partial}{\partial U}+\cos ^{2} V \frac{\partial}{\partial V}=\frac{\partial}{\partial \tan U}+\frac{\partial}{\partial \tan V},
$$

and employing (15) and (16) in the respective cases. The statement (i) in (b) follows immediately from the form of the metric $g_{F R W}$.

From now on, we consider the case $\gamma<0$ only, the other case being analogous. The conditions on $a$ are equivalent to say that, for some $\gamma<0$ and for some $f \in C^{\infty}(-\infty, 0)$ :

$$
a(\tau)=\frac{\gamma}{\tau}+\frac{f(\tau)}{\tau^{2}}>0, \quad \tau \in(-\infty, 0) .
$$

where $f^{\prime}(\tau)=O(1 / \tau)$ as $\tau \rightarrow-\infty$ and $f$ is bounded in a neighbourhood of $-\infty$. With trivial readaptations, all the procedure used above works again and gives rise to (13), (14), (15) and (16) and (8) for $\omega_{B}=1$, by taking a more general form for $a$ than (10). That is, everything holds true if assuming that (17) is valid, where $\gamma<0$ for $(\alpha, \beta):=(-\infty, 0)$ or $\gamma>0$ if $(\alpha, \beta):=(0,+\infty)$ is any constant, and the function $f \in C^{\infty}(\alpha, \beta)$ is bounded in a neighbourhood of $\pm \infty$ with $f^{\prime}(\tau)=O(1 / \tau)$ as $\tau \rightarrow \pm \infty$. This is because the contribution of $f$ cancels on every occurrence as far as the geometry of $\Im^{ \pm}$is concerned.

Remark 2.3. The statements (a),(b) hold true also if we change $g_{F R W}$ smoothly inside a region $M_{0} \simeq(\alpha, \beta) \times \Sigma_{0}$ for a compact $\Sigma_{0} \subset \mathbb{R}^{3}$. (In this case $\partial_{\tau}$ is a conformal Killing vector of the metric at least in $M \backslash M_{0}$.) Indeed, in the said hypotheses, there is an open neighbourhood of $\Im^{ \pm}$where one uses the same construction as above.

2.4. Some examples. As an example, starting with the metric (1) where

$$
a(t)=a_{0} e^{\alpha t} \quad \text { with } a_{0}, \alpha>0 \text { constant, }
$$

one recognises the metric of a conformally static subregion of de Sitter spacetime where the cosmological constant is $\Lambda=3 \alpha^{2}$. This can be considered a realistic model of the observed universe assuming, as done nowadays, that the dark energy dominates among the various sources to gravitation in the framework of Einsteinian theory of gravity. In this case one can fix the integration constant in (3) so that $\tau=-e^{-\alpha t} /\left(a_{0} \alpha\right)$ and thus $\tau \in(-\infty, 0)$. In this case $a(\tau)=-c / \tau$, where $c=1 / \alpha$, and thus we can use the obtained result requiring that $\left(M, g_{F R W}\right)$ admits a cosmological particle horizon in common with all the observers whose world lines are the integral curves of $\partial_{t}$, and that horizon coincides with $\Im^{-}$. A more complicated model is that where, for $c, d>0$ constants,

$$
a(\tau)=-\frac{c}{\tau}+\frac{d}{\tau^{2}}, \quad \tau \in(-\infty, 0) .
$$

Here it is not possible to give the explicit expression for $a=a(t)$, but a straightforward analysis proves that $t \in(-\infty,+\infty)$ and, in this case, one has a strong inflactive behaviour in the remote past $a(t) \sim c e^{t / c}$ as $t \rightarrow-\infty$, whereas $a(t) \sim t^{2} / d$ as $t \rightarrow+\infty$.

Another more complicated example is a spacetime $(M, g)$ with a metric of the form

$$
g(\tau, p)=-\gamma(p)^{2} d t \otimes d t+a(t)^{2} h(p), \quad \text { for }(t, p) \in \mathbb{R} \times \Sigma \simeq M .
$$


(the identification $\mathbb{R} \times \Sigma \simeq M$ being due to $\partial_{t}$ ) where $a(t)$ is as in the previous examples, $\Sigma$ is diffeomorphic to $\mathbb{R}^{3}$ and both $h=d r \otimes d r+r^{2} d \mathbb{S}^{2}$ and $\gamma=1$, outside a compact set in $S$.

As in the previous examples, this spacetime $(M, g)$ is globally hyperbolic provided the Euclidean metric $h$ on $\Sigma$ is complete. This is because $g$ is conformally equivalent to the static metric

$$
g_{0}(\tau, p)=-\gamma(p)^{2} d \tau \otimes d \tau+h(p), \quad \text { for }(\tau, p) \in \mathbb{R} \times \Sigma \equiv M,
$$

and it is known that any spacetime $(M, g)$ with a static metric (20) is globally hyperbolic if there are $c_{1}, c_{2} \in \mathbb{R}$ with $c_{1} \geq \gamma(p) \geq c_{2}>0$ for every $p \in \Sigma$ and $(\Sigma, h)$ is complete Ka78.

\section{General expanding universes with cosmological horizon and the horizon group.}

3.1. Expanding universes with cosmological horizon $\Im^{-}$. The previous discussion has remarked the existence of a nice relationship between asymptotically flat spacetimes at null infinity and expanding FRW spacetimes with a suitable rate of expansion $a$. In particular, $a$ can be interpreted as the rescaling factor $\Omega$ used in asymptotically flat spacetime theory. The new ingredient is, however, the conformal Killing field $\partial_{\tau}$ which, in turn, enjoys a certain interplay with $\Omega$. In asymptotically flat spacetimes at null infinity, an important role DMP06, Mo06, Mo07, in developing quantum field theory, is played by a certain symmetry group of diffeomorphisms defined on $\Im^{+}$, the so called BMS group (e.g., see Wa84]). This group of diffeomorphisms of $\Im^{+}$embodies the isometries of the bulk spacetime [Ge77, AX78] (also the asymptotic ones Wa84), through a suitable geometric correspondence of generators. This fact has a great relevance for applications to quantum field theories [DMP06, Mo06, Mo07. In the following we shall deal with a generalisation of the thesis of theorem 2.1. extracting the relevant geometric structure only, in order to achieve the counterpart of the BMS group for the found class of spacetimes. All the previous examples fulfil the definition below. In particular, the requirements $\Omega \uparrow_{\varsigma^{-}}=0, \Omega^{-1} X(\Omega) \rightarrow 0$ approaching $\Im^{-}$from $M$, can be viewed as a weaker form of the requirements (7), in Theorem 2.1 if assuming $\Omega:=a$ and $X:=\partial_{\tau}$.

Definition 3.1. A globally hyperbolic spacetime $(M, g)$ equipped with a positive smooth function $\Omega$ : $M \rightarrow \mathbb{R}^{+}$and a future-oriented timelike vector $X$ defined on $M$, will be called an expanding universe with geodesically complete cosmological particle horizon when the following facts hold:

1. Existence and causal properties of horizon. $(M, g)$ can be viewed (by means of an isometric embedding) as the interior of a sub manifold-with-boundary of a larger spacetime $(\widehat{M}, \widehat{g})$, the boundary $\Im^{-}:=\partial M$ verifying $\Im^{-} \cap J^{+}(M ; \widehat{M})=\emptyset$.

2. $\Omega-\Im^{+}$interplay. $\Omega$ extends to a smooth function on $\widehat{M}$ such that (i) $\Omega \uparrow_{\Im^{+}}=0$ and (ii) $d \Omega \neq 0$ everywhere on $\Im^{+}$.

3. $X-\Omega-\widehat{g}-\Im^{-}$interplay. $X$ is a conformal Killing vector for $\widehat{g}$ in a neighbourhood of $\Im^{-}$in $M^{3}$, with

$$
\mathscr{L}_{X}(\widehat{g})=-2 X(\ln \Omega) \widehat{g},
$$

where (i) $X(\ln \Omega) \rightarrow 0$ approaching $\Im^{-}$and (ii) $X$ does not tend everywhere to the zero vector approaching $\Im^{-}$.

\footnotetext{
${ }^{3}$ i.e., (21) is valid in $M \cap U$ where $\Im^{-} \subset U \subset \widehat{M}$ for some open set $U$.
} 
4. Bondi-form of the metric on $\Im^{-}$and geodesic completeness. (i) $\Im^{-}$is diffeomorphic to $\mathbb{R} \times \mathbb{S}^{2}$, (ii) the metric $\widehat{g}$ restricted on there takes the Bondi form up to a possible constant factor $\gamma^{2}>0$ :

$$
\left.\widehat{g}\right|_{\Im^{-}}=\gamma^{2}\left(-d \ell \otimes d \Omega-d \Omega \otimes d \ell+d \mathbb{S}^{2}(\theta, \phi)\right),
$$

$d \mathbb{S}^{2}$ being the standard metric on the unit 2-sphere, so that $\Im^{-}$is a null 3-submanifold, and (iii) the curves $\mathbb{R} \ni \ell \mapsto(\ell, \theta, \phi)$ are complete null $\widehat{g}$-geodesics.

The manifold $\Im^{-}$is called the cosmological (particle) horizon of $M$. The integral parameter of $X$ is called the conformal cosmological time. There is a completely analogous definition of contracting universe referring to the existence of $\Im^{+}$in the future instead of $\Im^{-}$.

Remark 3.1. (1) In view of condition 3 , the vector $X$ is a Killing vector of the metric $g_{0}:=\Omega^{-2} g$ in a neighbourhood of $\Im^{-}$in $M$. In this neighbourhood (which may coincide with the whole $M$ ), one can be think of $\Omega^{2}$ as an expansion scale evolving with rate $X\left(\Omega^{2}\right)$ referred to the conformal cosmological time. (2) As shown previously, $\Im^{-} \cap J^{+}(M ; \widehat{M})=\emptyset$ entails $M=I^{+}(M ; \widehat{M})$ and $\Im^{-}=\partial M=\partial I^{+}(M ; \widehat{M})=$ $\partial J^{+}(M ; \widehat{M})$, so that $\Im^{-}$has the proper interpretation as a particle cosmological horizon in common for all the observers in $(M, g)$ evolving along the integral lines of $X$.

(3) It is worth stressing that the spacetimes considered in the given definition are neither homogeneous nor isotropous in general; this is a relevant extension with respect to the family of FRW spacetimes.

(4) Assuming Definition 3.1 the null geodesics in the item 4 are the (complete) integral curves, $\ell$ is an affine parameter and $\widehat{\nabla}^{a} \Omega=-\gamma^{-2}\left(\partial_{\ell}\right)^{a}$ on $\Im^{-}$. The fact that the curves $\mathbb{R} \ni \ell \mapsto(\ell, \theta, \phi)$ are $\widehat{g}$-geodesics is equivalent to say that $\partial_{\Omega} \widehat{g}_{\ell \ell}=0$ everywhere on $\Im^{-}$in the considered coordinates. Finally notice that $\widehat{\Gamma}_{a b}^{\Omega}=0$, for $a, b \neq \Omega$, on $\Im^{-}$therefore $\Im^{-}$is totally geodesic.

(5) In the following, expanding universe with cosmological horizon will mean expanding universe with geodesically complete cosmological particle horizon.

Similarly to the particular case examined previously, also in the general case pictured by Definition 3.1 , the conformal Killing vector field $X$ becomes tangent to $\Im^{-}$and it coincides with $\partial_{\ell}$ up to a nonnegative factor, which now may depend on angular variables, as we go to establish. The proof of the following proposition is in the Appendix.

Proposition 3.1. If $(M, g, \Omega, X, \gamma)$ is an expanding universe with cosmological horizon, the following holds.

(a) X extends smoothly to a (unique) smooth vector field $\widetilde{X}$ on $\Im^{-}$, which may vanish on a closed subset of $\Im^{-}$with empty interior at most. The obtained extension of $X$ to $M \cup \Im^{-}$fulfils the $\widehat{g}$-Killing equation on $\Im^{-}$.

(b) $\widetilde{X}$ has the form $f \partial_{\ell}$, where, referring to the representation $\Im^{+} \equiv \mathbb{R} \times \mathbb{S}^{2}, f$ depends only on the variables $\mathbb{S}^{2}$ and, furthermore, it is smooth and nonnegative.

Since for the FRW spacetimes the function $f=f(\theta, \phi)$ appearing in $\widetilde{X}=f \partial_{\ell}$ holds 1 costantly, the presence of a nontrivial function $f$ is related to the failure of isotropy for the more general spacetimes considered in Definition 3.1

3.2. The horizon symmetry group $G_{\Im^{-}}$. In the forthcoming discussion we shall make use several times of the following technical fact. In the representation $\Im^{-} \equiv \mathbb{R} \times \mathbb{S}^{2} \ni(\ell, s)$, the null $\widehat{g}$-geodesic segments 
imbedded in $\Im^{-}$are all of the curves

$$
J \ni \ell \mapsto(\alpha \ell+\beta, s), \quad \text { for constants } \alpha \neq 0, \beta \in \mathbb{R}, s \in \mathbb{S}^{2} \text {, and some interval } J \subset \mathbb{R} .
$$

We leave the simple proof to the reader.

In this section, assuming that $(M, g)$ satisfies definition 3.1. we select a subgroup $S G_{\Im^{-}}$of isometries of $\Im^{-}$with physical relevance in the following. We shall see in Proposition 3.3 that, as matter of fact, $S G_{\Im^{-}}$contains the isometries generated by Killing vectors obtained as limit towards $\Im^{-}$of (all possible) Killing vectors of $(M, g)$, when these vectors tend to become tangent to $\Im^{-}$.

We have a preliminary proposition about this sort of Killing vectors.

Proposition 3.2. If $(M, g, \Omega, X, \gamma)$ is an expanding universe with cosmological horizon and $Y$ is a Killing vector field of $(M, g), Y$ can be extended to a smooth vector field $\widehat{Y}$ defined on $\widehat{M}$ and the following holds.

(a) $\mathscr{L}_{\widehat{Y}} g=0$ on $M \cup \Im^{-}$;

(b) $\widetilde{Y}:=\widehat{Y} \uparrow_{\Im^{-}}$is uniquely determined by $Y$,

(c) $\widetilde{Y}$ is tangent to $\Im^{-}$if and only if $g(Y, X)$ vanishes approaching $\Im^{-}$from $M$.

Restricting to the linear space of the Killing fields $Y$ on $(M, g)$ such that $g(Y, X) \rightarrow 0$ approaching $\Im^{-}$, the following further facts hold.

(d) If $\widetilde{Y}$ vanishes in some $A \subset \Im^{-}$and $A \neq \emptyset$ is open (with respect to the topology of $\Im^{-}$), then $Y=0$ everywhere in $M$ and, thus, $\widehat{Y}=0$ everywhere in $M \cup \Im^{+}$.

(e) The linear map $Y \mapsto \widetilde{Y}$ is injective.

The proof of the proposition above is given in the Appendix.

The statements (a) and (c) of Proposition 3.2 establish that the Killing vectors $Y$ in $M$ with $(Y, X) \rightarrow 0$ approaching $\Im^{-}$extend to Killing vectors of $\left(\Im^{-}, h\right), h$ being the degenerate metric on $\Im^{-}$induced by $\widehat{g}$. It is worth remarking that these Killing vectors of $(M, g)$ are represented on $\Im^{-}$faithfully due to (e): The boundary $\Im^{-}$embodies the full information about these symmetries of the bulk $M$.

Since the vector fields $\widehat{Y}$ tangent to $\Im^{-}$admit $\Im^{-}$as invariant manifold, the following definition is appropriate in view of (c) in Proposition 3.2 .

Definition 3.2. If $(M, g, \Omega, X, \gamma)$ is an expanding universe with cosmological horizon, a Killing vector field of $(M, g), Y$, is said to to preserve $\Im^{-}$if $g(Y, X) \rightarrow 0$ approaching $\Im^{-}$. Similarly, the Killing isometries of the (local) one-parameter group generated by $Y$ are said to preserve $\Im^{-}$.

In the rest of this part we shall consider the one-parameter group of isometries of $\left(\Im^{-}, h\right)$ generated by such Killing vectors $\widehat{Y} \uparrow_{\Im^{-}}$. These isometries amount to a very little part of the huge group of isometries of $\left(\Im^{-}, h\right)$. For instance, referring to the representation $(\ell, s) \in \mathbb{R} \times \mathbb{S}^{2} \equiv \Im^{-}$, for every smooth diffeomorphism $f: \mathbb{R} \rightarrow \mathbb{R}$, the transformation

$$
\ell \rightarrow f(\ell), \quad s \rightarrow s
$$

is an isometry of $\left(\Im^{-}, h\right)$. However only diffeomorphisms of the form $f(\ell)=a \ell+b$ with $a \neq 0$ can be isometries generated by the restriction $\widehat{Y} \varsigma_{\varsigma^{-}}$to $\Im^{-}$of extensions of Killing fields $Y$ of $(M, g)$ as in the proposition 3.2. This is because those isometries are restrictions of isometries of the manifolds-withboundary $\left(M \cup\left\{\Im^{-}\right\}, \widehat{g}_{\left.\left.\right|_{M \cup\left\{\Im^{-}\right.}\right)}\right)$, and thus they preserve the null $\widehat{g}$-geodesics in $\Im^{-}$. These geodesics have the form (23). The requirement that, for every constants $a, b \in \mathbb{R}, a \neq 0$, there must be constants $a^{\prime}, b^{\prime} \in \mathbb{R}, a^{\prime} \neq 0$ such that $f(a \ell+b)=a^{\prime} \ell+b^{\prime}$ for all $\ell$ varying in a fixed (nonempty) interval $J$, is 
fulfilled only if $f$ is an affine transformation as said above.

Relaxing the constraints on angular variables in (24), i.e. allowing as well transformations of angular coordinates too, let us such a restricted class of isometries of $\Im^{-}$. Afterwards we shall come back to the Killing vectors tangent to $\Im^{-}$introduced in Proposition 3.2. Therefore we aim to study the class $G_{\Im^{+}}$of diffeomorphisms $F: \Im^{-} \rightarrow \Im^{-}$

$$
\ell \rightarrow \ell^{\prime}:=f(\ell, s), \quad s \rightarrow s^{\prime}:=g(\ell, s) \quad \text { with } \ell \in \mathbb{R} \text { and } s \in \mathbb{S}^{2},
$$

such that: (i) they are isometries of the degenerate metric $h$ induced by $\widehat{g} \uparrow_{\Im^{-}}(\mathbf{2 2})$ and (ii) they may be restrictions to $\Im^{-}$of isometries of $\widehat{g}$ in $M \cup \Im^{-}$.

Assume that $F \in G_{\Im^{-}}$. The curve $\gamma: \mathbb{R} \ni \ell \rightarrow \gamma_{s}(\ell) \equiv(\ell, s)$ (with $s \in \mathbb{S}^{2}$ arbitrarily fixed) is a null geodesic forming $\Im^{-}$, therefore $\mathbb{R} \ni \ell \rightarrow F\left(\gamma_{s}(\ell)\right)$ has to be, first of all, a null curve. In other words

$$
\widehat{g} \uparrow_{\Im^{-}}\left(\frac{\partial f}{\partial \ell} \frac{\partial}{\partial \ell}+\frac{\partial g}{\partial \ell} \frac{\partial}{\partial \theta}+\frac{\partial g}{\partial \ell} \frac{\partial}{\partial \phi}, \frac{\partial f}{\partial \ell} \frac{\partial}{\partial \ell}+\frac{\partial g}{\partial \ell} \frac{\partial}{\partial \theta}+\frac{\partial g}{\partial \ell} \frac{\partial}{\partial \phi}\right)=0 .
$$

Using (22) and arbitrariness of $s \equiv(\theta, \phi)$, it implies that $g$ does not depend on $\ell$ since the standard metric on the unital sphere is strictly positive defined. The map $g$ has to be an isometry of $\mathbb{S}^{2}$ equipped with its standard metric. In other words $g \in O(3)$. Moreover, $\mathbb{R} \ni \ell \rightarrow F\left(\gamma_{s}(\ell)\right)=(f(\ell, s), g(s))$ has to be a null geodesic which belongs to $\Im^{-}$. As a consequence of (2) in remark 3.1 $(f(\ell, s), g(s))=(c(s) \ell+b(s), g(s))$ for some fixed numbers $c(s), b(s) \in \mathbb{R}$ with $c(s)>0$, and for every $\ell \in \mathbb{R}$. Summarising, if a smooth $\Im^{-}-$ diffeomorphism $F$ as in (25) fulfils (i) and (ii), it must be $g(\ell, s)=R(s)$ for all $\ell, s$ and $f(\ell, s)=c(s) \ell+b(s)$, for all $\ell, s$, for some $R \in O(3), c, b \in C^{\infty}\left(\mathbb{S}^{2}\right)$ with $c(s) \neq 0$. It is obvious that, conversely, every such a diffeomorphism fulfils (i) and (ii), so that we have characterised the class of isometries verifying (i) and (ii) completely.

Remark 3.2. (1) By direct inspection one sees that the class $G_{\Im^{-}}$of all diffeomorphisms $F$ as above is a group with respect to the composition of diffeomorphisms.

(2) A transformations $F \in G_{\Im^{-}}$, associated with $R \in O(3)$, but $R \notin S O(3)$, cannot belong to any oneparameter group of isometries induced by Killing vectors in $M$. This is because $R$ should be continuously connected to the identity rotation $I$ (that reached by the vanishing value of the one-parameter group). This would be impossible if $R \notin S O(3)$.

(3) In the following, each element of the one-parameter group of diffeomorphisms generated by a vector field $Z$ will be denoted by $\exp \{t Z\}$, where $t \in \mathbb{R}$ is the parameter.

From now on we shall restrict ourselves to the subgroup of $G_{\Im^{-}}$whose elements are constructed using elements of $S O(3)$. We give the following definitions.

Definition 3.3. $\quad$ The horizon symmetry group $S G_{\Im^{-}}$is the group (with respect to the composition of functions) of all diffeomorphisms of $\mathbb{R} \times \mathbb{S}^{2}$,

$$
F_{(a, b, R)}: \mathbb{R} \times \mathbb{S}^{2} \ni(\ell, s) \mapsto\left(e^{a(s)} \ell+b(s), R(s)\right) \in \mathbb{R} \times \mathbb{S}^{2} \quad \text { with } \ell \in \mathbb{R} \text { and } s \in \mathbb{S}^{2},
$$

where $a, b \in C^{\infty}\left(\mathbb{S}^{2}\right)$ are arbitrary smooth functions and $R \in S O(3)$.

The Horizon Lie algebra $\mathfrak{g}_{\Im^{-}}$is the infinite-dimensional Lie algebra of smooth vector fields on $\mathbb{R} \times \mathbb{S}^{2}$ generated by the fields

$$
S_{1}, S_{2}, S_{3}, \beta \partial_{\ell}, \ell \alpha \partial_{\ell}, \quad \text { for all } \alpha, \beta \in C^{\infty}\left(\mathbb{S}^{2}\right) \text {. }
$$


$S_{1}, S_{2}, S_{3}$ indicate the three smooth vector fields on the unit sphere $\mathbb{S}^{2}$ generating rotations about the orthogonal axes, respectively, $x, y$ and $z$. (Since, looking at (20), $\Im^{-}$is naturally isometric to $\mathbb{R} \times \mathbb{S}^{1}$, these fields can be viewed as fields on $\Im^{-}$).

It is worth noticing that $S G_{\Im^{-}}$depends on the geometric structure of $\Im^{-}$but not on the attached spacetime $(M, g)$, which, in principle, could not admit any Killing vector preserving $\Im^{-}$. In this sense it is a universal object for the whole class of expanding spacetimes with cosmological horizon. $S G_{\Im^{-}}$may be seen as an abstract group defined on the set $S O(3) \times C^{\infty}\left(\mathbb{S}^{2}\right) \times C^{\infty}\left(\mathbb{S}^{2}\right)$, without reference to any expanding spacetime with cosmological horizon $(M, g)$. Adopting this point of view, if we indicate $F_{a, b, R}$ by the abstract triple $(R, a, b)$, the composition between elements in $S G_{\Im^{-}}$reads

$$
\begin{aligned}
& (R, a, b)\left(R^{\prime}, a^{\prime}, b^{\prime}\right)=\left(R R^{\prime}, a^{\prime}+a \circ R^{\prime}, e^{a \circ R^{\prime}} b^{\prime}+b \circ R^{\prime}\right) \\
& \text { for all }(R, a, b),\left(R^{\prime}, a^{\prime}, b^{\prime}\right) \in S O(3) \times C^{\infty}\left(\mathbb{S}^{2}\right) \times C^{\infty}\left(\mathbb{S}^{2}\right) .
\end{aligned}
$$

where $\circ$ denotes the usual composition of functions.

The relationship between $S G_{\Im^{-}}$and $\mathfrak{g}_{\Im^{-}}$is clarified in the following proposition. In a sense, (b) states that $\mathfrak{g}_{\Im^{-}}$could be considered as the Lie algebra of $S G_{\Im^{-}}$. This fact could be investigated further if endowing $S G_{\Im^{-}}$with the structure of an infinite-dimensional Lie group; however we shall not address this issue here.

Proposition 3.3. Referring to the definition 3.3, the following facts hold:

(a) Each vector field $Z \in \mathfrak{g}_{\Im^{-}}$is complete and the generated (global) one-parameter group of diffeomorphisms of $\mathbb{R} \times \mathbb{S}^{2},\{\exp \{t Z\}\}_{t \in \mathbb{R}}$, is a subgroup of $S G_{\Im^{-}}$.

(b) For every $F \in S G_{\Im^{-}}$there are $Z_{1}, Z_{2} \in \mathfrak{g}_{\Im^{-}}-$with, possibly, $Z_{1}=Z_{2}$ - such that $F=$ $\exp \left\{t_{1} Z_{1}\right\} \exp \left\{t_{2} Z_{2}\right\}$ for some real numbers $t_{1}, t_{2}$.

The proof of this proposition is in the Appendix.

Furthermore, we have the following important result which finally makes explicit the interplay of Killing vectors $Y$ in $M$ preserving $\Im^{-}$, the group $S G_{\Im^{-}}$and the Lie algebra $\mathfrak{g}_{\Im^{-}}$.

Theorem 3.1. Let $(M, g, \Omega, X, \gamma)$ be an expanding universe with cosmological horizon and $Y$ a Killing vector field of $(M, g)$ preserving $\Im^{-}$. The following holds.

(a) The unique smooth extension $\widetilde{Y}$ of $Y$ to $\Im^{-}$(see Proposition [.2.2) belongs to $\mathfrak{g}_{\Im^{-}}$.

(b) $\{\exp \{t \tilde{Y}\}\}_{t \in \mathbb{R}}$ is a subgroup of $S G_{\Im^{-}}$.

The proof of this theorem is in the Appendix.

As an example consider the expanding universe $M$ with cosmological horizon associated with the metric $g_{F R W}$ (2) with $\kappa=1$ and $a$ as in (a) of Theorem 2.1. In this case $X:=\partial_{\tau}$ and there are a lot of Killing vectors $Y$ of $\left(M, g_{F R W}\right)$ satisfying $g_{F R W}(Y, X) \rightarrow 0$ approaching $\Im^{-}$. The most trivial ones are all of the Killing vectors of the surfaces at $\tau=$ constant with respect to the induced metric. We have here a Lie algebra generated by 6 independent Killing vectors $Y$ generating, respectively, space translations and space rotations. In this case $g_{F R W}(Y, X)=0$ so that the associated Killing vectors $\widehat{Y} \uparrow_{\Im^{-}}$belongs to $\mathfrak{g}_{\Im^{-}}$. This is not the whole story in the sharp case $a(\tau)=\gamma / \tau$ with $\gamma<0$ (and this corresponds to the expanding de Sitter spacetime). Indeed, in that case, there is another Killing vector $B$ of $g_{F} R W$ fulfilling $g_{F R W}(B, X) \rightarrow 0$ approaching $\Im^{-}$. It is $B:=\tau \partial_{\tau}+r \partial_{r} . B$, extended to $M \cup \Im^{-}$, gives rise to the structure of a bifurcate Killing horizon KW91]. 
A last technical result, which will be useful in discussing the positivity of the energy of a quantum state we shall introduce later, is the following. The proof is in the Appendix.

Proposition 3.4. Let $(M, g, \Omega, X, \gamma)$ be an expanding universe with cosmological horizon and $Y$ a smooth vector field of $(M, g)$ which tends to the smooth field $\widetilde{Y} \in \mathfrak{g}_{\Im^{-}}$pointwisely. (In particular it happens when $Y$ is a Killing vector of $(M, g)$ preserving $\Im^{-}$or for $\left.Y=X\right)$.

If there is an open set $A \subset \widehat{M}$ with $A \supset \Im^{-}$and such that $\left.Y\right|_{A \cap M}$ is timelike and future directed, then, everywhere on $\Im^{-}$,

$$
\tilde{Y}(\ell, s)=f(s) \partial_{\ell}, \quad \text { for some } f \in C^{\infty}\left(\mathbb{S}^{2}\right) \text {, with } f(s) \geq 0 \text { on } \mathbb{S}^{2} .
$$

\section{Linear Quantum Fields and preferred states induced by the cosmological horizon.}

In this section $(M, g, \Omega, X, \gamma)$ is an expanding universe with cosmological horizon. Since $(M, g)$ is globally hyperbolic per definition, one can study properties of quantum fields propagating therein, following the algebraic approach in the form presented in KW91, Wa94] (see also the Appendix B for a very short summary of main tools).

4.1. QFT in the bulk. Consider linear bosonic QFT in $(M, g)$ based on the symplectic space $\left(\mathcal{S}(M), \sigma_{M}\right)$, where $\mathcal{S}(M)$ is the space of real smooth, compactly supported on Cauchy surfaces, solutions $\varphi$ of a generally massive Klein-Gordon equation in $M$ :

$$
P \phi=0, \quad \text { where } P \text { is the Klein-Gordon operator } P=\square+\xi R+m^{2} .
$$

with $\square=-\nabla_{a} \nabla^{a}, m>0$ and $\xi \in \mathbb{R}$ constants. The nondegenerate, Cauchy-surface independent, symplectic form $\sigma_{M}$ is:

$$
\sigma_{M}\left(\varphi_{1}, \varphi_{2}\right):=\int_{S}\left(\varphi_{2} \nabla_{N} \varphi_{1}-\varphi_{1} \nabla_{N} \varphi_{2}\right) d \mu_{g}^{(S)}
$$

$S$ being any Cauchy surface of $M$ with normal unit future-directed vector $N$ and 3-volume measure $d \mu_{g}^{(S)}$ induced by $g$.

As is well known BR021, BR022, it possible to associate canonically any symplectic space, for instance $\left(\mathrm{S}(M), \sigma_{M}\right)$, with a Weyl $C^{*}$-algebra, $\mathcal{W}(M)$ in this case. This is the, unique up to (isometric) *isomorphisms, $C^{*}$-algebra with generators $W_{M}(\varphi) \neq 0, \varphi \in \mathrm{S}(M)$, satisfying Weyl commutation relations (we use here conventions adopted in Wa94)

$$
W_{M}(-\varphi)=W_{M}(\varphi)^{*}, \quad W_{M}(\varphi) W_{M}\left(\varphi^{\prime}\right)=e^{i \sigma_{M}\left(\varphi, \varphi^{\prime}\right) / 2} W\left(\varphi+\varphi^{\prime}\right) .
$$

$\mathcal{W}(M)$ represents the basic set of quantum observable associated with the bosonic field $\phi$ propagating in the bulk spacetime $(M, g)$. The generators $W_{M}(\varphi)$ are formally interpreted as the exponentials $e^{-i \sigma_{M}(\Phi, \varphi)}$ where $\sigma_{M}(\Phi, \varphi)=-\sigma_{M}(\varphi, \Phi)$ is the field operator symplectically smeared with a solution $\varphi \in \mathcal{S}(M)$ of field equations (concerning the sign of $\sigma$ we employ conventions used in Wa94 which differ from those adopted in [KW91]). The interpretation has a rigorous meaning referring to a GNS representation Haa92, BR021 of $\mathcal{W}(M)$ induced by an algebraic state $\omega$. Indeed, if the considered state $\omega$ is regular, 
the unitary group $\mathbb{R} \ni t \mapsto \Pi_{\omega}(W(t \psi))$ is strongly continuous and $-i \sigma_{M}(\Phi, \varphi)$ can be defined as its self-adjoint generator.

The more usual field operator $\Phi(f)$ smeared with functions $f \in C_{0}^{\infty}(M)$ is related with $\sigma_{M}(\Phi, \varphi)$ by means of $\Phi(f):=\sigma_{M}(\Phi, E(f))$, where now $E$ denotes the causal propagator $E: C_{0}^{\infty}(M) \rightarrow C^{\infty}(M)$. That is the difference of the advanced and retarded fundamental solutions of Klein-Gordon equation which exist in every globally hyperbolic spacetime [Le53, Di80, BGP96. $\Phi$ solves Klein-Gordon equation in distributional sense: $\Phi(P f)=0$ because $P=P^{t}$ and $P \circ E=0$ by definition.

The main goal of this section is to prove that the geometric structures on $(M, g, \Omega, X, \gamma)$ pick out a very remarkable algebraic state $\omega$ on $\mathcal{W}(M)$, which, among other properties turns out to be invariant under the natural action of every Killing isometry of $(M, g)$ which preserves $\Im^{-}$. This happens provided a certain algebraic interplay of QFT in $M$ and QFT on $\Im^{-}$exists.

4.2. Bosonic $Q F T$ on $\Im^{-}$and $G_{\Im^{-}}$-invariant states. Referring to $\Im^{-} \equiv \mathbb{R} \times \mathbb{S}^{2}$, consider the real symplectic space $\left(\mathrm{S}\left(\Im^{+}\right), \sigma\right)$, where

$$
\mathrm{S}\left(\Im^{+}\right):=\left\{\psi \in C^{\infty}\left(\mathbb{R} \times \mathbb{S}^{2}\right) \mid \psi, \partial_{\ell} \psi \in L^{2}\left(\mathbb{R} \times \mathbb{S}^{2}, d \ell \wedge \epsilon_{\mathbb{S}^{2}}(\theta, \phi)\right\},\right.
$$

$\epsilon_{\mathbb{S}^{2}}$ being the standard volume form of the unit 2-sphere, and the nondegenerate symplectic form $\sigma$ is given by, if $\psi_{1}, \psi_{2} \in \mathbf{S}\left(\Im^{+}\right)$

$$
\sigma\left(\psi_{1}, \psi_{2}\right):=\int_{\mathbb{R} \times \mathbb{S}^{2}}\left(\psi_{2} \frac{\partial \psi_{1}}{\partial \ell}-\psi_{1} \frac{\partial \psi_{2}}{\partial \ell}\right) d \ell \wedge \epsilon_{\mathbb{S}^{2}}(\theta, \phi) .
$$

As before, there is a, unique up to (isometric) *-isomorphisms, $C^{*}$-algebra with generators $W(\psi) \neq 0$, $\psi \in \mathrm{S}\left(\Im^{-}\right)$, satisfying Weyl commutation relations

$$
W(-\psi)=W(\psi)^{*}, \quad W(\psi) W\left(\psi^{\prime}\right)=e^{i \sigma\left(\psi, \psi^{\prime}\right) / 2} W\left(\psi+\psi^{\prime}\right),
$$

that is the Weyl $C^{*}$-algebra $\mathcal{W}\left(\Im^{-}\right)$associated with $\left(\mathrm{S}\left(\Im^{-}\right), \sigma\right)$.

Remark 4.1. Exploiting the given definitions, it is simply proved that $\left(\mathrm{S}\left(\Im^{+}\right), \sigma\right)$ is invariant under the pull-back action of $G_{\Im^{-}}$. In other words the following hold (i) $\psi \circ g \in \mathrm{S}\left(\Im^{+}\right)$if $\psi \in \mathrm{S}\left(\Im^{+}\right)$and also (ii) $\sigma\left(\psi_{1} \circ g, \psi_{2} \circ g\right)=\sigma\left(\psi_{1}, \psi_{2}\right)$ for all $g \in G_{\Im^{-}}$and $\psi_{1}, \psi_{2} \in \mathbf{S}\left(\Im^{+}\right)$. As a well known consequence BR022, BGP96, $G_{\Im^{-}}$induces a $*$-automorphism $G_{\Im^{-}}$-representation $\alpha: \mathcal{W}\left(\Im^{-}\right) \rightarrow \mathcal{W}\left(\Im^{-}\right)$, uniquely individuated (by linearity and continuity) by the requirement

$$
\alpha_{g}(W(\psi)):=W\left(\psi \circ g^{-1}\right), \quad \psi \in \mathrm{S}\left(\Im^{+}\right) \text {and } g \in G_{\Im^{-}} .
$$

Since we are interested in physical properties which are $G_{\Im^{-}}$-invariant, we face the issue about the existence of $G_{\Im^{-}}$-invariant algebraic states on $\mathcal{W}\left(\Im^{-}\right)$. Obviously, $G_{\Im^{-}}$-invariance is here referred to the representation $\alpha$ of $G_{\Im^{-}}$.

4.3. $G_{\Im^{-}}$-invariant algebraic states on $\mathcal{W}\left(\Im^{-}\right)$. We adopt here the definition of quasifree state given in [KW91, and also adopted in DMP06, Mo06, Mo07, summarised in the Appendix B. Consider the quasifree state $\lambda$ defined on $\mathcal{W}\left(\mathrm{S}\left(\Im^{+}\right)\right)$uniquely induced by linearity and continuity from; if $\psi, \psi^{\prime} \in \mathrm{S}\left(\Im^{+}\right)$ then

$$
\lambda(W(\psi))=e^{-\mu(\psi, \psi) / 2}, \quad \mu\left(\psi, \psi^{\prime}\right):=\operatorname{Re} \int_{\mathbb{R}^{2} \mathbb{S}^{2}} 2 k \Theta(k) \overline{\widehat{\psi}(k, \theta, \phi)} \widehat{\psi}^{\prime}(k, \theta, \phi) d k \wedge \epsilon_{\mathbb{S}^{2}}(\theta, \phi),
$$


the bar denoting the complex conjugation, $\Theta(k):=0$ for $k<0$ and $\Theta(k):=1$ for $k \geq 0$; here we have used the $\ell$-Fourier-Plancherel transform $\widehat{\psi}$ of $\psi$ :

$$
\widehat{\psi}(k, \theta, \phi):=\int_{\mathbb{R}} \frac{e^{i k \ell}}{\sqrt{2 \pi}} \psi(\ell, \theta, \phi) d \ell, \quad(k, \theta, \phi) \in \mathbb{R} \times \mathbb{S}^{2} .
$$

The constraint

$$
\left|\sigma\left(\psi, \psi^{\prime}\right)\right|^{2} \leq 4 \mu(\psi, \psi) \mu\left(\psi^{\prime}, \psi^{\prime}\right), \quad \text { for every } \psi, \psi^{\prime} \in \mathrm{S},
$$

which has to hold for every quasifree state (see Appendix B), is fulfilled by the scalar product $\mu$, as the reader can verify by inspection, since it results:

$$
\sigma\left(\psi_{1}, \psi_{2}\right)=-2 \operatorname{Im}\left\{\int_{\mathbb{R} \times \mathbb{S}^{2}} 2 k \Theta(k) \overline{\widehat{\psi}_{1}(k, \theta, \phi)} \widehat{\psi}_{2}(k, \theta, \phi) d k \wedge \epsilon_{\mathbb{S}^{2}}(\theta, \phi)\right\}, \text { for all } \psi_{1}, \psi_{2} \in \mathrm{S}\left(\Im^{-}\right) .
$$

Consider the GNS representation of $\lambda,(\mathfrak{H}, \Pi, \Upsilon)$. Since $\lambda$ is quasifree, $\mathfrak{H}$ is a bosonic Fock space $\mathcal{F}_{+}(\mathcal{H})$ with cyclic vector $\Upsilon$ given by the Fock vacuum and 1-particle Hilbert $\mathcal{H}$ space obtained as the Hilbert completion of the complex space generated by the "positive-frequency parts" $\Theta \widehat{\psi}=: K_{\mu} \psi$, of every wavefunction $\psi \in \mathrm{S}\left(\Im^{-}\right)$, with the scalar product $\langle\cdot, \cdot\rangle$ individuated by $\mu$, as stated in (ii) of Lemma B.1 in the Appendix B. In our case it reads,

$$
\left\langle K_{\mu} \psi, K_{\mu} \psi^{\prime}\right\rangle=\int_{\mathbb{R} \times \mathbb{S}^{2}} 2 k \Theta(k) \overline{\widehat{\psi}(k, \theta, \phi)} \widehat{\psi}^{\prime}(k, \theta, \phi) d k \wedge \epsilon_{\mathbb{S}^{2}}(\theta, \phi) .
$$

Notice that it has to be extended by (anti-)linearity and continuity to $\mathcal{H}$. The so-defined map $K_{\mu}$ : $\mathrm{S}\left(\Im^{-}\right) \rightarrow \mathcal{H}$ is $\mathbb{R}$-linear and has a dense complexified range. As $\lambda$ is quasifree, it is regular, so that symplectically-smeared field operators $\sigma(\Psi, \psi)$ are defined in $\mathcal{F}_{+}(\mathcal{H})$ via Stone's theorem, namely $\Pi(W(t \psi))=e^{-i t \sigma(\Psi, \psi)}$ with $t \in \mathbb{R}$ and $\psi \in \mathrm{S}\left(\Im^{-}\right)$, and these operators have the usual form (95) in terms of creation and annihilation operators.

A state similar to $\lambda$, and denoted by the same symbol, was defined on $\Im^{+} \simeq \mathbb{R} \times \mathbb{S}^{2}$ in DMP06, Mo06, $\mathrm{Mo07}^{4}$, for asymptotically flat spacetimes at future null infinity, where the coordinate $\ell$ was indicated by $u$ and complex coordinates $\zeta, \bar{\zeta}$, instead of $(\theta, \phi)$, were employed on $\mathbb{S}^{2}$. In the appropriate coordinates, that state enjoys the form (36) exactly. Therefore, making use of Theorem 2.12 in [DMP06, we have the following straightforwardly result.

Proposition 4.1. The quasifree state $\lambda$ on $\mathcal{W}\left(\Im^{-}\right)$, defined in (36) is pure. In other words, the GNS representation $\Pi$ of $\lambda$ is irreducible and, equivalently, the range of the $\mathbb{R}$-linear map $K_{\mu}: \mathrm{S}\left(\Im^{-}\right) \ni \psi \rightarrow$ $\psi_{+} \in \mathcal{H}$ is dense. Finally, the one-particle space $\mathcal{H}$ of its GNS representation is isomorphic to the separable Hilbert space $L^{2}\left(\mathbb{R}^{+} \times \mathbb{S}^{2} ; 2 k d k \wedge \epsilon_{\mathbb{S}^{2}}\right)$.

The state $\lambda$ enjoys further remarkable properties in reference to the group $G_{\Im^{-}}$. The first theorem concerns invariance properties of $\lambda$.

Theorem 4.1. The state $\lambda$ defined in (36), and with GNS triple $(\mathfrak{H}, \Pi, \Upsilon)$, satisfies the following. (a) It is invariant under the $*$-automorphisms representation $G_{\Im^{-}} \ni g \mapsto \alpha_{g}$, in other words: $\lambda\left(\alpha_{g}(a)\right)=$ $\lambda(a)$ for all $a \in \mathcal{W}\left(\Im^{-}\right)$and $g \in G_{\Im^{-}}$.

\footnotetext{
${ }^{4}$ In [DMP06, Mo06] a different, but unitarily-equivalent, Hilbert space representation was used referring to the measure $d k$ instead of $2 k d k$. Features of Fourier-Plancherel theory on $\mathbb{R} \times \mathbb{S}^{2}$ were discussed in the Appendix C of [Mo07].
} 
(b) The unique unitary representation $U: G_{\Im^{-}} \ni g \mapsto U_{g}$ that implements $\alpha$ in $\mathfrak{H}$ leaving $\Upsilon$ invariant, that is,

$$
U_{g} a U_{g}^{*}=\alpha_{g}(a) \quad \text { and } \quad U_{g} \Upsilon=\Upsilon \quad \text { for all } a \in \mathcal{W}\left(\Im^{-}\right) \text {and } g \in G_{\Im^{-}},
$$

leaves $\mathcal{H}$ invariant and it is determined by $U \uparrow_{\mathcal{H}}$ completely. $U$ has the tensorialised form

$$
U=I \oplus U \uparrow_{\mathcal{H}} \oplus\left(U \uparrow_{\mathcal{H}} \otimes U \uparrow_{\mathcal{H}}\right) \oplus\left(U \uparrow_{\mathcal{H}} \otimes U \uparrow_{\mathcal{H}} \otimes U \uparrow_{\mathcal{H}}\right) \oplus \cdots
$$

(c) The unitary representation $U \uparrow_{\mathcal{H}}: \mathcal{H} \rightarrow \mathcal{H}$ is irreducible.

Proof. (a) If $\psi \in \mathrm{S}\left(\Im^{-}\right)$is a wavefunction, we can write that

$$
\left(K_{\mu} \psi\right)(k, \theta, \phi):=\int_{\mathbb{R}} \frac{e^{i k \ell}}{\sqrt{2 \pi}} \psi(\ell, \theta, \phi) d k, \quad(k, \theta, \phi) \in \mathbb{R}^{+} \times \mathbb{S}^{2},
$$

and the complex linear span of these vectors is dense in $\mathcal{H}$. It is simple to introduce a unitary representation $V$ of $G_{\Im^{-}}$on $\mathcal{H}$, by defining, if $g=(R, a, b) \in G_{\Im^{-}}$and $s=(\theta, \phi)$

$$
\left(V_{(R, a, b)} \varphi\right)(k, s):=e^{a\left(R^{-1}(s)\right)} e^{-i k b\left(R^{-1}(s)\right)} \varphi\left(e^{a\left(R^{-1}(s)\right)} k, R^{-1}(s)\right) \quad \text { for all } \varphi \in \mathcal{H} .
$$

This representation arises from (42) by requiring that $V_{g}$ satisfies

$$
K_{\mu}\left(\psi \circ g^{-1}\right)=V_{g} K_{\mu} \psi, \quad \text { for every } \psi \in \mathrm{S}\left(\Im^{-}\right) \text {and } g \in G_{\Im^{-}} .
$$

Using (36) and (40), the first consequence of (43) and of the unitarity of $V$ is that

$$
\begin{gathered}
\lambda\left(\alpha_{g}(W(\psi))\right)=\lambda\left(W\left(\psi \circ g^{-1}\right)\right)=e^{-\operatorname{Re}\left\langle V_{g} K_{\mu} \psi, V_{g} K_{\mu} \psi\right\rangle / 2} \\
=e^{-\operatorname{Re}\left\langle K_{\mu} \psi, K_{\mu} \psi\right\rangle / 2}=\lambda(W(\psi))
\end{gathered}
$$

By linearity and continuity it implies immediately that $\lambda$ is invariant under $G_{\Im^{-}}$as soon as it arises $\lambda\left(\alpha_{g}(a)\right)=\lambda(a)$ for every $a \in \mathcal{W}\left(\Im^{-}\right)$.

(b) As it is well known Ar99, since $\lambda$ is invariant under $G_{\Im^{-}}$through $\alpha$, there is a unique unitary representation of $G_{\Im^{-}}$in the GNS representation of $\lambda$ that implements $\alpha$ and leaves $\Upsilon$ fixed. Let us determine such a representation. We remind the reader that, the symplectically smeared field operators are defined in $\mathfrak{H}=\mathcal{F}_{+}(\mathcal{H})$ as (see Appendix B)

$$
\sigma(\psi, \Psi):=i a\left(K_{\mu} \psi\right)-i a^{\dagger}\left(K_{\mu} \psi\right), \quad \text { for all } \psi \in \mathrm{S}
$$

$a(\phi)$ and $a^{\dagger}(\phi), \phi \in \mathcal{H}_{\mu}$, being the usual annihilation (antilinear in $\phi$ ) and creation operators defined in the dense linear manifold spanned by the states with finite number of particles. This identity, together with (44), implies that

$$
V_{g}^{\otimes} \sigma(\psi, \Psi)\left(V_{g}^{\otimes}\right)^{*}=\sigma\left(\psi \circ g^{-1}, \Psi\right) .
$$

where $U_{g}:=V_{g}^{\otimes}$ is the unique unitary operator such that $U_{g} \Upsilon=\Upsilon$ and, on each subspace of $\mathcal{F}_{+}(\mathcal{H})$ with $n$ particles coincides to the corresponding tensor product of $n$ copies of $V_{g}$. Since $W(\psi)=e^{-i \sigma(\psi, \Psi) \text {, (46) }}$ implies

$$
U_{g} W(\psi) U_{g}^{*}=\alpha_{g}(W(\psi)) .
$$

By linearity and continuity, such an identity extends to the whole Weyl algebra. Therefore we have found the wanted (and unique) unitary representation $U$ of $G_{\Im^{-}}$that implements $\alpha$ and leaves $\Upsilon$ fixed, and it 
is evident per construction that and $U$ coincides with the tensorialisation of $U \uparrow_{\mathcal{H}}$.

(c) Consider the subgroup of $G_{\Im^{-}}$definite by the elements $(R, 0,0), R \in S O(3)$. We have

$$
\left(U_{(R, 0,0)}\left\lceil_{\mathcal{H}} \varphi\right)(k, s):=\varphi\left(k, R^{-1}(s)\right) \text { for all } \varphi \in \mathcal{H} .\right.
$$

With the same procedure used in standard quantum mechanics, one sees that the invariant and irreducible subspaces of $\mathcal{H}$ under the representation $S O(3) \ni R \mapsto U_{(R, 0,0)}\lceil\mathcal{H}$ are labelled by the naturals $l=$ $0,1,2, \ldots$ and have the form $\mathcal{H}_{l}:=\left\{\sum_{m=-l}^{l} f_{m} Y_{l m} \mid f_{m} \in L^{2}\left(\mathbb{R}^{+}, 2 k d k\right)\right\}$, where $Y_{l m}(\theta, \phi)$ are the standard spherical harmonics. Therefore, the invariant subspaces of all $U_{(R, a, b)}$, if any, must be subspaces of some $\mathcal{H}_{l}$. Now consider $\chi(k)=e^{-k} \cdot \chi Y_{l m} \in \mathcal{H}_{l}$ by construction. However, using the fact that the $Y_{l m}$ are polynomials of exponentials in the variables $e^{i m \phi}$ and $\cos \theta$, one sees that

$$
(k, \theta, \phi) \mapsto e^{\cos \phi} \chi\left(e^{\cos \phi} k\right) Y_{l m}(\theta, \phi)=e^{-\exp (\cos \phi) k+\cos \phi} Y_{l m}(\theta, \phi)
$$

cannot belong to $\mathcal{H}_{l}$ and to any other analogous subspace. Therfore every $\mathcal{H}_{l}$ is not invariant under some of the elements $U_{(0, a, 0)}$ and thus $\mathcal{H}$ does not admit proper invariant subspaces with respect to the whole representation $G_{\Im^{-}} \ni(R, a, b) \mapsto U_{(R, a, b)}$ and it is, by consequence, irreducible.

A second important result concerns the positive-energy/uniqueness properties of $\lambda$. In Minkowski QFT positivity of energy, i.e. positivity of the spectrum of the self-adjoint generator of the unitary representation of Minkowskian time displacements, is a stability requirement. It guarantees that, under small (external) perturbations, the system does not collapse to lower and lower energy states. In general spacetimes the notion of energy is associated with a notion of Killing time. This interpretation can be extended to this case too, namely to the theory on $\Im^{-}$(later we shall come back to that in $M$ ). The positive-energy requirement is fulfilled for the "asymptotic" notion of time associated with limit values $\widetilde{Y}$ towards $\Im^{-}$ of a timelike future-directed vector field $Y$ in $M$, when $\widetilde{Y} \in \mathfrak{g}_{\Im^{-}}$. Notice that $Y$ may not be a Killing vector outside $\Im^{-}$; it is enough that $Y \rightarrow \widetilde{Y} \in \mathfrak{g}_{\Im^{-}}$. This includes the case $Y=X$ in particular, due to Proposition 3.1

In the following, $\{\exp \{t Z\}\}_{t \in \mathbb{R}}$ is the one-parameter subgroup of $G_{\Im^{-}}$generated by any $Z \in \mathfrak{g}_{\Im^{-}}$and $\left\{\alpha_{t}^{(Z)}\right\}_{t \in \mathbb{R}}$ is the associated one-parameter group of $*$-automorphisms of $\mathcal{W}\left(\Im^{-}\right)$(35).

Proposition 4.2. $\quad$ Consider an expanding universe with cosmological horizon $(M, g, X, \Omega, \gamma)$, the state $\lambda$ on $\Im^{-}$defined in (36) and a timelike future-directed vector field $Y$ in $M$ such that $Y \rightarrow \widetilde{Y} \in \mathfrak{g}_{\Im^{-}}$ pointwisely approaching $\Im^{-}(Y=X$ in particular, in view of Proposition 3.1). The following holds.

(a) The unitary group $\left\{U_{t}^{(\widetilde{Y})}\right\}_{t \in \mathbb{R}}$ which implements $\alpha^{(\widetilde{Y})}$ leaving fixed the cyclic GNS vector in the GNS representation of $\lambda$ is strongly continuous with nonnegative self-adjoint generator $H^{(\widetilde{Y})}=-\left.i \frac{d}{d t} t_{s}(\widetilde{Y})\right|_{t=0}$. (b) The restriction of $H^{(\widetilde{Y})}$ to the one-particle space has no zero modes if and only if $\widetilde{Y}$ vanishes on a zero-measure subset of $\Im^{-}$.

Proof. From Proposition 3.4 one has that $\tilde{Y}(\ell, s)=f(s) \partial_{\ell}$ for some non negative smooth function $f: \mathbb{S}^{2} \rightarrow \mathbb{R}$. Therefore $\exp \{t \widehat{Y}\}$ amounts to the displacement $(\ell, s) \rightarrow(\ell+f(s) t, s)$. As a consequence of (43), the one parameter group $\alpha^{(\widetilde{Y})}$ is unitarily represented by $\left\{U_{t}^{(\widetilde{Y})}\right\}_{t \in \mathbb{R}} \cdot U_{t}^{(\widetilde{Y})}$ is the tensorialisation (as in (41)) of the (representation of the) unitary group in the one-particle space $V_{t}: \mathcal{H} \rightarrow \mathcal{H}$, with

$$
\left(V_{t} \phi\right)(k, s)=e^{i t k f(s)} \psi(k, s)=\left(e^{i t h^{(\tilde{Y})}} \psi\right)(k, s), \quad \text { for all } \phi \in \mathcal{H} .
$$


From standard theorems of operator theory one obtains that $\mathbb{R} \ni t \mapsto V_{t}$ is strongly continuous with selfadjoint generator $h^{(\widetilde{Y})}$, in the one-particle space $\mathcal{H}=L^{2}\left(\mathbb{R}^{+} \times \mathbb{S}^{2} ; 2 k d k \wedge \epsilon_{\mathbb{S}^{2}}\right)$, given by $\left(h^{(\widetilde{Y})} \phi\right)(k, s)=$ $k f(s) \phi(k, s)$, defined in the dense domains $\mathcal{D}\left(h^{(\widetilde{Y})}\right)$ made of the elements of the Hilbert space $L^{2}\left(\mathbb{R}^{+} \times\right.$ $\left.\mathbb{S}^{2} ; 2 k d k \wedge \epsilon_{\mathbb{S}^{2}}\right)$ such that the right-hand side belongs to $L^{2}\left(\mathbb{R}^{+} \times \mathbb{S}^{2} ; 2 k d k \wedge \epsilon_{\mathbb{S}^{2}}\right)$. It is so evident that, since $f \geq 0$, for every $\psi \in \mathcal{D}(H)$

$$
\left\langle\phi, h^{(\widetilde{Y})} \phi\right\rangle=\int_{0}^{+\infty} 2 k d k \int_{\mathbb{S}^{2}} \epsilon_{\mathbb{S}^{2}}(s)|\phi(k, s)|^{2} k f(s) \geq 0,
$$

and thus $\sigma\left(h^{(\widetilde{Y})}\right) \subset[0,+\infty)$. Passing to the whole Fock space by (41) the result remains unchanged for the whole generator $H^{(\widetilde{Y})}=0+h^{(\widetilde{Y})} \oplus I \otimes h^{(\widetilde{Y})} \oplus h^{(\widetilde{Y})} \otimes I \oplus \cdots$ using standard properties of generators. The last statement is a trivial consequence of (48) using $\widetilde{Y}=f \partial_{\ell}$.

The result applies in particular for $\widetilde{Y}=\partial_{\ell}$, since it is always possible to view $\partial_{\ell}$ as the limit value of some timelike vector field of $M$. For expanding universes with cosmological horizon as in Theorem 2.1, if $X:=-\gamma \partial_{\tau}$, then $X \rightarrow \partial_{\ell}$ approaching $\Im^{-}$. In this above case the energy-positivity property applies for $X$ and there are no zero modes.

This is not the whole story, since the positive-energy property for $\partial_{\ell}$, determine completely $\lambda$.

Theorem 4.2. Consider the state $\lambda$ on $\Im^{-}$defined in (36) and its GNS representation. The following holds.

(a) The state $\lambda$ defined in (36) is the unique pure quasifree state on $\mathcal{W}\left(\Im^{-}\right)$satisfying both:

(i) it is invariant under $\alpha^{\left(\partial_{\ell}\right)}$,

(ii) the unitary group which implements $\alpha^{\left(\partial_{\ell}\right)}$ leaving fixed the cyclic GNS vector is strongly continuous with nonnegative self-adjoint generator (energy positivity condition).

(b) Let $\omega$ be a pure (not necessarily quasifree) state on $\mathcal{W}\left(\Im^{-}\right)$which is $G_{\Im^{-}}$-invariant or, more weakly, $\alpha^{\left(\partial_{\ell}\right)}$-invariant. $\omega$ is the unique state on $\mathcal{W}\left(\Im^{-}\right)$satisfying both:

(i) it is invariant under $\alpha^{\left(\partial_{\ell}\right)}$,

(ii) it belongs to the folium of $\omega$.

Proof. The proofs of (a) and (b) are quite complicated, however they are identical to the proofs of the corresponding statements in Theorem 3.1 in Mo06] in the case where $\mathcal{F}$ is a Bondi frame. This is due to the fact that the self-adjoint generator of the unitary group $t \mapsto U_{t}$ implementing $\left\{\alpha_{t}^{\left(\partial_{\ell}\right)}\right\}_{t \in \mathbb{R}}$ and leaving $\Upsilon$ invariant is the tensorialisation of the positive self-adjoint generator $H$ acting in the one-particle space $L^{2}\left(\mathbb{R}^{+} \times \mathbb{S}^{2} ; 2 k d k \wedge \epsilon_{\mathbb{S}^{2}}\right)$, is $(H \widehat{\psi})(k, \theta, \phi)=k \widehat{\psi}(k, \theta, \phi)$. Note that $H$ is defined in the dense domains of the elements of the Hilbert space $L^{2}\left(\mathbb{R}^{+} \times \mathbb{S}^{2} ; 2 k d k \wedge \epsilon_{\mathbb{S}^{2}}\right)$ such that the right-hand side is still in $L^{2}\left(\mathbb{R}^{+} \times \mathbb{S}^{2} ; 2 k d k \wedge \epsilon_{\mathbb{S}^{2}}\right)$. It is so evident that $\sigma(H)=\sigma_{c}(H)=[0,+\infty)$.

The action of the one-parameter subgroup $\mathbb{R} \ni t \mapsto g^{\left(\partial_{\ell}\right)}(t)$ of $G_{\Im^{-}}$on fields defined on $\Im^{-}$coincides exactly with the one-parameter subgroup of the $B M S$ group on fields defined on $\Im^{+}$. Furthermore also the unitary representations of $G_{\Im^{-}}$and of the BMS group are identical when restricted to those subgroups. (Notice that in Mo06 the coordinate $\ell$ was indicated by $u$ and complex coordinates $\zeta=$ $e^{i \phi} \cot \theta / 2, \bar{\zeta}=e^{-i \phi} \cot \theta / 2$ were used on $\mathbb{S}^{2}$; finally, a different, but unitarily-equivalent, one-particle Hilbert space representation was used, exploiting the measure $d k$ instead of $2 k d k$.)

4.4. Interplay of QFT in $M$ and $Q F T$ on $\Im^{-}$. We have just seen that, in expanding universes $(M, g, \Omega, X, \gamma)$ with cosmological horizons $\Im^{-}$, the $C^{*}$-algebra of Weyl observable on $\Im^{-}$admits a preferred state $\lambda$ that is invariant under the action of $G_{\Im^{-}}$and enjoys some uniqueness properties. Since $G_{\Im^{-}}$includes physically relevant symmetries of the bulk $M$, we wonder whether or not it is possible to 
induce a state $\lambda_{M}$ on the bulk starting from $\lambda$. If it were be possible, we would expect that $\lambda_{M}$ would enjoy some invariance properties with respect to the (possible) isometries individuated by Killing vectors which preserve $\Im^{-}$. To consider this issue, here we concentrate on algebraic properties beforehand, establishing the existence of a nice interplay between $\mathcal{W}\left(\Im^{-}\right)$and $\mathcal{W}(M)$ under suitable hypotheses on the considered symplectic forms. That interplay will be used to define $\lambda_{M}$ in the next subsection.

The symplectic form $\sigma_{M}$ on $\mathcal{S}(M)$ defined in (30) can be equivalently rewritten as the integral of a 3 -form,

$$
\sigma_{M}\left(\varphi_{1}, \varphi_{2}\right):=\int_{S} \chi\left(\varphi_{1}, \varphi_{2}\right)
$$

where, the Cauchy surface $S$ is future oriented and, in any local coordinate patch:

$$
\chi\left(\varphi_{1}, \varphi_{2}\right):=-\frac{1}{6}\left(\varphi_{2} \nabla^{\mu} \varphi_{1}-\varphi_{1} \nabla^{\mu} \varphi_{2}\right) \sqrt{-\widehat{g}} \epsilon_{\mu \alpha \beta \gamma} d x^{\alpha} \wedge d x^{\beta} \wedge d x^{\gamma},
$$

Above $\epsilon_{\mu \alpha \beta \gamma}$ is the totally antisymmetric Levi Civita symbol. As it is known $\sigma_{M}\left(\varphi_{1}, \varphi_{2}\right)$ does not depend on the considered Cauchy surface. As soon as $S$ is moved back in the past, it seems it tends to coincide with $\Im^{-}$. Actually this is not necessarily the case, since $\Im^{-}$and Cauchy surfaces in $M$ may have different topologies. In particular, information could get lost through the time-like past infinity $i^{-}$, the tip of the cone representing $\Im^{-}$. That point does not belong to $\widehat{M}$ in our hypotheses. However one may expect that, in certain cases at least, assuming that each $\varphi_{i}$ extends to $\Gamma \varphi_{i} \in \mathrm{S}\left(\Im^{-}\right)$smoothly, it holds

$$
\sigma_{M}\left(\varphi_{1}, \varphi_{2}\right)=\int_{\Im^{-}} \chi\left(\Gamma \varphi_{1}, \Gamma \varphi_{2}\right)
$$

Now, by direct inspection one verifies that, for $\psi_{1}, \psi_{2} \in \mathbf{S}\left(\Im^{-}\right)$,

$$
\int_{\Im^{-}} \chi\left(\psi_{1}, \psi_{2}\right)=\gamma^{2} \int_{\mathbb{R} \times \mathbb{S}^{2}}\left(\psi_{2} \frac{\partial \psi_{1}}{\partial \ell}-\psi_{1} \frac{\partial \psi_{2}}{\partial \ell}\right) d \ell \wedge \epsilon_{\mathbb{S}^{2}}(\theta, \phi),
$$

where $\gamma$ is the last constant in $(M, g, \Omega, X, \gamma)$. Following this way one is led to expect that

$$
\sigma_{M}\left(\varphi_{1}, \varphi_{2}\right)=\sigma\left(\gamma \Gamma \varphi_{1}, \gamma \Gamma \varphi_{2}\right)
$$

Notice that this result is by no means trivial and it may not hold, since it strictly depends on the behaviour of the solutions of Klein-Gordon equations across $\Im^{-}$. In particular, as we have said above, information could get lost through the time-like past infinity $i^{-}$, the tip of the cone representing $\Im^{-}$. That point does not belong to $\widehat{M}$ in our hypotheses. In Mo06 it has been established that, for asymptotically flat spacetimes at future null infinity, whenever it is possible complete $\Im^{+}$with the tip $i^{+}$(and under suitable further hypotheses), an identity analogous to (53) is valid. In that case, however, the fact that the field was massless and conformally coupled played a crucial role. The extent is very different now, where the mass is present and the coupling with $R$ may not be conformal. Moreover, in our case the physical spacetime is $(M, g)$, whereas in the approach of [DMP06, Mo06, Mo07, Da07, it would be $\left(M, g / \Omega^{2}\right)$. Here we investigate on the consequences of such a validity of (53). In that case, the existence of $\Gamma$ : $\mathcal{S}(M) \rightarrow \mathrm{S}\left(\Im^{-}\right)$fulfilling (53) implies the existence of a isometric $*$-homomorphism from $\imath: \mathcal{W}(M) \rightarrow$ $\mathcal{W}\left(\Im^{-}\right)$. In this way the field observables of the bulk are mapped into observables of the theory on $\Im^{-}$. Moreover, the state $\lambda$ on $\Im^{-}$induces a preferred state $\lambda_{M}$ on $\mathcal{W}(M)$ via pull-back. This state enjoys interesting invariance properties with respect to the symmetries of $(M, g)$ which preserve $\Im^{-}$, as well as a positivity property with respect to timelike Killing vectors of $M$ which preserve $\Im^{-}$. 
Theorem 4.3. Consider an expanding universe with cosmological horizon $(M, g, X, \Omega, \gamma)$ and suppose that every $\varphi \in \mathcal{S}(M)$ extends smoothly to some $\Gamma \phi \in \mathrm{S}\left(\Im^{-}\right)$in order that (53) holds true:

$$
\sigma_{M}\left(\varphi_{1}, \varphi_{2}\right)=\sigma\left(\gamma \Gamma \varphi_{1}, \gamma \Gamma \varphi_{2}\right), \quad \text { for every } \varphi_{1}, \varphi_{2} \in \mathcal{S}(M) .
$$

In these hypotheses, there is an (isometric) *-homomorphism $\imath: \mathcal{W}(M) \rightarrow \mathcal{W}\left(\Im^{-}\right)$that identifies the Weyl $C^{*}$-algebra of the bulk $M$ with a sub $C^{*}$-algebra of the boundary $\Im^{-}$; it is completely determined by the requirement:

$$
\imath\left(W_{M}(\varphi)\right):=W(\gamma \Gamma \varphi), \quad \text { for all } \varphi \in \mathcal{W}(M) .
$$

Proof. Notice that the linear map $\gamma \Gamma: \mathcal{S}(M) \rightarrow \mathrm{S}\left(\Im^{-}\right)$has to be injective due to nondegenerateness of the symplectic form $\sigma$ and (53). Consider the sub Weyl- $C^{*}$-algebra $A_{M}$ of $\mathcal{W}\left(\Im^{-}\right)$generated by the elements $W(\gamma \Gamma \varphi)$ with $\varphi \in \mathcal{S}(M)$. Since Weyl $C^{*}$-algebras are determined up to (isometric) $*$-algebra isomorphisms, $A_{M}$ is nothing but the Weyl $C^{*}$-algebra associated with the symplectic space $(\gamma \Gamma(\mathcal{S}(M)), \sigma)$ and the map $\gamma \Gamma: \mathcal{S}(M) \rightarrow \Gamma(\mathcal{S}(M))$ is an isomorphism of symplectic spaces. Under these hypotheses BR022, there is a unique (isometric) $*$-isomorphism $\imath: \mathcal{W}(M) \rightarrow A_{M} \subset \mathcal{W}\left(\Im^{-}\right)$completely individuated by (54).

4.5. The preferred invariant state $\lambda_{M}$. To carry on our analysis, we show that, in the hypotheses of Theorem 4.3, a preferred state $\lambda_{M}$ on $\mathcal{W}(M)$ is induced by $\lambda$. That state enjoys very remarkable physical properties.

From now on, if $Y$ is a complete Killing vector of $(M, g)$, the associated one-parameter group of $g$ isometries, $\{\exp \{t Y\}\}_{t \in \mathbb{R}}$, has a natural action on $\mathcal{W}(M)$ obtained in this way. Since each $\exp \{t Y\}$ : $M \rightarrow M$ is an isometry, its pull-back action on fields preserves the symplectic form $\sigma_{M}$. As a consequence BR022, BGP96, there is a unique isometric $*$-isomorphism $\beta_{t}^{(Y)}: \mathcal{W}(M) \rightarrow \mathcal{W}(M)$ induced by the requirement that the identity holds

$$
\beta_{t}^{(Y)}\left(W_{M}(\varphi)\right):=W_{M}(\varphi \circ \exp \{-t Y\}), \quad \text { for every } \varphi \in \mathcal{S}(M) .
$$

In the following we shall call $\beta^{(Y)}:=\left\{\beta_{t}^{(Y)}\right\}_{t \in \mathbb{R}}$ the natural $*$-isomorphism action of $\{\exp \{t Y\}\}_{t \in \mathbb{R}}$ on $\mathcal{W}(M)$. Similarly, every $Z \in \mathfrak{g}_{\Im^{-}}$has a natural action $\alpha^{(Z)}$ on $\mathcal{W}\left(\Im^{-}\right)$in terms of isometric *-isomorphism, obtained by requiring,

$$
\alpha_{t}^{(Z)}(W(\psi)):=W(\psi \circ \exp \{-t Z\}), \quad \text { for every } \psi \in \mathrm{S}\left(\Im^{-}\right),
$$

since the pull-back action of the one-parameter group $\{\exp \{t Z\}\}_{t \in \mathbb{R}}$, generated by $Z$ on fields of $\mathrm{S}\left(\Im^{-}\right)$ preserves the symplectic form $\sigma$.

To stress a further important point, let us consider an expanding universe with cosmological horizon $(M, g, X, \Omega, \gamma)$ and let us suppose that every $\varphi \in \mathcal{S}(M)$ extends smoothly to some $\Gamma \phi \in \mathrm{S}\left(\Im^{-}\right)$in order that (53) holds true. In this case there is a uniquely defined smooth function $\widehat{\phi}$ defined on $M \cup \Im^{-}$, that reduces to $\phi$ in $M$ and to $\Gamma \phi$ on $\Im^{-}$. If $Y$ is a complete Killing vector of $(M, g)$ preserving $\Im^{-}$, the one parameter group generated by its unique extension $\widehat{Y}$ to $M \cup \Im^{-}$(Proposition 3.2 and Theorem 3.1) acts on $\widehat{\phi}$ globally. Taking the relevant restrictions of scalar fields and Killing vector fields we obtains:

$$
(\Gamma \phi) \circ \exp \{t \widetilde{Y}\}=\Gamma(\phi \circ \exp \{t Y\})
$$

where, as usual, $\widetilde{Y}:=\widehat{Y} \uparrow_{\Im^{-}}$. This identity holds true for every complete Killing vector $Y$ of $(M, g)$ that preserves $\Im^{-}$, for every $\phi \in \mathcal{S}(M)$ and for every $t \in \mathbb{R}$. As a straightforward consequence it holds

$$
\imath\left(\beta_{t}^{(Y)}(a)\right)=\alpha_{t}^{(\widetilde{Y})}(\imath(a)), \quad \text { for all } a \in \mathcal{W}(M) \text { and } t \in \mathbb{R} .
$$


Such an identity has an important consequence stated in the following theorem.

Theorem 4.4. Consider an expanding universe with cosmological horizon $(M, g, X, \Omega, \gamma)$ fulfilling the hypotheses of Theorem 4.3. Let $\lambda_{M}: \mathcal{W}(M) \rightarrow \mathbb{C}$ be the state induced by $\lambda$ defined in (36) through the isometric $*$-homomorphism $\imath$ (54):

$$
\lambda_{M}(a):=\lambda(\imath(a)), \text { for all } a \in \mathcal{W}(M) .
$$

$\lambda_{M}$ enjoys the following properties:

(a) Let $Y$ be some complete Killing vector field of $(M, g)$ which preserves $\Im^{-}$(if any), and let $\beta^{(Y)}$ the natural action on $\mathcal{W}(M)$, then $\lambda_{M}$ is invariant under $\beta^{(Y)}$ and the unitary one-parameter group $\left\{U_{t}^{(Y)}\right\}_{t \in \mathbb{R}}$, which implements $\beta^{(Y)}$ in the GNS representation of $\lambda_{M}$ leaving fixed the cyclic vector, is strongly continuous.

(b) If $Y$ above is everywhere timelike and future-directed in $M$, then

(i) the one-parameter group $\left\{U_{t}^{(Y)}\right\}_{t \in \mathbb{R}}$ has positive self-adjoint generator,

(ii) that generator has no zero-modes in the one-particle subspace, if $\tilde{Y}$ vanishes on a zero-measure subset of $\Im^{-}$.

Proof. As before, from now on, $\left(\mathcal{F}_{+}(\mathcal{H}), \Pi, \Upsilon\right)$ is the GNS triple of $\lambda$. First of all we notice that $\lambda_{M}$ is in fact a well-defined state on $\mathcal{W}(M)$ since $\imath$ is a $*$-homomorphism. $\lambda_{M}$ is quasifree associated with a real scalar product $\mu_{M}: \mathcal{S}(M) \times \mathcal{S}(M) \rightarrow \mathbb{R}$ defined as $\mu_{M}\left(\varphi, \varphi^{\prime}\right):=\mu\left(\gamma \Gamma \varphi, \gamma \Gamma \varphi^{\prime}\right)$. From this fact, it follows that the GNS triple of $\lambda_{M}$ can be constructed as $\left(\mathcal{F}_{+}\left(\mathcal{H}_{M}\right),\left.\Pi\right|_{A_{M}}, \Upsilon\right)$, where $A_{M} \subset \mathcal{W}\left(\Im^{-}\right)$is the sub $C^{*}$-algebra isomorphic to $\mathcal{W}(M)$ in view of Theorem 4.3, $\mathcal{H}_{M}$ is the Hilbert subspace of $\mathcal{H}$ given by the closure of the space of complex linear combinations of $K_{\mu}(\Gamma(\varphi))$, for every $\varphi \in \mathcal{S}(M)$ and, thus, $\mathcal{F}_{+}\left(\mathcal{H}_{M}\right)$ is a Fock subspace of $\mathcal{F}_{+}(\mathcal{H})$. In particular, the canonic $\mathbb{R}$-linear map $K_{\mu_{M}}: \mathcal{S}(M) \rightarrow \mathcal{H}_{M}$ is nothing but $K_{\mu_{M}}=K_{\mu} \circ \gamma \Gamma$.

(a) By construction, using the definition of $\lambda_{M}$, taking advantage of (56), and of the invariance of $\lambda$ as in (a) of Theorem 4.1, if $a \in \mathcal{W}(M)$, one has

$$
\lambda_{M}\left(\beta_{t}^{(Y)}(a)\right)=\lambda\left(\imath\left(\beta_{t}^{(Y)}(a)\right)\right)=\lambda\left(\alpha_{t}^{(\widetilde{Y})} \imath(a)\right)=\lambda(\imath(a))=\lambda_{M}(a) .
$$

This proves the first part of (a). To conclude the proof of (a), let $V_{t}^{(\widetilde{Y})}: \mathcal{H} \rightarrow \mathcal{H}$ the one-parameter group of unitaries that implements $\alpha_{t}^{(\widetilde{Y})}$ in the one-particle space $\mathcal{H}$ for $\lambda$. From $K_{\mu_{M}}=K_{\mu} \circ \gamma \Gamma$, (56) and (44) one has:

$$
\begin{aligned}
V_{t}^{(\widetilde{Y})} K_{\mu_{M}} \varphi=V_{t}^{(\widetilde{Y})} K_{\mu} \gamma \Gamma(\varphi) & =K_{\mu}((\gamma \Gamma \varphi) \circ \exp \{-t \widetilde{Y}\})=K_{\mu}(\gamma \Gamma(\varphi \circ \exp \{-t Y\})) \\
& =K_{\mu_{M}}(\varphi \circ \exp \{-t Y\}) .
\end{aligned}
$$

We have found that, for every $\varphi \in \mathcal{S}(M), V_{t}^{(\widetilde{Y})} K_{\mu_{M}} \varphi=K_{\mu_{M}}(\varphi \circ \exp \{-t Y\})$, and this immediately implies either that $V_{t}^{(\widetilde{Y})}$ leaves the one particle space of $\lambda_{M}, \mathcal{H}_{M}$, invariant either that $\left.V_{t}^{(\widetilde{Y})}\right|_{\mathcal{H}_{M}}$ implements $\beta_{t}^{(Y)}$ in $\mathcal{H}_{M}$. As a consequence of the structure of the GNS triple of $\lambda_{M}$, if $U_{t}^{(\widetilde{Y})}$ implements $\beta_{t}^{(\widetilde{Y})}$ unitarily in $\mathfrak{H}=\mathcal{F}_{+}(\mathcal{H})$ leaving $\Upsilon$ invariant, it leaves also invariant the structure of the GNS-Fock space of $\lambda_{M}$ and, therein, $U_{t}^{(\widetilde{Y})} \uparrow_{\mathcal{F}_{+}\left(\mathcal{H}_{M}\right)}$ implements $\alpha_{t}^{(Y)}$ unitarily in $\mathfrak{H}_{M}=\mathcal{F}_{+}\left(\mathcal{H}_{M}\right)$ leaving the cyclic vector invariant. In other words

$$
U_{t}^{(Y)}=U_{t}^{(\widetilde{Y})} \uparrow_{\mathcal{F}_{+}\left(\mathcal{H}_{M}\right)}
$$


Notice that $\mathbb{R} \ni \mapsto U_{t}^{(\widetilde{Y})} \Gamma_{\mathcal{F}_{+}\left(\mathcal{H}_{M}\right)}$ is strongly continuous since $\mathbb{R} \ni \mapsto U_{t}^{(\widetilde{Y})}$ is such. Moreover the selfadjoint generator of $U_{t}^{(\widetilde{Y})} \uparrow_{\mathcal{F}_{+}\left(\mathcal{H}_{M}\right)}$ is obtained by restricting that of $U_{t}^{(\widetilde{Y})} \uparrow_{\mathcal{F}_{+}\left(\mathcal{H}_{M}\right)}$ to $\mathcal{F}_{+}\left(\mathcal{H}_{M}\right)$. If the former generator is positive, the latter has to be so. In the considered case, the former is positive since $Y$ is timelike and future directed and thus we can apply (a) of Proposition 4.2. The same argument shows that the self-adjoint generator of $V_{t}^{(\widetilde{Y})} \Gamma_{\mathcal{H}_{M}}$ has no-zero modes if $V_{t}^{(\widetilde{Y})} \uparrow_{\mathcal{H}_{M}}$ has no-zero modes. This last fact happens if $\widetilde{Y}$ vanishes on a zero-measure subset of $\Im^{-}$due to (b) of Proposition 4.2,

Remark 4.2. As noticed before Proposition 4.2 positivity of energy is a stability requirement; it guarantees that, under small (external) perturbations, the system does not collapse to lower and lower energy states. The notion of energy is related, in curved spacetime, to the presence of a timelike Killing vector. The statement (b) of the theorem assures in particular that, in the presence of such a Killing vector, if it preserves $\Im^{-}$, the condition of energy positivity holds true. In the absence of any timelike Killing vector Proposition 4.2 assures anyway the validity of a positivity-energy condition, in particular with respect to the conformal Killing vector $X$. It is worth noticing that $X$ becomes a true $\widehat{g}$-Killing vector exactly on $\Im^{-}$by definition.

4.6. Testing the construction for the de Sitter case and for other FRW metrics. We proceed to show that the hypotheses of Theorem 4.3 are valid when $(M, g, X, \Omega, \gamma)$ is in the class of the FRW metrics considered in Theorem 2.1, so that the preferred state $\lambda_{M}$ exists for those spacetimes. That class includes the expanding region of de Sitter spacetime (see [BMG94, BM96] for a related analysisin the framework of Wightman's axioms). We shall verify, in this last case, that the preferred state $\lambda_{M}$ is nothing but the well-known de Sitter Euclidean vacuum or Buch-Davies state, $\omega_{E}$ SS76, BD78, Al85. Let us start with de Sitter space and review, very quickly, how $\omega_{E}$ is defined on $\mathcal{W}(M)$ when $(M, g)$ is the expanding region of de Sitter spacetime (actually there are infinite many such regions, each obtained form any other by the action an element of the subgroup of isometries of de Sitter metric preserving temporal-orientation ). The expanding region in de Sitter spacetime is the manifold

$$
M \simeq(-\infty, 0) \times \mathbb{R}^{3}, \quad g=a^{2}(\tau)\left[-d \tau \otimes d \tau+d r \otimes d r+r^{2} d \mathbb{S}^{2}(\theta, \varphi)\right],
$$

where $\tau \in(-\infty, 0)$ and where $r, \theta, \phi$ are standard spherical coordinates on $\mathbb{R}^{3}, a(\tau)=\gamma / \tau$ for some constant $\gamma<0$, so that and $R=12 / \gamma^{2}$. A class of, generally complex, solutions $\Phi_{\mathbf{k}}, \mathbf{k} \in \mathbb{R}^{3}$ of the Klein-Gordon equation $-\nabla_{a} \nabla^{a} \varphi+m^{2} \varphi+\xi R \varphi=0$ used to define $\omega_{E}$ is obtained introducing

$$
\Phi_{\mathbf{k}}(\tau, \mathbf{x}):=\frac{e^{i \mathbf{k} \cdot \mathbf{x}}}{(2 \pi)^{3 / 2}} \frac{\chi_{\mathbf{k}}(\tau)}{a(\tau)}
$$

where, $\mathbf{x} \in \mathbb{R}^{3}$ and the functions $\chi_{\mathbf{k}}$ are solutions of

$$
\frac{d^{2}}{d \tau^{2}} \chi_{\mathbf{k}}(\tau)+k^{2} \chi_{\mathbf{k}}(\tau)+a(\tau)^{2}\left[m^{2}+(\xi-1 / 6) R(\tau)\right] \chi_{\mathbf{k}}(\tau)=0
$$

We focus on the class of solutions (58) found in [SS76] :

$$
\chi_{\mathbf{k}}(\tau):=\frac{1}{2} \sqrt{-\pi \tau} e^{i \pi \nu / 2} \overline{H_{\nu}^{(2)}(-k \tau)}, \quad \text { where } \quad \nu:=\sqrt{\frac{9}{4}-12\left(m^{2} R^{-1}+\xi\right)}
$$

where $k:=|\mathbf{k}|, H_{\nu}^{(2)}$ is the second-type Hankel function. The sign in front of the square root in the definition of $\nu$ (which may be imaginary) does not affect the right-hand side of (60) and it could be 
fixed arbitrarily (either for $\nu$ real or imaginary). With those choices one finds the time-independent normalisation (time-independence follows from (59) immediately)

$$
\frac{d \overline{\chi_{\mathbf{k}}(\tau)}}{d \tau} \chi_{\mathbf{k}}(\tau)-\overline{\chi_{\mathbf{k}}(\tau)} \frac{d \chi_{\mathbf{k}}(\tau)}{d \tau}=i, \quad \text { for all } \tau \in(-\infty, 0)
$$

Remark 4.3. Actually, in [SS76, BD78, the contracting region in de Sitter spacetime was considered. There, $a(\tau)=\gamma / \tau$, for $\gamma>0$ and $\tau \in(0,+\infty)$. The appropriate modes are in that case 5 :

$$
\chi_{\mathbf{k}}(\tau):=\frac{1}{2} \sqrt{\pi \tau} e^{i \pi \nu / 2} H_{\nu}^{(2)}(k \tau), \quad \text { where } \quad \nu:=\sqrt{\frac{9}{4}-12\left(m^{2} R^{-1}+\xi\right)}
$$

the adaptation to our situation is straightforward, since (59) is invariant under $\tau \rightarrow-\tau$ and under complex conjugation. The complex conjugation in the modes (60) is necessary to obtain the asymptotic behaviour $\sim(2 k)^{-1 / 2} e^{-i k \tau}$ for $k \rightarrow+\infty, \tau \rightarrow-\infty$ and the correct normalisation (61).

Let us now show how $\omega_{E}$ is defined with the standard procedure of extraction of the positive frequency part of real wavefunctions, after having decomposed them with respect to the family of modes (58). To this end, take any (real by definition) $\varphi \in \mathcal{S}(M)$ and fix a Cauchy surface $\Sigma_{\tau}$ in $(M, g)$, individuated by the points in $M$ with the fixed value of $\tau$. As a last step define

$$
\widetilde{\varphi}(\mathbf{k}):=-i \int_{\mathbb{R}^{3}}\left[\frac{\partial \overline{\Phi_{\mathbf{k}}(\tau, \mathbf{x})}}{\partial \tau} \varphi(\tau, \mathbf{x})-\overline{\Phi_{\mathbf{k}}(\tau, \mathbf{x})} \frac{\partial \varphi(\tau, \mathbf{x})}{\partial \tau}\right] a(\tau)^{2} d \mathbf{x} .
$$

The right-hand side of (63) does not depend on the choice of $\tau$, as it follows per direct inspection exploiting both (59) and the Klein-Gordon equation. Let us remember either that $\varphi \in \mathcal{S}(M)$, either that its Cauchy data are real, smooth and compactly supported. Furthermore, taking into account that the the Hankel functions $H_{\nu}^{(2)}(z)$ decays as $z^{-1 / 2}$ as $|z| \rightarrow \infty$, it turns out that $\widetilde{\varphi} \in C^{\infty}\left(\mathbb{R}^{3} \backslash\{0\}\right)$ and it vanishes for $|\mathbf{k}| \rightarrow \infty$ faster than every power $|\mathbf{k}|^{-n}, n=1,2, \ldots$. From the known behaviour of the functions $H_{\nu}^{(2)}(z)$ in a neighbourhood of $z=0$ GR95, one sees that the leading divergence as $\mathbf{k} \rightarrow 0$ due to the functions $\chi_{\mathbf{k}}$ is of order $|\mathbf{k}|^{-|R e \nu|}$. This singularity does not affect the integrability of $|\widetilde{\varphi}|^{2}$ with respect to $d \mathbf{k}$ when $|R e \nu|<3 / 2$. We remark that integrability of $|\widetilde{\varphi}|^{2}$ entails integrability of $|\widetilde{\varphi}|$ in this case, since $\widetilde{\varphi}(\mathbf{k})$ vanish very fast for large $\mathbf{k}$. Eventually notice that, from the definition of $\nu,|R e \nu|<3 / 2$ is equivalent to require $m^{2}+\xi R>0$. Once one constructs $\widetilde{\varphi}$ out of ([63), the associated $\varphi$ can be obtained back as the pre-announced decomposition in terms of the modes $\Phi_{\mathrm{k}}$ :

$$
\varphi(\tau, \mathbf{x})=\int_{\mathbb{R}^{3}}\left[\Phi_{\mathbf{k}}(\tau, \mathbf{x}) \widetilde{\varphi}(\mathbf{k})+\overline{\Phi_{\mathbf{k}}(\tau, \mathbf{x}) \widetilde{\varphi}(\mathbf{k})}\right] d \mathbf{k} .
$$

This is a trivial consequence of (58), (61), (63), and of the standard properties of F Fourier transform for smooth compactly supported functions on $\mathbb{R}^{3}$.

As we have said above, in the hypotheses $m^{2}+\xi R>0$, one has $\widetilde{\varphi} \in L^{2}\left(\mathbb{R}^{3} ; d \mathbf{k}\right) \cap L^{1}\left(\mathbb{R}^{3} ; d \mathbf{k}\right)$ for every $\varphi \in \mathcal{S}(M)$. In this case, per direct computation one verifies that, if $\varphi_{1}, \varphi_{2} \in \mathcal{S}(M)$,

$$
-2 \operatorname{Im}\left\{\int_{\mathbb{R}^{3}} \widetilde{\widetilde{\varphi}_{1}}(\mathbf{k}) \widetilde{\varphi}_{2}(\mathbf{k}) d \mathbf{k}\right\}=\int_{\mathbb{R}^{3}}\left(\varphi_{2} \partial_{\tau} \varphi_{1}-\varphi_{1} \partial_{\tau} \varphi_{2}\right) a^{2}(\tau) d \mathbf{x}=: \sigma_{M}\left(\varphi_{1}, \varphi_{2}\right) .
$$

\footnotetext{
${ }^{5}$ The form of the modes as presented in BD78 BD82 is different due to the absence of the overall exponential exp $-i \pi \nu / 2$, which would affect the final results and the normalisation (61) for $\nu$ imaginary. Nevertheless, the final form of the two-points function presented in BD78, BD82] is not affected by this new factor. Therefore, in all cases it coincides with that found in [SS76] previously.
} 
The (restriction to $M$ of the) Euclidean vacuum in de Sitter space is nothing but the quasifree state $\omega_{E}$ on $\mathcal{W}(M)$ completely identified by

$$
\omega_{E}\left(W_{M}(\varphi)\right)=e^{-\frac{1}{2} \int_{\mathbb{R}^{3}} \overline{\widetilde{\varphi}(\mathbf{k})} \widetilde{\varphi}(\mathbf{k}) d \mathbf{k}}, \quad \text { for every } \varphi \in \mathcal{S}(M) .
$$

Notice that the constraint (93) is automatically fulfilled in view of (65)).

Remark 4.4. (1) The maximally extended de Sitter spacetime can be realized by glueing together two isometric spacetimes - one expanding and the other contracting, when moving towards the future - on the common cosmological horizon. The obtained spacetime is maximally symmetric and admits $S O(1,5)$ as group of isometries. The state $\omega_{E}$ extends to a globally defined state on the whole complete de Sitter spacetime Al85.

(2) The extended state $\omega_{E}$ (which coincides with that constructed in terms of the modes (62) in the contracting region) is invariant under the whole group of isometries, $O(1,5)$, of the maximally extended de Sitter spacetime, so that it is also fixed under symmetries which do not preserve the horizon.

(3) Among the class of $S O(1,5)$-invariant $\alpha$-vacua individuated by Allen [Al85, $\omega_{E}$ is the only globally Hadamard state (the two-point function of the remaining ones has singularities also for non null related arguments).

Theorem 4.5. Consider the expanding universe $(M, g, X, \Omega, \gamma)$ given by the expanding portion of de Sitter space, so that

$$
M \simeq(-\infty, 0) \times \mathbb{R}^{3}, \quad g=a^{2}(\tau)\left[-d \tau \otimes d \tau+d r \otimes d r+r^{2} d \mathbb{S}^{2}(\theta, \varphi)\right],
$$

where $\tau \in(-\infty, 0)$ and where $r, \theta, \phi$ are the standard spherical coordinates on $\mathbb{R}^{3}$, whereas $X=\partial_{\tau}$ and $\Omega(\tau)=a(\tau)=\gamma / \tau$, for some constant $\gamma<0$ so that $R=12 / \gamma^{2}$. Consider a quantum scalar KleinGordon field propagating in $(M, g)$ with Klein-Gordon operator $-\nabla_{a} \nabla^{a}+m^{2}+\xi R$ where $m^{2}+\xi R>0$ so that $\omega_{E}$ can be defined. The following holds.

(a) If $m^{2}+\xi R>\frac{5}{48} R$ (though this requirement may be dropped as discussed in Remark 4.5), every $\varphi \in \mathcal{S}(M)$ extends smoothly to some $\Gamma \phi \in \mathrm{S}\left(\Im^{-}\right)$and (53) holds true.

(b) With the same hypotheses, the preferred state $\lambda_{M}$ on $\mathcal{S}(M)$ coincides with the restriction to $M$ of the Euclidean vacuum $\omega_{E}$.

Proof. (a) Consider a wavefunction $\varphi \in \mathcal{S}(M)$. As it is well-known it satisfies $\varphi=E f$ where $E$ : $C_{0}^{\infty}(M) \rightarrow \mathcal{S}(M)$ is the causal propagator and $f$ is some real smooth and compactly supported function in $M$. Since the maximally extended de Sitter spacetime $M^{\prime}$ is globally hyperbolic and $M \subset M^{\prime},-$ so that $C_{0}^{\infty}(M) \subset C_{0}^{\infty}\left(M^{\prime}\right)$ - one can focus on the wavefunction $\varphi^{\prime}:=E^{\prime} f$, where $E^{\prime}$ is the causal propagator in $M^{\prime}$. By construction $\left.\varphi^{\prime}\right|_{M}=\varphi$, so that $\varphi^{\prime}$ is a smooth extension of $\varphi$. Since $\Im^{-} \subset M^{\prime}$, all that implies that $\varphi$ extends to $\Im^{-}$smoothly (and uniquely) and this extension is $\lim _{\rightarrow \Im^{-}} \varphi=\varphi^{\prime} \uparrow_{\Im^{-}}$. In this way, an $\mathbb{R}$-linear map $\Gamma: \mathcal{S}(M) \ni \varphi \rightarrow \varphi^{\prime} \Upsilon_{\Im^{-}} \in C_{0}^{\infty}\left(\Im^{-}\right)$is defined. To conclude (a), it is enough to prove either that $\operatorname{Ran} \Gamma \subset \mathrm{S}\left(\Im^{-}\right)$either that $\Gamma$ preserves the symplectic forms. Let us prove them. Since, for large $z$ (with $|\arg z| \leq \pi-\epsilon$ )

$$
H_{\nu}^{(2)}(z)=\sqrt{\frac{2}{\pi z}} e^{-i\left(z-\frac{\pi \nu}{2}-\frac{\pi}{4}\right)}\left[1+O\left(\frac{1}{z}\right)\right],
$$

making use of (58) and (60), the identity (64) can be recast as

$$
\varphi(\tau, \mathbf{x})=\frac{e^{-i \frac{\pi}{4}}}{\gamma 4 \pi^{3 / 2}} \int_{\mathbb{S}^{2}} \epsilon_{\mathbb{S}^{2}}(\theta, \phi) \int_{0}^{+\infty} d k k e^{i\left(k r \cos \lambda_{\mathbf{x}}(\theta, \phi)-k \tau\right)}\left[\tau+O\left(\frac{1}{k}\right)\right] \sqrt{k} \widetilde{\varphi}(k, \theta, \phi)+c . c .,
$$


where $\lambda_{\mathbf{x}}(\theta, \phi) \in[0, \pi]$ is the angle between $\mathbf{x}$ and $\mathbf{k}$ and + c.c. stands for the adding to the result of the integration of its the complex conjugation. The iterated integrations make sense and can be interchanged (via Fubini-Tonelli theorem) since the functions $(k, \theta, \phi) \mapsto k \sqrt{k} \widetilde{\varphi}(k, \theta, \phi)$ and $(k, \theta, \phi) \mapsto \sqrt{k} \widetilde{\varphi}(k, \theta, \phi)$ are integrable in the measure $d \mathbf{k}$, as it arises from the analysis performed above. They are smooth everywhere but $\mathbf{k}=0$, they vanish very fast at large $|\mathbf{k}|$ and the divergence for $\mathbf{k}=0$ of $\widetilde{\varphi}$ is that of $1 /|\mathbf{k}|^{-R e|\nu|}$, with our weaker requirement on $\nu, R e|\nu|<3 / 2$ (i.e. $m^{2}+\xi R>0$ ) for $\nu$ as in (60). Now we remind that (see the proof of Theorem 2.1) $\tau=(u+v) / 2$ and $r=(u-v) / 2$, and we have to take the limit $v \rightarrow-\infty$ to achieve $\Im^{-}$. The contribution due to the factor of $O\left(\frac{1}{k}\right)$ vanishes due to the Riemann-Lebesgue lemma (followed by Lebesgue dominate convergence theorem to handle the external integration) and, interchanging again the integrations, it results in:

$$
(\Gamma \varphi)\left(u, \theta_{\mathbf{x}}, \phi_{\mathbf{x}}\right)=\lim _{s \rightarrow+\infty} \frac{e^{-i \frac{\pi}{4}}}{\gamma 4 \pi^{3 / 2}} \int_{0}^{+\infty} d k \int_{\mathbb{S}^{2}} \epsilon_{\mathbb{S}^{2}}(\theta, \phi) \frac{k s}{2} e^{i \frac{k s}{2}\left[\cos \lambda_{\mathbf{x}}(\theta, \phi)+1\right]} e^{-i u k} \sqrt{k} \widetilde{\varphi}(k, \theta, \phi)+c . c .
$$

That limit can be computed using integration by parts exactly as in the appendix A2 of [DMP06]. Roughly speaking, the limit vanishes because of Riemann-Lebesgue lemma, except for $\cos \lambda_{\mathbf{x}}(\theta, \phi)+1=0$, so that the only contribution arises when the values $\theta, \phi$ of $\mathbf{k}$ coincides with the values of the corresponding angles of $\mathbf{- x}$. In practice, one rotates the axes so that the axis $z$ coincides with $\mathbf{x}$ and, thinking of $\widetilde{\varphi}$ as a function of $k, c, \phi$ where $c:=\cos \theta \in[-1,1]$, one re-arranges the expression above as

$$
(\Gamma \varphi)\left(u, \theta_{\mathbf{x}}, \phi_{\mathbf{x}}\right)=\lim _{s \rightarrow+\infty} \frac{-i e^{-i \frac{\pi}{4}}}{\gamma 4 \pi^{3 / 2}} \int_{0}^{+\infty} d k \int_{0}^{2 \pi} d \phi \int_{-1}^{1} d c \frac{\partial}{\partial c}\left(e^{i \frac{k s}{2}[c+1]}\right) e^{-i u k} \sqrt{k} \widetilde{\varphi}(k, c, \phi)+c . c .
$$

where $\theta_{\mathbf{x}}=0$ in our case. The right-hand side can be expanded using integration by parts and only the contribution for $c=-1$ (that is $\theta=-\pi$, i.e. $\mathbf{k} /|\mathbf{k}|=-\mathbf{x} /|\mathbf{x}|$ ) survives, the others vanish as $s \rightarrow+\infty$, due to Riemann-Lesbegue's lemma (interchanging various integrations using Fubini-Tonelli theorem and finally taking advantage of dominate convergence theorem). The integration over $\phi$ produces a trivial factor $2 \pi$ since the dependence from $\phi$ of the involved functions obviously disappears as $\theta=0, \pi$. The final result reads, using the initial generic choice for the axes $x, y, z$ :

$$
(\Gamma \varphi)\left(u, \theta_{\mathbf{x}}, \phi_{\mathbf{x}}\right)=\frac{i 2 \pi e^{-i \frac{\pi}{4}}}{\gamma 4 \pi^{3 / 2}} \int_{0}^{+\infty} d k e^{-i u k} \sqrt{k} \widetilde{\varphi}\left(k, \eta\left(\theta_{\mathbf{x}}, \phi_{\mathbf{x}}\right)\right)+c . c .,
$$

$\eta: \mathbb{S}^{2} \rightarrow \mathbb{S}^{2}$ denoting the parity inversion $\mathbb{S}^{2} \ni \mathbf{n} \mapsto-\mathbf{n} \in \mathbb{S}^{2}$. Dropping the index $\mathbf{x}$, and viewing $\theta, \phi$ as the standard coordinates on $\Im^{-}$, the obtained result can be re-written as

$$
(\gamma \Gamma \varphi)(\ell, \theta, \phi)=i \frac{e^{-i \frac{\pi}{4}}}{(-\gamma)} \int_{0}^{+\infty} d k \frac{e^{-i \ell k}}{\sqrt{2 \pi}} \sqrt{\frac{k}{2(-\gamma)}} \widetilde{\varphi}\left(\frac{k}{(-\gamma)}, \eta(\theta, \phi)\right)+c . c .
$$

where we have passed to the standard Bondi coordinates on $\Im^{-}$, i.e. $\ell, \theta, \phi$ with $u=-\gamma \ell$. In our hypotheses on $\varphi$ and $\nu$-it is precisely on this point that the stronger requirement on $\nu,|R e \nu|<1$, namely $m^{2}+\xi R>\frac{5}{48} R$, is used to deal with the possible divergence at $k=0$ - the functions $(k, \theta, \phi) \mapsto$ $\sqrt{\frac{k}{2}} \widetilde{\varphi}(k, \eta(\theta, \phi))$ and $(k, \theta, \phi) \mapsto k \sqrt{\frac{k}{2}} \widetilde{\varphi}(k, \eta(\theta, \phi))$ belong also to $L^{2}\left(\mathbb{R}^{+} \times \mathbb{S}^{2} ; d k \wedge \epsilon_{\mathbb{S}^{2}}(\theta, \phi)\right.$ ) (and so are the functions with $k$ rescaled as $\left.(-\gamma)^{2} k\right)$. This implies that both the functions $\Gamma \varphi, \partial_{\ell} \Gamma \varphi$ belong to $L^{2}\left(\mathbb{R} \times \mathbb{S}^{2} ; d \ell \wedge \epsilon_{\mathbb{S}^{2}}\right)$. In this way we have found that $\operatorname{Ran} \Gamma \subset \mathrm{S}\left(\Im^{-}\right)$. Actually we have obtained much more: by means of use of (37) and (39), (68) implies that

$$
\sigma\left(\gamma \Gamma \varphi, \gamma \Gamma \varphi^{\prime}\right)=-2 \operatorname{Im}\left\{(-\gamma)^{-2} \int_{\mathbb{R}^{+} \times \mathbb{S}^{2}} d k \wedge \epsilon_{\mathbb{S}^{2}} 2 k \frac{k}{2(-\gamma)} \overline{\widetilde{\varphi}} \overline{\left(\frac{k}{(-\gamma)}, \eta(\theta, \phi)\right)} \tilde{\varphi^{\prime}}\left(\frac{k}{(-\gamma)}, \eta(\theta, \phi)\right)\right\}
$$




$$
=-2 \operatorname{Im}\left\{\int_{\mathbb{R}^{+} \times \mathbb{S}^{2}} k^{2} d k \wedge \epsilon_{\mathbb{S}^{2}} \overline{\widetilde{\varphi}(k, \theta, \phi)} \widetilde{\varphi}^{\prime}(k, \theta, \phi)\right\}=-2 \operatorname{Im}\left\{\int_{\mathbb{R}^{3}} d \mathbf{k} \overline{\widetilde{\varphi}(\mathbf{k})} \widetilde{\varphi^{\prime}}(\mathbf{k})\right\}=\sigma_{M}\left(\varphi, \varphi^{\prime}\right),
$$

where in the last step we exploited (65). We have obtained that $\gamma \Gamma$ preserves the symplectic form as requested.

(b) Exactly as in the last step of the proof of (a), since the functions $(k, \theta, \phi) \mapsto \sqrt{\frac{k}{2}} \widetilde{\varphi}(k, \eta(\theta, \phi))$ and $(k, \theta, \phi) \mapsto k \sqrt{\frac{k}{2}} \widetilde{\varphi}(k, \eta(\theta, \phi))$ are also in $L^{2}\left(\mathbb{R}^{+} \times \mathbb{S}^{2} ; d k \wedge \epsilon_{\mathbb{S}^{2}}(\theta, \phi)\right)$, (40) and (68) imply:

$$
\begin{gathered}
\mu\left(K_{\lambda} \gamma \Gamma \varphi, K_{\lambda} \gamma \Gamma \varphi\right)=(-\gamma)^{-2} \int_{\mathbb{R}^{+} \times \mathbb{S}^{2}} d k \wedge \epsilon_{\mathbb{S}^{2}} 2 k \frac{k}{2(-\gamma)} \overline{\widetilde{\varphi}\left(\frac{k}{(-\gamma)}, \eta(\theta, \phi)\right)} \widetilde{\varphi}\left(\frac{k}{(-\gamma)}, \eta(\theta, \phi)\right) \\
=\int_{\mathbb{R}^{+} \times \mathbb{S}^{2}} k^{2} d k \wedge \epsilon_{\mathbb{S}^{2}} \overline{\widetilde{\varphi}(k, \theta, \phi)} \widetilde{\varphi}(k, \theta, \phi)=\int_{\mathbb{R}^{3}} d \mathbf{k} \widetilde{\widetilde{\varphi}(\mathbf{k})} \widetilde{\varphi}(\mathbf{k})
\end{gathered}
$$

Therefore, for every $\varphi \in \mathcal{S}(M)$, in view of (66),

$$
\lambda_{M}\left(W_{M}(\varphi)\right):=\lambda(W(\gamma \Gamma \varphi))=e^{-\mu\left(K_{\lambda} \gamma \Gamma \varphi, K_{\lambda} \gamma \Gamma \varphi\right) / 2}=e^{-\frac{1}{2} \int_{\mathbb{R}^{3}} \overline{\widetilde{\varphi}(\mathbf{k})} \widetilde{\varphi}(\mathbf{k}) d \mathbf{k}}=\omega_{E}\left(W_{M}(\varphi)\right),
$$

and this concludes the proof.

Remark 4.5. The requirement $m^{2}+\xi R>\frac{5}{48}$ R, i.e. $|R e \nu|<1$ is used above to assure that the smooth function $\Gamma \varphi$, obtained by taking the limit towards $\Im^{-}$of $\varphi \in \mathcal{S}(M)$, belongs to $\mathrm{S}\left(\Im^{-}\right)$. Actually the requirement can be dropped (preserving, however, the weaker one $m^{2}+\xi R>0$ ) by a slight change in the definition (30) of $\mathrm{S}\left(\Im^{-}\right)$. As an alternate, but technically more involved and "non local", way to build up all our construction, we could first define

$$
\mathrm{S}\left(\Im^{-}\right):=\left\{\left.\psi \in C^{\infty}\left(\mathbb{R} \times \mathbb{S}^{2}\right)\left|\int_{\mathbb{R} \times \mathbb{S}^{2}}\right| \widehat{\psi}(k, \theta, \phi)\right|^{2}|k| d k \wedge \epsilon_{\mathbb{S}^{2}}(\theta, \phi)<+\infty\right\}
$$

where $\widehat{\psi}$ indicates the Fourier-Plancherel transform of the Schwartz distribution $\psi$ (as discussed in the Appendix $\mathrm{C}$ of [Mo07]). Then the symplectic form on $\Im^{-}$could be defined as the right-hand side of (39). In this way, the identity (33) would hold true in a weaker limit sense, employing a suitable regularisation of $\psi_{1}$ and/or $\psi_{2}$ by means of sequences of smooth compactly supported functions. Then the construction either of the $G_{\Im^{-}}$-invariant state $\lambda$ on $\mathcal{W}\left(\Im^{-}\right)$either of its GNS triple together with the uniqueness/positive energy theorems would closely resemble to our previous analysis. With the modified definition of $\mathrm{S}\left(\Im^{-}\right)$above introduced, the smooth real function $\Gamma \varphi$, whose Fourier transform on $\Im^{-}$is $(k, \theta, \phi) \mapsto \sqrt{\frac{k}{2}} \widetilde{\varphi}(k, \eta(\theta, \phi))$, as seen in the proof above, would belong to $\mathrm{S}\left(\Im^{-}\right)$up to the only hypothesis $|R e \nu|<3 / 2$ (i.e. $m^{2}+\xi R>0$ ), since the divergence in $k=0$ of $\widetilde{\varphi}$ is of order $k^{-|R e \nu|}$.

To conclude we have the last promised theorem: The hypotheses of Theorem 4.3 are fulfilled, and thus $\lambda_{M}$ is defined, for FRW metrics as in Theorem 2.1, provided the mass $m$ of the Klein-Gordon field and/or the constant $\xi$ are large enough.

Theorem 4.6. Consider a quantum scalar Klein-Gordon field $\varphi$, satisfying the equation of motion $-\nabla_{a} \nabla^{a} \varphi+m^{2} \varphi+\xi R \varphi=0$ and propagating in an expanding universe $(M, g, X, \Omega, \gamma)$ as in the hypotheses of Theorem 2.1] and with $\ddot{a}(\tau)=2 \gamma / \tau^{3}+O\left(1 / \tau^{4}\right)$. (So that, in particular $R=12 / \gamma^{2}+O(1 / \tau)$

$$
M \simeq(-\infty, 0) \times \mathbb{R}^{3}, \quad g=a^{2}(\tau)\left[-d \tau \otimes d \tau+d r \otimes d r+r^{2} d \mathbb{S}^{2}(\theta, \varphi)\right],
$$


$\tau \in(-\infty, 0)$ and $r, \theta, \phi$ standard spherical coordinates on $\mathbb{R}^{3}, X=\partial_{\tau}$ and $\Omega=a(\tau)=\gamma / \tau+O\left(1 / \tau^{2}\right)($ as $\tau \rightarrow-\infty)$, for some constant $\gamma<0$.)

If $m^{2} \gamma^{2}+12 \xi>2$, every $\varphi \in \mathcal{S}(M)$ extends smoothly to some $\Gamma \phi \in \mathrm{S}\left(\Im^{-}\right)$and (53) holds true.

Proof. Here, we exploit the same notation, i.e. $\mathbf{x}, \mathbf{k}$, as in the proof of Theorem 4.5, In particular $\nu:=\sqrt{\frac{9}{4}-\left(m^{2} \gamma^{2}+12 \xi\right)}$, so that $\nu \geq 0$ when $\frac{9}{4}-\left(m^{2} \gamma^{2}+12 \xi\right) \geq 0$ in the following. However the sign of $\nu$ could be fixed arbitrarily (and this applies for imaginary $\nu$, in particular), since the functions we shall employ are invariant under $\nu \rightarrow-\nu$.

As a first step, we notice that if $\varphi \in \mathcal{S}(M)$, it extends to $\Im^{-}$smoothly so that $\Gamma \varphi:=\lim _{\rightarrow} \Im^{-} \varphi \in C^{\infty}\left(\Im^{-}\right)$ does exist. This is because, as found in the proof of Theorem 2.1, the spacetime $(M, g)$ extends to a larger spacetime equipped with a metric $\widehat{g}$ obtained by multiplying the metric of the closed static Einstein universe with a strictly positive smooth factor. Since closed static Einstein universe is globally hyperbolic and global hyperbolicity does not depend on nonsingular conformal rescaling of the metric, $(M, g)$ itself is included in a globally hyperbolic spacetime. With the same argument used for de Sitter spacetime in the proof of Theorem 4.5, one has that every $\varphi \in \mathcal{S}(M)$ extends to $\Im^{-}$smoothly. We have now to show that $\operatorname{Ran} \Gamma \subset \mathrm{S}\left(\Im^{-}\right)$and that $\Gamma$ preserves the symplectic forms.

First of all, analogously to what done in the de Sitter case, we determine a class of modes $\Psi_{\mathbf{k}}(\tau, \mathbf{x})$ that will be useful in decomposing the solutions of Klein-Gordon equation in order to take the limit of wavefunctions towards $\Im^{-}$.

$$
\Psi_{\mathbf{k}}(\tau, \mathbf{x}):=\frac{e^{i \mathbf{k} \cdot \mathbf{x}}}{(2 \pi)^{3 / 2}} \frac{\rho_{\mathbf{k}}(\tau)}{a(\tau)}
$$

where, taking the exponential factor into account, the Klein-Gordon equation reduces to the following equation for the functions $(-\infty, 0) \ni \tau \mapsto \psi_{\mathbf{k}}(\tau)$,

$$
\begin{aligned}
& \frac{d^{2}}{d \tau^{2}} \rho_{\mathbf{k}}(\tau)+\left(V_{0}(\mathbf{k}, \tau)+V(\tau)\right) \rho_{\mathbf{k}}(\tau)=0 \\
& \text { with } \quad V_{0}(\mathbf{k}, \tau):=k^{2}+\left(\frac{\gamma}{\tau}\right)^{2}\left[m^{2}+\left(\xi-\frac{2}{\gamma^{2}}\right)\right], \quad V(\tau)=O\left(1 / \tau^{3}\right)
\end{aligned}
$$

Comparing with Klein-Gordon equation, one sees that $V_{0}(\mathbf{k}, \tau)+V(\tau)=k^{2}+a(\tau)^{2}\left[m^{2}+(\xi-1 / 6) R(\tau)\right]$ where $V_{0}$ is nothing but the the contribution of pure de Sitter metric and $V$ is a perturbation. If we dropped the perturbation $V(\tau)$, the functions $\rho_{\mathbf{k}}$ would reduce to the functions $\chi_{\mathbf{k}}$ and the modes $\Psi_{\mathbf{k}}$ would reduce to the modes $\Phi_{k}$ used to construct $\omega_{E}$ beforehand; moreover (70) would reduce to (59). Notice that the curvature of the spacetime does not coincide with $12 / \gamma^{2}$ as in de Sitter spacetime, but it reads $R(\tau)=12 / \gamma^{2}+O(1 / \tau)$ and $a(\tau)=\gamma / \tau+O\left(1 / \tau^{2}\right)$. It follows that the added potential $V(\tau)=O\left(1 / \tau^{3}\right)$ above. A formal solution of (170) is obtained in terms of the series:

$$
\begin{gathered}
\rho_{\mathbf{k}}(\tau)=\chi_{\mathbf{k}}(\tau) \\
+(-1)^{n} \sum_{n=1}^{+\infty} \int_{-\infty}^{\tau} d t_{1} \int_{-\infty}^{t_{1}} d t_{2} \cdots \int_{-\infty}^{t_{n-1}} d t_{n} S_{\mathbf{k}}\left(\tau, t_{1}\right) S_{\mathbf{k}}\left(t_{1}, t_{2}\right) \cdots S_{\mathbf{k}}\left(t_{n-1}, t_{n}\right) V\left(t_{1}\right) V\left(t_{2}\right) \cdots V\left(t_{n}\right) \chi_{\mathbf{k}}\left(t_{n}\right)
\end{gathered}
$$

where we have used the antisymmetric solution of (59):

$$
S_{\mathbf{k}}\left(t, t^{\prime}\right):=-i\left(\overline{\chi_{\mathbf{k}}(t)} \chi_{\mathbf{k}}\left(t^{\prime}\right)-\overline{\chi_{\mathbf{k}}\left(t^{\prime}\right)} \chi_{\mathbf{k}}(t)\right), \quad t, t^{\prime} \in(-\infty, 0)
$$


satisfying, in view of antisymmetry and (61),

$$
S_{\mathbf{k}}(t, t)=0 \quad \text { and }\left.\quad \frac{\partial}{\partial t} S_{\mathbf{k}}\left(t, t^{\prime}\right)\right|_{t^{\prime}=t}=1 .
$$

By direct inspection and making use of (73), one sees that the right-hand side of (71) defines a solution of (70) if one is allowed to interchange the $\tau$-derivative operator - up to the second order - with the sign of sum. This is always possible when the series itself and the series of the derivatives of first and second order converge $\tau$-uniformly in a neighbourhood of every fixed $\tau \in(-\infty, 0)$. Actually the locally $\tau$-uniform convergence of the series of derivatives of second order directly follows from the uniform convergence of those of zero and first order, when one either takes (59) into account either refers to the solutions $\chi_{\mathbf{k}}$ and the solutions $S_{\mathbf{k}}$. Using the expression (60) of the modes $\chi_{\mathbf{k}}$, expanding $H_{\nu}^{(2)}$ in terms of Bessel functions $J_{ \pm \nu}$ GR95 and, finally, exploiting standard integral representations valid for $\operatorname{Re} \nu>-1 / 2$ (formula 5 in 8.411 in GR95) of $J_{\nu}$, one achieves the following bounds for $R e \nu<1 / 2$ (that is $m^{2} \gamma^{2}+12 \xi>2$ ), for $\tau<-1$, and for some constant $C_{\nu} \geq 0$

$$
\begin{aligned}
& \left|\chi_{\mathbf{k}}(\tau)\right| \leq C_{\nu}(-\tau)^{R e \nu+1 / 2}\left(k^{R e \nu}+k^{-R e \nu}\right) \\
& \left|\frac{d \chi_{\mathbf{k}}(\tau)}{d \tau}\right| \leq C_{\nu}(-\tau)^{R e \nu+1 / 2}\left(k^{R e \nu}+k^{-R e \nu}\right)(1+k),
\end{aligned}
$$

where $k=|\mathbf{k}|$. Furthermore, for the same reasons it is possible to obtain the following (non optimal) k-uniform bound for $\operatorname{Re} \nu<1 / 2$, for $t_{2} \leq t_{1}<-1$, and for some other constant $C_{\nu}^{\prime} \geq 0$

$$
\left|S_{\mathbf{k}}\left(t_{1}, t_{2}\right)\right| \leq C_{\nu}^{\prime}\left(t_{1} t_{2}\right)^{R e \nu+1 / 2} .
$$

Now fix any $T<-1$ and consider $\tau \in(-\infty, T]$, so that $|V(\tau)| \leq K_{T} /(-\tau)^{3}$, for some constant $K_{T} \geq 0$. From (74), one sees with a few of trivial computations, that the series in the right-hand side of (71) and that of the $\tau$-derivatives are $\tau$-uniformly dominated, respectively, by

$$
\left(k^{R e \nu}+k^{-R e \nu}\right) S_{\nu, T}, \quad\left(k^{R e \nu}+k^{-R e \nu}\right)(1+k) S_{\nu, T},
$$

where $S_{\nu, T}$ is the following convergent series of positive constants

$$
S_{\nu, T}:=C_{\nu} \sum_{n=1}^{+\infty}\left(\frac{2 C_{\nu}^{\prime} K_{T}}{1-2 R e \nu}\right)^{n} \frac{1}{n !} \frac{1}{\left((-T)^{1-2 R e \nu}\right)^{n-1 / 2}} .
$$

Summarising, we can conclude that (71) defines a solution of (70) and that, the same equation entails the solution to be smooth. As a straightforward consequence we also have the following $\tau$-uniform bound valid on $(-\infty, T]$

$$
\begin{aligned}
\left|\rho_{\mathbf{k}}(\tau)-\chi_{\mathbf{k}}(\tau)\right| & \leq\left(k^{R e \nu}+k^{-R e \nu}\right) S_{\nu, T}, \\
\left|\frac{d \rho_{\mathbf{k}}(\tau)}{d \tau}-\frac{d \chi_{\mathbf{k}}(\tau)}{d \tau}\right| & \leq 2\left(k^{R e \nu}+k^{-R e \nu}\right)(1+k) S_{\nu, T} .
\end{aligned}
$$

This implies that, at fixed $\tau$, the measurable (since limit of measurable functions) functions $\mathbb{R}^{3} \ni \mathbf{k} \mapsto$ $\rho_{\mathbf{k}}(\tau)$ and $\mathbb{R}^{3} \ni \mathbf{k} \mapsto \frac{d \rho_{\mathbf{k}}(\tau)}{d \tau}$ do not grow, for large $|\mathbf{k}|$, fast than $|\mathbf{k}|^{R e \nu}$ and $|\mathbf{k}|^{1+R e \nu}$ respectively. Moreover, their divergence at $\mathbf{k}=0$ cannot be worse than that of $\mathbb{R}^{3} \ni \mathbf{k} \mapsto \chi_{\mathbf{k}}(\tau)$ and $\mathbb{R}^{3} \ni \mathbf{k} \mapsto \frac{d \chi_{\mathbf{k}}(\tau)}{d \tau}$, that is $k^{-|R e \nu|}$.

Finally, notice that each term in the series in the right-hand side of (71) and in the analogy for $d \rho_{\mathbf{k}} / d \tau$ 
vanishes as $\tau \rightarrow-\infty$ by construction. In view of the fact that, $\tau$-uniformly, the series in (76) dominates either the series in the right-hand side of (71) either the series of $\tau$-derivatives, we are allowed to interchange the symbol of limit with that of sum, obtaining

$$
\lim _{\tau \rightarrow-\infty}\left(\rho_{\mathbf{k}}(\tau)-\chi_{\mathbf{k}}(\tau)\right)=0 \quad \text { and } \quad \lim _{\tau \rightarrow-\infty}\left(\frac{d \rho_{\mathbf{k}}(\tau)}{d \tau}-\frac{d \chi_{\mathbf{k}}(\tau)}{d \tau}\right)=0 .
$$

This result has a first important consequence. Using equation (70), one sees that the function $\tau \mapsto$ $\frac{d \overline{\rho_{\mathbf{k}}(\tau)}}{d \tau} \rho_{\mathbf{k}}(\tau)-\overline{\rho_{\mathbf{k}}(\tau)} \frac{d \rho_{\mathbf{k}}(\tau)}{d \tau}$ is actually a constant. The value of this constant can be computed by taking the limit as $\tau \rightarrow-\infty$, making use of (61), (78) and taking into account the fact that, for $\mathbf{k}$ fixed, $\frac{d \rho_{\mathbf{k}}(\tau)}{d \tau}$ and $\rho_{\mathbf{k}}(\tau)$ are bounded on $(-\infty, T]$ (notice that these functions have no limit for $\tau \rightarrow-\infty$ ), as one can show employing the asymptotic behaviour of $H_{\nu}^{(2)}(z)$ for large values of the argument $z$. In this way one finds

$$
\frac{d \overline{\rho_{\mathbf{k}}(\tau)}}{d \tau} \rho_{\mathbf{k}}(\tau)-\overline{\rho_{\mathbf{k}}(\tau)} \frac{d \rho_{\mathbf{k}}(\tau)}{d \tau}=i
$$

Now, to analyse the behaviour of $\Gamma \varphi$, we can follow the same way as that followed in de Sitter space. Take any (real by definition) $\varphi \in \mathcal{S}(M)$ and fix a Cauchy surface $\Sigma_{\tau}$ in $(M, g)$ individuated by the points in $M$ with the fixed value of $\tau$; eventually define

$$
\widetilde{\varphi}(\mathbf{k}):=-i \int_{\mathbb{R}^{3}}\left[\frac{\partial \overline{\Psi_{\mathbf{k}}(\tau, \mathbf{x})}}{\partial \tau} \varphi(\tau, \mathbf{x})-\overline{\Psi_{\mathbf{k}}(\tau, \mathbf{x})} \frac{\partial \varphi(\tau, \mathbf{x})}{\partial \tau}\right] a(\tau)^{2} d \mathbf{x} .
$$

The right-hand side of (80) does not depend on the choice of $\tau$, as it follows from direct inspection, exploiting (70). Remembering that $\varphi \in \mathcal{S}(M)$, so that its Cauchy data are real, smooth and compactly supported, we have that their Fourier transform are of Schwartz class. Afterwards, exploiting the fact that both the measurable functions $\mathbb{R}^{3} \ni \mathbf{k} \mapsto \rho_{\mathbf{k}}(\tau)$ and $\mathbb{R}^{3} \ni \mathbf{k} \mapsto \frac{d \rho_{\mathbf{k}}(\tau)}{d \tau}$ grows at most as a polynomial with degree two for large $|\mathbf{k}|$, and that their divergence at $\mathbf{k}=0$ is at most of order $k^{-|\operatorname{Re} \nu|}$ with $\operatorname{Re\nu }<1 / 2$, we find that $\widetilde{\varphi} \in C^{\infty}\left(\mathbb{R}^{3} \backslash\{0\}\right)$ and it vanishes for $|\mathbf{k}| \rightarrow \infty$ faster than every power $|\mathbf{k}|^{-n}, n=1,2, \ldots$. In particular $\widetilde{\varphi} \in L^{2}\left(\mathbb{R}^{3} ; d \mathbf{k}\right) \cap L^{1}\left(\mathbb{R}^{3} ; d \mathbf{k}\right)$. Once one knows $\widetilde{\varphi}$ by (80), the associated $\varphi$ can be constructed out of a decomposition in terms of modes $\Psi_{\mathbf{k}}$ :

$$
\varphi(\tau, \mathbf{x})=\int_{\mathbb{R}^{3}}\left[\Psi_{\mathbf{k}}(\tau, \mathbf{x}) \widetilde{\varphi}(\mathbf{k})+\overline{\Psi_{\mathbf{k}}(\tau, \mathbf{x}) \widetilde{\varphi}(\mathbf{k})}\right] d \mathbf{k} .
$$

This is a trivial consequence of (80), (69), (79), and of the standard properties for the Fourier transform of smooth compactly supported functions on $\mathbb{R}^{3}$. Eventually, per direct computation, one verifies that, if $\varphi_{1}, \varphi_{2} \in \mathcal{S}(M)$

$$
-2 \operatorname{Im}\left\{\int_{\mathbb{R}^{3}} \widetilde{\widetilde{\varphi}_{1}}(\mathbf{k}) \widetilde{\varphi}_{2}(\mathbf{k}) d \mathbf{k}\right\}=\int_{\mathbb{R}^{3}}\left(\varphi_{2} \partial_{\tau} \varphi_{1}-\varphi_{1} \partial_{\tau} \varphi_{2}\right) a^{2}(\tau) d \mathbf{x}=: \sigma_{M}\left(\varphi_{1}, \varphi_{2}\right) .
$$

We are now in position to draw some conclusions. Indeed, if $\varphi \in \mathcal{S}(M), p \in \Im^{-}$and $\left(\tau_{q}, \mathbf{x}_{q}\right)$ are the coordinates of $q \in M$, we can write down

$$
(\Gamma \varphi)(p)=\lim _{q \rightarrow p} \int_{\mathbb{R}^{3}} \frac{\mathbf{k}^{3}}{(2 \pi)^{3 / 2} \cdot \mathbf{x}_{q}}\left(\rho_{\mathbf{k}}\left(\tau_{q}\right)-\chi_{\mathbf{k}}\left(\tau_{q}\right)\right) \widetilde{\varphi}(\mathbf{k})+\lim _{q \rightarrow p} \int_{\mathbb{R}^{3}} \mathbf{k} \frac{e^{i \mathbf{k} \cdot \mathbf{x}_{q}}}{(2 \pi)^{3 / 2}} \chi_{\mathbf{k}}(\tau) \widetilde{\varphi}(\mathbf{k})+c . c .
$$


As $q \rightarrow p \in \Im^{-}, \tau_{q} \rightarrow-\infty$ so that $\left(\rho_{\mathbf{k}}\left(\tau_{q}\right)-\chi_{\mathbf{k}}\left(\tau_{q}\right)\right) \rightarrow 0$ due to (78). Moreover, since (76) is valid, we have the $\tau$-uniform bound

$$
\left|\frac{e^{i \mathbf{k} \cdot \mathbf{x}}}{(2 \pi)^{3 / 2}}\left(\rho_{\mathbf{k}}(\tau)-\chi_{\mathbf{k}}(\tau)\right) \widetilde{\varphi}(\mathbf{k})\right| \leq \frac{S_{\nu, T}}{(2 \pi)^{3 / 2}}\left(|\mathbf{k}|^{R e \nu}+|\mathbf{k}|^{-R e \nu}\right)|\widetilde{\varphi}(\mathbf{k})|
$$

where the right hand side is integrable because $\operatorname{Re} \nu<1 / 2, \widetilde{\varphi} \in L^{1}\left(\mathbb{R}^{3} ; d \mathbf{k}\right) \cap L^{2}\left(\mathbb{R}^{3} ; d \mathbf{k}\right)$ and it vanishes faster than any power for $|\mathbf{k}| \rightarrow+\infty$. Lesbegue's dominate convergence theorem implies that the former limit in (83) vanishes. The remaining limit has been computed in the proof of (a) in Theorem 4.5. The final result reads as follows: if $(\ell, \theta, \phi)$ are Bondi coordinates of $p \in \Im^{-}$and $\eta: \mathbb{S}^{2} \rightarrow \mathbb{S}^{2}$ is the inversion $\mathbf{n} \mapsto-\mathbf{n}$ on the sphere,

$$
(\gamma \Gamma \varphi)(\ell, \theta, \phi)=i \frac{e^{-i \frac{\pi}{4}}}{(-\gamma)} \int_{0}^{+\infty} d k \frac{e^{-i \ell k}}{\sqrt{2 \pi}} \sqrt{\frac{k}{2(-\gamma)}} \widetilde{\varphi}\left(\frac{k}{(-\gamma)}, \eta(\theta, \phi)\right)+c . c .
$$

From this point on the proof carries on up to the conclusions exactly as in the proof of (a) in Theorem 4.5, since (65) holds also in our generalised case, as (82) shows.

Remark 4.6. (1) Theorem 4.6 is also valid relaxing the hypothesis to the case $\xi=1 / 6$ and $m=0$. In that case the proof is similar to that of the case studied in [DMP06, Mo06].

(2) The validity of Hadamard property for the states $\lambda_{M}$ will be investigated in a forthcoming paper. However, a first scrutiny shows that it does hold for the states $\lambda_{M}$ considered in Theorem 4.6 provided the two-point function of such a state is a distribution of $\mathcal{D}^{\prime}(M \times M)$. The proof being almost the same as that preformed in Mo07. The distributional requirement is fulfilled if the functions $\Gamma \varphi, \varphi \in \mathcal{S}(M)$, satisfy a suitable decay property as $\ell \rightarrow-\infty$.

\section{Conclusions and open issues.}

In this manuscript, the initial purpose was to show that, at a geometrical level, it exists a strong interplay between a certain class of Friedmann-Robertson-Walker spacetimes and asymptotically flat spacetimes. More precisely, imposing some suitable constraints on the expansion factor $a(t)$, we were able to prove in theorem 2.1 that the FRW background can be extended to a larger spacetime which is a past conformal completion of an asymptotically flat spacetime $\widehat{M}$ with metric $\widehat{g}$. Therefore, as a non trivial byproduct of this statement, it turns out that the cosmological horizon shares many similar geometric properties of past (or future) null infinity.

Such an interplay is later generalized in definition 3.1 where we introduce a novel notion of an expanding universe with geodesically complete cosmological particle horizon. It is worth to stress again that, while in Penrose compactification, only the conformal factor - $\Omega$ - plays a key role, on the opposite in the set of backgrounds we are taking into account, it enters the fray a further new ingredient. This is a future oriented timelike vector $X$ which is a conformal Killing vector for the metric $\widehat{g}$; its interplays with $\Omega$ are of great relevance in our construction since, as a byproduct, they allows us to determine explicilty the structure of the isometry group of the horizon.

Concerning this specific topic, we find the first significant difference from the standard asymptotically flat scenario where the symmetry group is the so-called Bondi-Metzner-Sachs group, whereas, here we have the iterated semidirect product $S O(3) \ltimes\left(C^{\infty}\left(\mathbb{S}^{2}\right) \ltimes C^{\infty}\left(\mathbb{S}^{2}\right)\right)$. Such a result suggests us that, while the overall scenario we are dealing with does not completely overlap with the asymptotically flat scenario, 
nonetheless, it is still conceivable to readapt in this framework some of the properties of a scalar quantum field theory as discussed in DMP06, Mo06, Mo07.

Such a task was undertaken in section 4 , where we considered a generic massive scalar Klein-Gordon equation with an arbitrary coupling to curvature (hence a more generic scenario than the one envisaged in DMP06]). Under the assumption that each solution of such an equation for compactely supported intial data projects on the horizon to a rapidly decreasing smooth function - say $\psi$ - and that such a projection preserves a suitable symplectic form, then we were able to draw some interesting conclusions:

- the projection map between classical fields extends also at a level of Weyl algebras, namely we can embed the bulk Weyl $C^{*}$-algebra as a $C^{*}$-subalgebra of the horizon counterpart,

- we can select for the theory on the horizon a preferred state $\lambda$ which is quasi-free and pure. Furthermore $\lambda$ is the unique state which, besides the previous properties, is also invariant under the action of the horizon symmetry group. Moreover, for any future oriented timelike vector field $Y$ in the bulk such that it projects on the horizon to $\widetilde{Y}, i$. e. a generator of the isometry algebra, then the unitary group of operators implementing the action of $\widetilde{Y}$ on the GNS representation of $\lambda$ is strongly continuous with a non negative self-adjoint generator,

- the embedding between Weyl algebras can be exploited in order to pull-back $\lambda$ to a state $\lambda_{M}$ in the bulk which is still quasi-free and invariant under the action of any isometry for the bulk metric which preserves the cosmological horizon. Furthermore, whenever the Killing vector is everywhere future oriented and timelike, than the one-parameter group of operators implementing such an action is positive and self-adjoint.

As previously mentioned these results hold true under certain hypotheses which we tested in section 4.6 where we studied the behaviour of solutions for the Klein-Gordon equation of motion with an arbitrary coupling to curvature either in the de-Sitter either in the FRW background. Our analysis shows - see theorem 4.6 - that the hypotheses made at the beginning of section 4 , hold true at least whenever certain conditions between the relevant parameters in the equation of motion are satisfied.

On the overall we feel safe to claim that the analysis we performed proves that the investigation of a quantum field theory in a suitable cosmological background by means of an horizon counterpart is a viable option. Hence, as a future perspective, we feel worth to carry on the investigation started in this manuscript and, in particular we envisage to address some key questions. Besides enquiring if it is possible to extend the domain of applicability of theorem 4.6, we wish to further discuss the properties for the bulk state. In particular our long-term aim is to prove either that $\lambda_{M}$ is pure either that it is Hadamard (so that it can be used in renormalization procedures, especially for the stress energy tensor Wa94, Mo03, HW05]) and, furthermore we should also investigate possible relations with the adiabatic states often exploited in the study of field theories on FRW backgrounds [JS02, LR90, O107, Pa69. Concerning the validity of Hadamard property, it holds true for $\lambda_{M}$ when $M$ is deSitter spacetime (in this case $\lambda_{M}$ is the Euclidean vacuum), however, a first scrutiny shows that it does hold for all the states $\lambda_{M}$ considered in Theorem 4.6 provided the two-point function of such a state is a distribution of $\mathcal{D}^{\prime}(M \times M)$. The proof being almost the same as that preformed in Mo07.

From a more broader perspective, it is fair to admit that the results in DMP06] on the boundary theory where, to a certain, extent, more complete since we were able to interpret the projection on null infinity of each solution of the wave equation conformally coupled to curvature as a BMS invariant field. We would like to recover a similar statement in the scenario we are dealing with. Alas such a task cannot leave aside from a full-fledged study of all possible unitary and irreducibles representations of the horizon symmetry group. Since the latter is, by direct inspection, an infinite dimensional group, this is manifestly a daunting task which will require a paper on its own. 
Eventually, at last but not at least, it would be interesting to extend our results to interacting fields. From a physical perspective this would be the most appealing scenario since, as mentioned in the introduction, nowadays cosmological models are often based upon a single scalar field whose dynamic is governed by a non trivial potential. It could also be worth to investigate possible applications of our results to the description of dark matter. Being weakly interacting, it is feasible to model it, at least in a first approximation, as a free quantum scalar field on a curved background. Although here we do not address all the above mentioned topics, we feel like that this manuscript could be a nice first step towards this direction and we hope to discuss many if not all these mentioned points in a forthcoming manuscript.

\section{Acknowledgements.}

The work of C.D. is supported by the von Humboldt Foundation and that of N.P. has been supported by the German DFG Research Program SFB 676. We would like to thank K. Fredenhagen and R. Brunetti for useful discussions.

\section{A Proof of some technical results.}

Technical comments on Definition 2.1. From the requirement 2, $R_{a b}=0$ holds in a neighbourhood of $\Im^{+}$. So it is guaranteed that:

$$
\Omega R_{a b} \rightarrow 0 \quad \text { smoothly approaching } \Im^{+} .
$$

Eq. (85), smoothness of both $\widehat{R}_{a b}$ and $\Omega$ on $\Im^{+}$, and $\Omega \uparrow_{\Im^{+}}=0$, using the formula Wa84

$$
R_{a b}=\widehat{R}_{a b}+2 \Omega^{-1} \widehat{\nabla}_{a} \widehat{\nabla}_{b} \Omega+\widehat{g}_{a b} \widehat{g}^{c d}\left(\Omega^{-1} \widehat{\nabla}_{c} \widehat{\nabla}_{d} \Omega-3 \Omega^{-2} \widehat{\nabla}_{c} \Omega \widehat{\nabla}_{d} \Omega\right)
$$

imply that $\widehat{g}^{c d} \widehat{\nabla}_{c} \Omega \widehat{\nabla}_{d} \Omega=0$ on $\Im^{+}$that is $\widehat{g}(d \Omega, d \Omega)=0$ thereon. As a consequence - (a) - $\Im^{+}$is a null surface and - (b) - the contravariant representation of $d \Omega, n^{a}:=\widehat{\nabla}^{a} \Omega$ is null and tangent to $\Im^{+}$. Using notations as in the definition, $\omega \Omega=: \Omega^{\prime}, \omega^{2} \widehat{g}=: \widehat{g}^{\prime}$. Equation (85) and (86) implies that, if $\omega$ is chosen in order to fulfil $\widehat{\nabla}_{a}\left(\omega^{4} n^{a}\right)=0$ (and this is always possible because it can be interpreted as ordinary differential equation on every integral line of $n$ Wa84), one finds

$$
\widehat{\nabla}_{a}^{\prime} \widehat{\nabla}_{b}^{\prime} \Omega^{\prime} \Im_{\Im^{+}}=0
$$

where the covariant derivatives are referred to the metric $\widehat{g}^{\prime}$. (En passant, it implies that $\widehat{\Gamma}_{a b}^{\prime \Omega}=0$ on $\Im^{+}$, so that $\Im^{-}$is totally geodesic with respect to the connection induced on $\Im^{+}$by the $\widehat{g}^{\prime}$.) Finally (87) implies immediately that - (c) -the integral lines of the vector $\hat{\nabla}^{\prime b} \Omega^{\prime}$ are geodesics of the metric $\widehat{g}^{\prime}$, they are null-like because $\widehat{\nabla}^{\prime b} \Omega^{\prime}=\omega^{-1} n^{b}$ on $\Im^{+}$.

By direct inspection, one verifies that (87) implies (and hence is equivalent to) $\hat{\nabla}_{a}\left(\omega^{4} n^{a}\right)=0$ taking (85) and (86) into account. (851) and (86) imply also that $\hat{\nabla}^{\prime b} \widehat{\nabla}_{b}^{\prime} \Omega^{\prime} \uparrow_{\Im^{+}}=2 \Omega^{-1} \hat{\nabla}_{b}^{\prime} \Omega^{\prime} \hat{\nabla}^{\prime b} \Omega \uparrow_{\Im^{+}}$, which, inserted in (86)) and after multiplication by $\Omega$, proves that (87) is also equivalent to $\widehat{\nabla}^{\prime b} \widehat{\nabla}_{b}^{\prime} \Omega^{\prime} \uparrow_{\varsigma^{+}}=0$.

Proof of Proposition 3.1. (a) If there were a smooth extension of $X$ to $\bar{M}$ it would be unique by continuity, moreover, by continuity again, it would define a Killing vector for $\widehat{g}$ when restricting to the surface $\Im^{-}$, because the right-hand side of (21) vanishes there. We, in fact, will prove the existence of 
a smooth extension to the whole $\widehat{M}$. Coordinates $(\ell, \Omega, \theta, \phi)$ are defined in a neighbourhood $U \subset \widehat{M}$ of $\Im^{-}=\partial M$. Using the whole class of smooth curves $\gamma: t \rightarrow\left(\ell_{0}, t, \theta_{0}, \phi_{0}\right)$ where $\left(\ell_{0}, \theta_{0}, \phi_{0}\right) \in \mathbb{R} \times \mathbb{S}^{2}$ are fixed arbitrarily, and the transport equations Ge77, Hal04]

$$
\begin{array}{cc}
\dot{\gamma}^{a} \widehat{\nabla}_{a} \widehat{X}_{b}=\dot{\gamma}^{a}\left(\widehat{F}_{a b}+\frac{1}{2} \widehat{g}_{a b} \widehat{\varphi}\right), & \dot{\gamma}^{a} \widehat{\nabla}_{a} \widehat{\varphi}=\dot{\gamma}^{a} \widehat{K}_{a} \\
\dot{\gamma}^{a} \widehat{\nabla}_{a} \widehat{F}_{b c}=\dot{\gamma}^{a}\left(\widehat{R}_{b c a d} \widehat{X}^{d}+\widehat{K}_{[b} \widehat{g}_{c] a}\right), & \dot{\gamma}^{a} \widehat{\nabla}_{a} \widehat{K}_{b}=\dot{\gamma}^{a}\left(\widehat{X}^{d} \widehat{\nabla}_{d} \widehat{L}_{a b}+\widehat{\varphi} \widehat{L}_{a b}+2 \widehat{R}_{d(a} \widehat{F}_{b)}{ }^{d}\right)
\end{array}
$$

(where $\widehat{L}_{a b}:=\widehat{R}_{a b}-\frac{1}{6} \widehat{g}_{a b} \widehat{R}$ ) we can "transport" $X, F_{a b}=\widehat{\nabla}_{a} X_{b}-\widehat{\nabla}_{b} X_{a}, \varphi \widehat{g}:=\frac{1}{2} \mathscr{L}_{X}(\widehat{g})$, and $K_{a}:=\widehat{\nabla}_{a} \varphi$ beyond $\Im^{-}$in $U$. The transported fields $\widehat{X}, \widehat{F}, \widehat{\varphi}$, and $\widehat{K}_{a}$ are nothing but the solutions of the first order differential equations (88), with initial conditions given by the known fields $X, F, \varphi, K$ evaluated on a fixed smooth surface $\Omega=\Omega(\ell, \theta, \phi)$ completely included in $M \cap U$. In $M, \widehat{X}$ coincides with $X$ itself (and $\widehat{F}$ coincides with $F$ itself and so on), since every conformal Killing vector field fulfils transport equations (88) Ge77, Hal04 and uniqueness theorem holds for solutions of ordinary differential equations. Outside $M$ one gets a smooth field $\widehat{X}$ anyway, due to the jointly dependence of solution of differential equations from the initial data (assigned on a smooth surface as well ). Obviously the constructed field $\widehat{X}$ does not need to fulfil conformal Killing equations outside $\bar{M}$. In this way we have constructed a smooth extension $\widehat{X}$ of $X$ on the open set $M \cup U$ inclosing $\Im^{+}$, the further extension to $\widehat{M}$ is now trivial, using standard smoothing technology. By continuity, $\mathscr{L}_{\widehat{X}}=\Omega^{-1} X(\Omega) \widehat{g}$ must hold on $\Im^{-}$. This means that the right-hand side smoothly extends there (to zero by hypotheses). In particular, since $\Omega=0$ on $\Im^{-}$, $\widehat{X}(\Omega)=0$ on $\Im^{-}$. That is $\left\langle\widehat{X} \uparrow_{\Im^{-}}, d \Omega\right\rangle=0$, and thus $\widehat{X} \uparrow_{\Im^{-}}$is tangent to $\Im^{-}$as wanted.

The set on $\Im^{-}$of the points where $\widehat{X}$ vanishes is closed since $\widehat{X}$ is continuous. To conclude, we wish to prove that $\widehat{X} \uparrow_{\Im^{-}}$cannot vanish on every (nonempty) open set $A \subset \Im^{-}$(otherwise it vanishes everywhere on $\Im^{-}$, but this case is not allowed by definition of $X$ ). Assume that there is such $A$ where $\widehat{X} \uparrow_{A}=0$, take $p \in A$ and fix any other point $q \in \Im^{-}$, such that there is a $\widehat{g}$-geodesics, $\gamma \subset \Im^{-}$, joining $p$ and $q$. We assume here that $\gamma$ is either a space-like geodesics on $\mathbb{S}^{2}$ or a null-like geodesic at constant angular variables. We want to prove that $\widehat{X}(q)=0$ when $\widehat{X} \uparrow_{A}=0$.

If $\widehat{X} \uparrow_{A}=0$, all the derivatives $\widehat{\nabla}_{a} \widehat{X}^{b}$ vanish, in $A$, when $a \neq \Omega$, that is referring to directions tangent to $\Im^{-}$. However, on $\Im^{-}$it holds $\mathscr{L}_{\widehat{X}} \widehat{g}=0$, by hypotheses. Writing down these equations explicitly, one finds that $\widehat{X}=0$ on $A$ implies $\widehat{\nabla}_{\Omega} \widehat{X}^{b}=0$ if $b \neq \Omega$. However $\widehat{\nabla}_{\Omega} X^{\Omega} \uparrow_{\Im^{-}}=0$ holds since both $X^{\Omega}=X(\Omega)$ and $X(\Omega) / \Omega=X^{\Omega} / \Omega$ vanishes on $\Im^{-}$. We have found that, in $A, \widehat{F}_{a b}=0$. Notice that $\varphi=0$ in $A$, since it is proportional to the limit of $\Omega^{-1} X(\Omega)$ approaching $\Im^{-}$which vanishes by hypotheses. This also entails that $\widehat{K}_{a}=0$ when $a \neq \Omega$, in $A$, that is $\widehat{K}^{a} \neq 0$ for $a=\ell$ at most, in $A$. Let $k$ denote the value $\widehat{K}(p)$ for the considered field $\widehat{X}$ with $\widehat{X} \uparrow_{A}=0$. Let us finally focus on the differential equations 888) referred to the mentioned geodesic $[0,1] \ni t \mapsto \gamma(t)$. We argue that a solution, and thus the unique solution, for initial data at $p, \widehat{X}(0)=0, \widehat{F}_{a b}(0)=0, \widehat{\varphi}(0)=0, \widehat{K}(0):=k$ is $\widehat{X}(t)=0, \widehat{F}_{a b}(t)=0, \widehat{\varphi}(t)=0, \widehat{K}(t)$, for all $t \in[0,1]$, where the last function is the unique satisfying $\dot{\gamma}^{a} \widehat{\nabla}_{a} \widehat{K}_{b}=0$ with $\widehat{K}(0):=k$. To prove it notice that, inserting these functions in (88), the equations reduce to

$$
\dot{\gamma}^{a} \widehat{K}_{a}=0, \quad \dot{\gamma}^{a} \widehat{K}^{b}-\dot{\gamma}^{b} \widehat{K}^{a}=0, \quad \dot{\gamma}^{a} \widehat{\nabla}_{a} \widehat{K}_{b}=0,
$$

The first two equations are certainly fulfilled at $t=0$ by hypotheses, the third one determines $K$ uniquely with the initial condition $\widehat{K}(0):=k$. However also the first two equations are fulfilled on this solution in view of the fact that they are fulfilled at $t=0$ and that $\dot{\gamma}^{a} \widehat{\nabla}_{a} \dot{\gamma}^{b}=0$ since we are dealing with a geodesic. We have found that, in particular, $X$ vanishes at $q$ as wanted, since $X(1)=0$. With the same procedure, moving $p$ and $q$ about the original positions, we find that $X$ vanishes in a open set $A_{q}$ which enlarges $A$ 
and it includes $q$. Iterating the procedure, we can enlarge $A_{q}$ in order to include any third point $q^{\prime} \in \Im^{-}$, joined to $q$ by means of a second geodesics, so that $X$ vanishes at $q^{\prime}$ too. In view of the form (22) of the metric on $\Im^{-}$, for every couple of points $p, q^{\prime} \in \Im^{-}$, there is always a sequence of three consecutive geodesics, of the two above-mentioned types, joining $p$ and $q^{\prime}$. Therefore $X$ vanishes everywhere on $\Im^{-}$. (b) In a neighbourhood of $\Im^{-}$, referring to coordinates $\Omega, \ell, \theta, \phi$ one has

$$
\widehat{X}=f^{\Omega} \partial_{\Omega}+f^{\ell} \partial_{\ell}+f^{\theta} \partial_{\theta}+f^{\phi} \partial_{\phi}
$$

Approaching $\Im^{-}$(i.e. as $\Omega=0$ ) one gets (1) $f^{\Omega}=0$, since $\widehat{X}$ becomes tangent to $\Im^{-}$. However one also finds (2) $\partial_{\Omega} f^{\Omega} \uparrow_{\Im^{-}}=0$ as a consequence of $\left(f^{\Omega}-f^{\Omega} \uparrow_{\Im^{-}}\right) / \Omega=\Omega^{-1} X(\Omega) \rightarrow 0$ approaching $\Im^{-}$. Since $\widehat{X} \uparrow_{\Im^{-}}$ is tangent to the null surface $\Im^{-}$and it is the limit of a timelike vector, we also know that, at the points where it does not vanish, it must be light-like and future directed. Since $\widehat{X}\left\lceil\Im^{-}=f^{\ell} \partial_{\ell}+f^{\theta} \partial_{\theta}+f^{\phi} \partial_{\phi}\right.$, the requirement $\widehat{g}(\widehat{X}, \widehat{X})\left\lceil_{\Im^{-}}=0\right.$ implies that $(3) f^{\theta}=f^{\phi}=0$ everywhere on $\Im^{-}$, in view of the Bondi form of the metric on $\Im^{-}$. Therefore (4) $\widehat{X} \uparrow_{\Im^{-}}=f^{\ell}(0, \ell, \theta, \phi) \partial_{\ell}$. Using Bondi form of the metric again, the requirement $\left(\mathscr{L}_{\widehat{X}} \widehat{g}\right) \uparrow_{\Im^{-}}=0$ produces immediately the constraints $\partial_{\ell} f^{\ell} \uparrow_{\Im^{-}}=0$ in view of (1),(2), (3), and (4), so that $\widehat{X} \uparrow_{\Im^{-}}=f(\theta, \phi) \partial_{\ell}$. Since $\widehat{X} \uparrow_{\Im^{-}}$cannot vanish in any open set on $\Im^{-}, f$ cannot vanish in any open set on $\mathbb{S}^{2}$. Since $f$ is smooth and thus continuous, the set $f^{-1}(0)$ must be closed. Since, with our sign convention for the Bondi metric, both $X$ and $\partial_{\ell}$ are future oriented, $f$ cannot be negative.

Proof of Proposition 3.2. We start from the proofs of (a) and (b). If there were a smooth extension of $Y$ to $\bar{M}=M \cup \Im^{-}$it would be unique by continuity and it would satisfy $\mathscr{L}_{\widehat{Y}} g=0$ up to $\Im^{-}$by continuity again. Therefore it is sufficient to establish the existence of a smooth extension to $\widehat{M}$ to get the most relevant part of (a) and (b). The proof is essentially the same as done in the proof of Proposition 3.1, concerning the existence of the extension of the field $X$. Now, $Y$ is a proper conformal Killing field so that the transport equations (89) Ge77, Hal04 reduces to

$$
\dot{\gamma}^{a} \widehat{\nabla}_{a} \widehat{Y}_{b}=\dot{\gamma}^{a} \widehat{F}_{a b} \quad \text { and } \quad \dot{\gamma}^{a} \widehat{\nabla}_{a} \widehat{F}_{b c}=\widehat{R}_{b c a d} \dot{\gamma}^{a} \widehat{Y}^{d},
$$

The procedure is exactly as that in the proof of Proposition 3.1 and, in this way, one obtains a smooth extention $\widehat{Y}$ of $Y$ on $\widehat{M}$ and in particular on $\Im^{-}$. (c) The condition that $\widehat{Y}$ is tangent to $\Im^{-}$is $\langle\widehat{Y}, d \Omega\rangle=0$ everywhere on $\Im^{-}$. However $g^{s b} \partial_{b} \Omega=\left(\partial_{\ell}\right)^{s}$ and $X \rightarrow f \partial_{\ell}$ approaching $\Im^{-}$, for some nonnegative function $f \in C^{\infty}\left(\mathbb{S}^{2}\right)$, as showed in Proposition 3.1. Therefore $\langle\widehat{Y}, d \Omega\rangle f=\lim _{\rightarrow \Im^{-}} g(\widehat{Y}, X)$. If the limit vanishes approaching $\Im^{+},\langle\widehat{Y}, d \Omega\rangle=0$ on the points $(\ell, s) \in \mathbb{R} \times \mathbb{S}^{2}$ where $f(s) \neq 0$. This happens on an open nonempty set $B \subset \mathbb{S}^{2}$. Therefore $\langle\widehat{Y}, d \Omega\rangle=0$ on $\mathbb{R} \times B$. Let $\left(\ell_{0}, s_{0}\right) \notin \mathbb{R} \times \mathbb{S}^{2}$. Since $\mathbb{S}^{2} \backslash B$ has no interior (see Proposition 3.1), there is a sequence $\mathbb{R} \times B \ni\left(\ell_{0}, s_{n}\right) \rightarrow\left(\ell_{0}, s_{0}\right)$ as $n \rightarrow \infty$. Continuity of $(\ell, s) \mapsto\langle\widehat{Y}, d \Omega\rangle(\ell, s)$ implies $\langle\widehat{Y}, d \Omega\rangle=0$ in $\mathbb{R} \times\left(\mathbb{S}^{2} \backslash B\right)$ and, thus, everywhere. Conversely, if $\widehat{Y}$ is tangent to $\Im^{-}$, then $\langle\widehat{Y}, d \Omega\rangle=0$ on $\Im^{-}$, and hence $\lim _{\rightarrow \Im^{-}} g(\widehat{Y}, X)=\langle\widehat{Y}, d \Omega\rangle f=0$.

To conclude, we prove the last statements: (d) and (e). Since the map $Y \mapsto \widehat{Y} \uparrow_{\varsigma^{-}}$is linear by construction, (e) is a trivial consequence of (d). Let us prove (d). If the considered space is made of the zero vector only, the proof of (d) is trivial. Assume that it is not the case. To demonstrate (d), it is sufficient to prove that the identity $\widehat{Y} \Im_{\Im^{-}}=0$ on a set $A \subset \Im^{+}$which is nonempty and open with respect to the topology of $\Im^{-}$, entails $Y=0$ in $M$ (and thus $\widehat{Y}=0$ in $M \cup \Im^{-}$by continuity). Let us show it. Consider any fixed point $p \in M$ and a smooth path $\gamma$ from some $q \in A$ to $p$ (it exists because $M$ is connected and $\left.\Im^{-}=\partial M\right)$. In view of the first order transport equations (90), $Y(p)=\widehat{Y}(p)=0$ when both $\widehat{Y}(q)$ and $\widehat{F}_{a b}(q)$ vanish. Let us show that it is the case. Suppose that $\left.\widehat{Y}\right|_{\varsigma^{-}}=0$ on $A$ as above. Using coordinates $(\ell, \Omega, \theta, \phi)$ about $\Im^{-}$, one has that $\partial_{a} \widehat{Y}^{b}{ }_{{ }_{A}}=0$ if $a \neq \Omega$. On the other hand, the condition $\mathscr{L}_{\widehat{Y}} \widehat{g}_{a b}=0$ 
computed on $A$, taking into account $\left.\widehat{Y}\right|_{A}=0$ and $\partial_{a} \widehat{Y}^{b} \uparrow_{A}=0$ if $a \neq \Omega$, yields $\partial_{\Omega} \widehat{Y}^{b} \uparrow_{A}=0$, so that $\left.\widehat{\nabla}_{a} \widehat{Y}^{b}\right|_{A}=\partial_{\Omega} \widehat{Y}^{b} \uparrow_{A}+\widehat{\Gamma}_{a c}^{b} \widehat{Y}^{c} \uparrow_{A}=0$. Therefore $\left.F_{a b}\right|_{A}=0$ and it concludes the proof.

Proof of Proposition 3.3 (a) If $\left(s^{1}, s^{2}\right)$ are (local) coordinates of a point $s \in \mathbb{S}^{2}$, fix $\alpha, \beta \in C^{\infty}\left(\mathbb{S}^{2}\right)$ and real constants $r_{1}, r_{2}, r_{3}$. We wish to study the integral lines $t \mapsto(\ell(t), s(t)) \in \mathbb{R} \times \mathbb{S}^{2}$ of the field $Z(\ell, s):=(\alpha(s) \ell+\beta(s)) \partial_{\ell}+\sum_{k=1}^{3} r_{k} S_{k}^{i} \partial_{s^{i}}$ on $\mathbb{R} \times \mathbb{S}^{2}$, with initial condition $\left(\ell_{0}, s_{0}\right)$. By construction, the components referred to the sphere do not depend on $\ell$ and thus, the corresponding equations can be integrated separately. Since $\sum_{k=1}^{3} r_{k} S_{k}^{i} \partial_{s^{i}}$ is smooth and $\mathbb{S}^{2}$ is compact, the integral lines $t \mapsto s\left(t \mid s_{0}\right)$ (here and henceforth $\mid s_{0}$ denotes the initial condition at $t=0$ ) must be smooth and complete (i.e. defined for $t \in(-\infty,+\infty)$ ), in view of well-known theorems of differential equations on manifolds. Then assume that the smooth function $\mathbb{R} \ni t \rightarrow s\left(t \mid s_{0}\right)$ is known (computed as above). The remaining differential equation reads

$$
\frac{d \ell}{d t}=\alpha\left(s\left(t \mid s_{0}\right)\right) \ell+\beta\left(s\left(t \mid s_{0}\right)\right) .
$$

It can be integrated and the right-hand side is defined for the values of $t$ where the full integral ' converges:

$$
\ell\left(t \mid s_{0}, \ell_{0}\right)=e^{\int_{0}^{t} d t_{1} \alpha\left(s\left(t_{1} \mid s_{0}\right)\right)} \ell_{0}+e^{\int_{0}^{t} d t_{1} \alpha\left(s\left(t_{1} \mid s_{0}\right)\right)} \int_{0}^{t} d t_{1} \beta\left(s\left(t_{1} \mid s_{0}\right)\right) e^{-\int_{0}^{t_{1}} d t_{2} \alpha\left(s\left(t_{2} \mid s_{0}\right)\right)} .
$$

It is apparent that the parameter $t$ ranges in the whole real axis due to smoothness of $\mathbb{R} \ni t \rightarrow \alpha\left(s\left(t \mid s_{0}\right)\right)$ and $\mathbb{R} \ni t \rightarrow \beta\left(s\left(t \mid s_{0}\right)\right)$, and that $\mathbb{R} \ni t \mapsto \ell\left(t \mid s_{0}, t_{0}\right)$ is smooth as well. We have established that the integral lines of $Z$ are complete and thus, in view of known theorems, the one-parameter group of diffeomorphisms generated by $Z$ is global. Since $s=s(t)$ must necessarily describe a rotation of $S O(3)$, about the axis $\left(r_{1}, r_{2}, r_{3}\right) / \sqrt{r_{1}^{2}+r_{2}^{2}+r_{3}^{2}}$ with angle $t \sqrt{r_{1}^{2}+r_{2}^{2}+r_{3}^{2}}$, of the point on $\mathbb{S}^{2}$ initially individuated by $s_{0}$ and, taking (91) into account, it is evident that each diffeomorphism

$$
\mathbb{R} \times \mathbb{S}^{2} \ni\left(\ell_{0}, s_{0}\right) \mapsto\left(\ell\left(t \mid s_{0}, t_{0}\right), s\left(t \mid s_{0}\right)\right) \in \mathbb{R} \times \mathbb{S}^{2},
$$

for every fixed $t \in \mathbb{R}$, has the form (26) and, thus, it belongs to $G_{\Im^{-}}$.

(b) A fixed $(a, b, R) \in G_{\Im^{-}}$can be decomposed as

$$
(R, a, b)=\left(I, a \circ R^{-1}, b \circ R^{-1}\right)(R, 0,0) .
$$

Looking at (91), $(R, 0,0)$ is an element of the one-parameter group generated by $\sum_{k=1}^{3} n_{k} S_{k}$, where $\left(n_{1}, n_{2}, n_{3}\right)$ are the Cartesian components of the rotation axis of $R$; conversely the transformation $\left(I, a \circ R^{-1}, b \circ R^{-1}\right)$ can be written as $\exp \{1 Z\}$ where $Z=\ell a\left(R^{-1}(s)\right) \partial_{\ell}+b\left(R^{-1}(s)\right) \partial_{\ell}$.

Proof of Theorem 3.1. Consider the local one-parameter group of diffeomorphisms generated by $\widehat{Y}$ in a sufficiently small neighbourhood (in $\widehat{M}$ ) of a point $q \in \Im^{-}$and for $t \in(-\epsilon, \epsilon)$ with $\epsilon>0$ sufficiently small. In local coordinates over $\Im^{-},\left(\ell, s^{1}, s^{2}\right) \in(a, b) \times A$, such a set of transformations can be represented by

$$
\ell \rightarrow \ell_{t}:=f\left(\ell, s_{1}, s_{2}, t\right), \quad\left(s^{1}, s^{2}\right) \rightarrow\left(s_{t}^{1}, s_{t}^{2}\right):=g\left(\ell, s^{1}, s^{2}, t\right) \quad \text { with }\left(\ell, s^{1}, s^{2}\right) \in(a, b) \times A .
$$

Using the same argument as the one used to characterise the group $G_{\Im^{-}}$(after Proposition 3.2), one finds that it must be $g\left(\ell, s_{1}, s_{2}, t\right)=R_{t}(s)$ for all $\ell, s$ and $f\left(\ell, s_{1}, s_{2}, t\right)=c\left(s_{1}, s_{2}, t\right) \ell+b\left(s_{1}, s_{2}, t\right)$, for all $\ell, s$, for some $R_{t} \in O(3)$ depending on $t$ smoothly, and where $c, b$ are jointly smooth real functions. The requirement, that $t \mapsto R_{t}$ is a (local) one-parameter subgroup of $S O(3)$, implies that $\left.\frac{d R_{t}}{d t}\right|_{t=0}=$ $\sum_{k=1}^{3} r_{k} S_{k}\left(s_{1}, s_{2}\right)$. Similarly $\left.\frac{d f_{t}}{d t}\right|_{t=0}=\left.\frac{\partial c\left(s_{1}, s_{2}, t\right)}{\partial t}\right|_{t=0} \ell+\left.\frac{\partial b\left(s_{1}, s_{2}, t\right)}{\partial t}\right|_{t=0}$. We have found that, in local coordinates

$$
\widehat{Y} \Im_{\Im^{-}}=\sum_{k=1}^{3} r_{k} S_{k}\left(s_{1}, s_{2}\right)+\left.\frac{\partial c\left(s_{1}, s_{2}, t\right)}{\partial t}\right|_{t=0} \ell \partial_{\ell}+\left.\frac{\partial b\left(s_{1}, s_{2}, t\right)}{\partial t}\right|_{t=0} \partial_{\ell},
$$


and thus, about $q, \widehat{Y}\left\lceil_{\Im^{-}}\right.$takes the form of the vectors in $\mathfrak{g}_{\Im^{-}}$. However, since it holds true in a neighbourhood of each point on $\Im^{-}$, we have that $\widehat{Y} \uparrow_{\Im^{-}} \in \mathfrak{g}_{\Im^{-}}$.

To conclude, (b) is an immediate consequence of (a) and of the last part of (a) in Proof of Proposition 3.3 .

Proof of Proposition 3.4. Since $\tilde{Y} \in \mathfrak{g}_{\Im^{-}}$, in principle it has the form

$$
\tilde{Y}(\ell, s)=\sum_{i=1}^{3} c_{i} S_{i}(s)+(f(s)+\ell g(s)) \partial_{\ell} .
$$

Since $\widehat{g}(Y, Y)<0$ about $\Im^{-}$and its limit toward $\Im^{-}$, namely $\widetilde{Y}$, is tangent to $\Im^{-}$it must satisfy $\widehat{g}(\widetilde{Y}, \widetilde{Y})=0$ by continuity (no timelike tangent vectors can be tangent to a null surface). Using the form (22) of $\widehat{g}$ one see that it must be: $\sum_{i=1}^{3} c_{i} S_{i}(s)=0$ on $\Im^{-}$. Using the explicit form of $S_{1}, S_{2}, S_{3}$ referring to the base $\partial_{\phi}, \partial_{\theta}$ of $T \mathbb{S}^{2}$, one sees that this is equivalent to claim that, everywhere on the sphere,

$$
\left(c_{1} \sin \phi-c_{2} \cos \phi\right)=0, \quad c_{1} \cot \theta \cos \phi+c_{2} \cot \theta \sin \phi+c_{3}=0
$$

As a consequence $c_{1}=c_{2}=c_{3}=0$. Therefore, everywhere on $\Im^{-}$

$$
\widetilde{Y}=(f(s)+\ell g(s)) \partial_{\ell},
$$

for some functions $f, g \in C^{\infty}\left(\mathbb{S}^{2}\right)$. $\tilde{Y}$ is the limit of a causal future-directed vector. Therefore, it has either to vanish or to be directed as $\partial_{\ell}$ at every point of $\Im^{-}$. Since $\ell g(s)$ may take every arbitrarily large, positive or negative, value (notice that $g$ is bounded, it being smooth on a compact set), it must be $g(s)=0$ and $f(s) \geq 0$.

\section{B Quasifree states on Weyl algebras}

A $C^{*}$-algebra $\mathcal{W}_{(\mathrm{S}, \sigma)}$ is called Weyl algebra associated with a (real) symplectic space $(\mathrm{S}, \sigma)$ (the symplectic form $\sigma$ being nondegenerate) if it contains a class of non-vanishing elements $W(\psi)$ for all $\psi \in \mathbf{S}$, called Weyl generators, satisfying Weyl relations?:

$$
W(-\psi)=W(\psi)^{*},
$$

$$
W(\psi) W\left(\psi^{\prime}\right)=e^{i \sigma\left(\psi, \psi^{\prime}\right) / 2} W\left(\psi+\psi^{\prime}\right) ;
$$

and $\mathcal{W}_{(\mathrm{S}, \sigma)}$ coincides with the closure of the $*$-algebra (finitely) generated by Weyl generators. $\mathcal{W}_{(\mathrm{S}, \sigma)}$ is uniquely determined by $(\mathrm{S}, \sigma)$ (theorem 5.2.8 in [BR022]): two different realizations admit a unique * isomorphism which transforms the former into the latter preserving Weyl generators and the norm on $\mathcal{W}_{(\mathrm{S}, \sigma)}$ is unique since $*$ isomorphisms of $C^{*}$ algebras are isometric. This result implies that every GNS representation of a Weyl algebra is always faithful and isometric. $\mathcal{W}_{(\mathrm{S}, \sigma)}$ can always be realized in terms of bounded operators on $\ell^{2}(\mathrm{~S})$, viewing $\mathbf{S}$ as a Abelian group and defining the generators as $(W(\psi) F)\left(\psi^{\prime}\right):=$ $e^{-i \sigma\left(\psi, \psi^{\prime}\right) / 2} F\left(\psi+\psi^{\prime}\right)$ for every $F \in \ell^{2}(\mathrm{~S})$. In this realization (and thus in every realization) it is manifest that generators $W(\psi)$ are linearly independent. As a consequence of (W1) and (W2), one gets: $W(0)=\mathbb{I}$ (the unit element), $W(\psi)^{*}=W(\psi)^{-1},\|W(\psi)\|=1$ and, using non degenerateness of $\sigma, W(\psi)=W\left(\psi^{\prime}\right)$ iff $\psi=\psi^{\prime}$. Strong continuity of the unitary group implementing a $*$-automorphism representation $\beta$ of a topological group $G \ni g \mapsto \beta_{g}$ for a $\beta$-invariant state $\omega$ on a Weyl algebra $\mathcal{W}(\mathrm{S}, \sigma)$, is equivalent to

\footnotetext{
${ }^{6}$ Notice that in KW91] a different convention for the sign of $\sigma$ in (W2) is employed.
} 
$\lim _{g \rightarrow \mathbb{I}} \omega\left(W(-\psi) \beta_{g} W(\psi)\right)=1$ for all $\psi \in \mathrm{S}$. The proof follows immediately from $\| \Pi_{\omega}\left(\beta_{g^{\prime}} W(\psi)\right) \Upsilon_{\omega}-$ $\Pi_{\omega}\left(\beta_{g} W(\psi)\right) \Upsilon_{\omega} \|^{2}=2-\omega\left(W(-\psi) \beta_{g^{\prime-1}} W(\psi)\right)-\omega\left(W(-\psi) \beta_{g^{-1} g^{\prime}} W(\psi)\right)$ and $\overline{\Pi_{\omega}(\mathcal{W}(\mathrm{S}, \sigma)) \Upsilon_{\omega}}=\mathfrak{H}_{\omega}$. A state $\omega$ on $\mathcal{W}_{(\mathrm{S}, \sigma)}$, with GNS triple $\left(\mathfrak{H}_{\omega}, \Pi_{\omega}, \Upsilon_{\omega}\right)$, is called regular if the maps $\mathbb{R} \ni t \mapsto \Pi_{\omega}(W(t \psi))$ are strongly continuous. Then, in accordance with Stone theorem, one can write $\Pi_{\omega}(W(\psi))=e^{i \sigma\left(\psi, \Psi_{\omega}\right)}$, $\sigma\left(\psi, \Psi_{\omega}\right)$ being the (self-adjoint) field operator symplectically-smeared with $\psi$. In this way field operators enters the theory in Weyl algebra scenario. Working formally, by Stone theorem (W2) implies $\mathbb{R}$-linearity and standard CCR:

$$
(L) \quad \sigma\left(a \psi+b \psi^{\prime}, \Psi_{\omega}\right)=a \sigma\left(\psi, \Psi_{\omega}\right)+b \sigma\left(\psi^{\prime}, \Psi_{\omega}\right), \quad(C C R) \quad\left[\sigma\left(\psi, \Psi_{\omega}\right), \sigma\left(\psi^{\prime}, \Psi_{\omega}\right)\right]=-i \sigma\left(\psi, \psi^{\prime}\right) I,
$$

for $a, b \in \mathbb{R}$ and $\psi, \psi^{\prime} \in \mathrm{S}$. Actually (L) and (CCR) hold rigorously in an invariant dense set of analytic vectors by Lemma 5.2.12 in BR022 (it holds if $\omega$ is quasifree by proposition B.1 below).

In the standard approach of QFT, based on bosonic real scalar field operators $\Psi$ a, either vector either density matrix, state is quasifree if the associated $n$-point functions (expectation values of a product of $n$ fields) satisfy (i) $\langle\sigma(\psi, \Psi)\rangle=0$ for all $\psi \in \mathrm{S}$ and (ii) the $n$-point functions $\left\langle\sigma\left(\psi_{1}, \Psi\right) \cdots \sigma\left(\psi_{n}, \Psi\right)\right\rangle$ are determined from the functions $\left\langle\sigma\left(\psi_{i}, \Psi\right) \sigma\left(\psi_{j}, \Psi\right)\right\rangle$, with $i, j=1,2, \cdots, n$, using standard Wick's expansion. A technically different but substantially equivalent definition, completely based on the Weyl algebra was presented in KW91. It relies on the following three observations: (a) Working formally with (i) and (ii), one finds that it holds $\left\langle e^{i \sigma(\psi, \Psi)}\right\rangle=e^{-\langle\sigma(\psi, \Psi) \sigma(\psi, \Psi)\rangle / 2}$. In turn, at least formally, such identity determines the $n$-point functions (reproducing Wick's rule) by Stone theorem and (W2). (b) From (CCR) it holds $\left\langle\sigma(\psi, \Psi) \sigma\left(\psi^{\prime}, \Psi\right)\right\rangle=\mu\left(\psi, \psi^{\prime}\right)-(i / 2) \sigma\left(\psi, \psi^{\prime}\right)$, where $\mu\left(\psi, \psi^{\prime}\right)$ is the symmetrised two-point function $(1 / 2)\left(\left\langle\sigma(\psi, \Psi) \sigma\left(\psi^{\prime}, \Psi\right)\right\rangle+\left\langle\sigma\left(\psi^{\prime}, \Psi\right) \sigma(\psi, \Psi)\right\rangle\right)$ which defines a symmetric positive-semidefined bilinear form on S. (c) $\left\langle A^{\dagger} A\right\rangle \geq 0$ for elements $A:=\left[e^{i \sigma(\psi, \Psi)}-I\right]+i\left[e^{i \sigma(\psi, \Psi)}-I\right]$ entails:

$$
\left|\sigma\left(\psi, \psi^{\prime}\right)\right|^{2} \leq 4 \mu(\psi, \psi) \mu\left(\psi^{\prime}, \psi^{\prime}\right), \quad \text { for every } \psi, \psi^{\prime} \in \mathbf{S},
$$

which, in turn, implies that $\mu$ is strictly positive defined because $\sigma$ is non degenerate. Reversing the procedure, the general definition of quasifree states on Weyl algebras is the following.

Definition B.1. Let $\mathcal{W}_{\mathrm{S}, \sigma}$ be a Weyl algebra and $\mu$ a real scalar product on $\mathrm{S}$ satisfying (93). A state $\omega_{\mu}$ on $\mathcal{W}_{\mathrm{S}, \sigma}$ is called a quasifree state associated with $\mu$ if

$$
\omega_{\mu}(W(\psi)):=e^{-\mu(\psi, \psi) / 2}, \quad \text { for all } \psi \in \mathrm{S} .
$$

Lemma B.1. Let $(\mathrm{S}, \sigma)$ be a real symplectic space with $\sigma$ non degenerate and $\mu$ a real scalar product on $\mathrm{S}$ satisfying (93). There is a complex Hilbert space $\mathcal{H}_{\mu}$ and a map $K_{\mu}: \mathrm{S} \rightarrow \mathcal{H}_{\mu}$ with:

(i) $K_{\mu}$ is $\mathbb{R}$-linear with dense complexified range, i.e. $\overline{K_{\mu}(\mathrm{S})+i K_{\mu}(\mathrm{S})}=\mathcal{H}_{\mu}$,

(ii) for all $\psi, \psi^{\prime} \in \mathrm{S},\left\langle K_{\mu} \psi, K_{\mu} \psi^{\prime}\right\rangle=\mu\left(\psi, \psi^{\prime}\right)-(i / 2) \sigma\left(\psi, \psi^{\prime}\right)$.

Conversely, if the pair $(\mathcal{H}, K)$ satisfies $(i)$ and $\sigma\left(\psi, \psi^{\prime}\right)=-2 \operatorname{Im}\left\langle K \psi, K \psi^{\prime}\right\rangle_{\mathcal{H}}$, with $\psi, \psi^{\prime} \in \mathrm{S}$, the unique real scalar product $\mu$ on $\mathrm{S}$ satisfying (ii) verifies (93).

The last statement arises by Cauchy-Schwarz inequality, the remaining part being in Proposition 3.1 in KW91. Notice that $K_{\mu}$ is always injective due either to (ii) either to non degenerateness of $\sigma$. Now existence of quasifree states can be proved using the above lemma with the following proposition. Therein, uniqueness and regularity of the state is contained in Lemma A.2 and Proposition 3.1 in [KW91].

Proposition B.1. For every $\mu$ as in definition B.1 the following hold.

(a) there is a unique quasifree state $\omega_{\mu}$ associated with $\mu$ and it is regular. 
(b) The GNS triple $\left(\mathfrak{H}_{\omega_{\mu}}, \Pi_{\omega_{\mu}}, \Upsilon_{\omega_{\mu}}\right)$ is determined as follows with respect to $\left(\mathcal{H}_{\mu}, K_{\mu}\right)$ in lemma (B.1). (i) $\mathfrak{H}_{\omega_{\mu}}$ is the symmetric Fock space with one-particle space $\mathcal{H}_{\mu}$. (ii) The cyclic vector $\Upsilon_{\omega_{\mu}}$ is the vacuum vector of $\mathfrak{H}_{\omega}$. (iii) $\Pi_{\omega_{\mu}}$ is determined by $\Pi_{\omega_{\mu}}(W(\psi))=e^{\overline{i(\psi, \Psi)}}$, the bar denoting the closure, wher 7

$$
\sigma(\psi, \Psi):=i a\left(K_{\mu} \psi\right)-i a^{\dagger}\left(K_{\mu} \psi\right), \quad \text { for all } \psi \in \mathrm{S}
$$

$a(\phi)$ and $a^{\dagger}(\phi), \phi \in \mathcal{H}_{\mu}$, being the usual annihilation (antilinear in $\phi$ ) and creation operators defined in the dense linear manifold spanned by the states with finite number of particles.

(c) A pair $(\mathcal{H}, K) \neq\left(\mathcal{H}_{\mu}, K_{\mu}\right)$ satisfies (i) and (ii) in lemma B.1 for $\mu$, determining the same quasifree state $\omega_{\mu}$, if and only if there is a unitary operator $U: \mathcal{H}_{\mu} \rightarrow \mathcal{H}$ such that $U K_{\mu}=K$.

(d) $\omega_{\mu}$ is pure (i.e. its GNS representation is irreducible) if and only if $\overline{K_{\mu}(\mathrm{S})}=\mathcal{H}_{\mu}, 8$.

\section{References}

[AGM00] O. Aharony, S. S. Gubser, J. M. Maldacena, H. Ooguri and Y. Oz, "Large N field theories, string theory and gravity", Phys. Rept. 323 (2000) 183 arXiv:hep-th/9905111].

[Al85] B. Allen, "Vacuum states in the Sitter space", Phys. Rew. D 32 (1985) 3136.

[Ar99] H. Araki: "Mathematical Theory of Quantum Fields", Oxford University Press (1999).

[AX78] A. Ashtekar and B. C. Xanthopoulos, "Isometries compatible with asymptotic flatness at null infinity: a complete description", J. Math. Phys. 19 (1978) 2216.

[BGP96] C. Bär, N. Ginoux, F. Pfäffle, "Wave equations on Lorentzian manifolds and quantization", ESI Lectures in Mathematics and Physics, European Mathematical Society Publishing House (2007).

[BD82] N.D. Birrel and P.C.W. Davies, "Quantum Field Theory in Curved Space", Cambridge University Press (1982).

[BMG94] J. Bros, U. Moschella and J. P. Gazeau, "Quantum field theory in the de Sitter universe", Phys. Rev. Lett. 73 (1994) 1746.

[BM96] J. Bros and U. Moschella, "Two-point Functions and Quantum Fields in de Sitter Universe," Rev. Math. Phys. 8 (1996) 327 arXiv:gr-qc/9511019].

[BD78] T.S. Bunch and P.C.W. Davies, "Quantum Fields theory in de Sitter space: renoramlization by point-splitting", Proc. R. Soc. Lond. A 360 (1978) 117.

[BR021] O. Bratteli, D. W. Robinson, "Operator Algebras And Quantum Statistical Mechanics. Vol. 1: $C^{*}$ And $W^{*}$ Algebras, Symmetry Groups, Decomposition Of States", second edition, Springer-Verlag (2002).

[BR022] O. Bratteli, D. W. Robinson, "Operator algebras and quantum statistical mechanics. Vol. 2: Equilibrium states. Models in quantum statistical mechanics", second edition, Springer (2002).

\footnotetext{
${ }^{7}$ The field operator $\Phi(f)$, with $f$ in the complex Hilbert space $\mathfrak{h}$, used in [BR022] in propositions 5.2.3 and 5.2.4 is related to $\sigma(\psi, \Psi)$ by means of $\sigma(\psi, \Psi)=\sqrt{2} \Phi\left(i K_{\mu} \psi\right)$ assuming $\mathcal{H}:=\mathfrak{h}$.

${ }^{8}$ In turn this is equivalent (see p.77 in KW91]) to $4 \mu\left(\psi^{\prime}, \psi^{\prime}\right)=\sup _{\psi \in \mathrm{S} \backslash\{0\}}\left|\sigma\left(\psi, \psi^{\prime}\right)\right| / \mu(\psi, \psi)$ for every $\psi^{\prime} \in \mathrm{S}$.
} 
[DMP06] C. Dappiaggi, V. Moretti and N. Pinamonti, "Rigorous steps towards holography in asymptotically flat spacetimes", Rev. Math. Phys. 18 (2006) 349.

[Da07] C. Dappiaggi, "Projecting massive scalar fields to null infinity", arXiv:0705.0284] [gr-qc], to appear on Ann. Henri Poincaré.

[Di80] J. Dimock: "Algebras of Local Observables on a Manifold", Commun. Math. Phys. 77 (1980), 219.

[DR02] M. Duetsch and K. H. Rehren, "Generalized free fields and the AdS-CFT correspondence", Annales Henri Poincare 4 (2003) 613 arXiv:math-ph/0209035.

[GR95] I. S. Gradshteyn, I. M. Ryzhik, Table of Integrals, Series, and Products, Fifth Edition, Academic Press, (1995).

[Ge77] R. Geroch in: P. Esposito, L. Witten (Eds.) "Asymptotic Structure of Spacetime", Plenum (1977).

[Ge77] R. Geroch, "Limits of Spacetimes", Commun. Math. Phys 13, 180-193 (1969).

[Haa92] R. Haag, "Local quantum physics: Fields, particles, algebras", Second Revised and Enlarged Edition, Springer (1992).

[Hal04] G.S. Hall "Symmetries and Curvature Structire in General Relativity", World Scientific Publishing, (2004).

[Ho00] S. Hollands: "Aspects of Quantum Field Theory in Curved Spacetime", Ph.D. Thesis - University of York (2000), advisor B.S. Kay, unpublished.

[HW05] S. Hollands, R.M. Wald: "Conservation of the stress tensor in interacting quantum field theory in curved spacetimes". Rev. Math. Phys. 17, (2005) 227. arXiv:gr-qc/0404074

[Is04] J. N. Islam: "An introduction to mathematical cosmology", Cambridge Univ. Press., Cambridge (2004).

[JS02] W. Junker and E. Schrohe, "Adiabatic vacuum states on general spacetime manifolds: Definition, construction, and physical properties", Annales Poincare Phys. Theor. 3 (2002) 1113 arXiv:math-ph/0109010.

[Ka78] B.S. Kay, "Linear Spin-Zero Quantum Fields in External Gravitational and Scalar Fields", Commun. Math. Phys 62, (1978) 55.

[KW91] B. S. Kay, R. M. Wald, "Theorems On The Uniqueness And Thermal Properties Of Stationary, Nonsingular, Quasifree States On Space-Times With A Bifurcate Killing Horizon", Phys. Rept. 207, 49 (1991).

[Le53] J. Leray, "Hyperbolic Differential Equations", Unpublished Lecture Notes, Princeton (1953).

[Li96] A. Linde: "Particle Physics and Inflationary Cosmology", Harwood Academic Publishers (1996).

[LR90] C. Lüders and J. E. Roberts, "Local Quasiequivalence and Adiabatic Vacuum States", Comm. Math. Phys. 134, (1990) 29.

[Mo06] V. Moretti, "Uniqueness theorem for BMS-invariant states of scalar QFT on the null boundary of asymptotically flat spacetimes and bulk-boundary observable", Commun. Math. Phys. 268, (2006) 726 arXiv:gr-qc/0512049. 
[Mo07] V. Moretti, "Quantum out-states states holographically induced by asymptotic flatness: Invariance under spacetime symmetries, energy positivity and Hadamard property", arXiv:gr-qc/0610143 to appear on Comm. Math. Phys.

[Mo03] V. Moretti, "Comments on the stress energy tensor operator in curved space-time". Commun. Math. Phys. 232, (2003) 189 arXiv:gr-qc/0109048].

[Pi05] N. Pinamonti, "De Sitter quantum scalar field and horizon holography", arXiv: hep-th/0505179.

[Ol07] H. Olbermann, "States of low energy on Robertson-Walker spacetimes", Class. Quantum. Grav. 24, (2007) 5011 arXiv:0704.2986 [gr-qc]].

[Pa69] L. Parker, "Quantized fields and particle creation in expanding universes. 1", Phys. Rev. 183, (1969) 1057 .

[Ri06] W. Rindler, "Relativity. Special, General and Cosmological", Second Edition, Oxford University Press, (2006).

[SS76] C. Schomblond and P. Spindel "Conditions d'unicité pour le propagateur $\Delta^{(1)}(x, y)$ du champ scalaire dans l'univers de de Sitter", Ann. Inst. Henri Poincaré 25, (1976) 67.

[Wa84] R. M. Wald: “General Relativity”, Chicago University Press, (1984).

[Wa94] R. M. Wald: "Quantum field theory in curved space-time and black hole thermodynamics", The University of Chicago Press (1994). 